\title{
Total cross sections in five methods for two-electron capture by alpha particles from helium: CDW-4B, BDW-4B, BCIS-4B, CDW-EIS-4B and CB1-4B
}

\section{Dževad Belkić ${ }^{1,2}$}

Received: 6 February 2020 / Accepted: 18 March 2020 / Published online: 17 April 2020 (c) The Author(s) 2020

\begin{abstract}
This work is on quantum-mechanical four-body distorted wave theories for double electron capture in collisions between fast heavy multiply charged ions and heliumlike atomic systems. The five widely used distorted wave methods of the first- and second-order in the perturbation series expansions are compared with the available experimental data on $\alpha-\mathrm{He}$ collisions. These are the four-body boundary-corrected first Born (CB1-4B), the boundary-corrected continuum intermediate state (BCIS$4 \mathrm{~B})$, the Born distorted wave (BDW-4B), the continuum distorted wave (CDW-4B) and the continuum distorted wave-eikonal initial state (CDW-EIS-4B) methods. We address the complete breakdown of the CDW-EIS-4B method at all impact energies within its expected validity domain (100-10000 keV). Further, the relative performance is evaluated of the second-order theories with and without the eikonalization of the two-electron Coulomb wavefunctions for double continuum intermediate states. Finally, at all the considered intermediate and high energies, the practical aspects of the studied five methods are investigated by protracted evaluations of the convergence rates of total cross sections as a function of the number of quadrature points per axis in numerical computations of multi-dimensional (3D-5D) integrals.
\end{abstract}

Keywords Double charge exchange $\cdot$ Distorted wave methods $\cdot$ Rearranging collisions · Two-electron continuum states

Dževad Belkić

Dzevad.Belkic@ki.se

1 Department of Oncology and Pathology, Karolinska Institute, P.O. Box 260, 17176 Stockholm, Sweden

2 Radiation Physics and Nuclear Medicine, Karolinska University Hospital, P.O. Box 260, 17176 Stockholm, Sweden 


\section{Introduction}

Over the years, an emphasis has been given to the electronic eikonal initial states (EIS). These employ the well-known asymptotic phase factor of the single-electron Coulomb wavefunction in the entrance channel. Here, the word "asymptotic" refers to infinitely large distances between the active target electron and the projectile nucleus.

By contrast, the electronic full Coulomb wavefunctions are used in the continuum distorted wave (CDW) method for high-energy ion-atom collisions [1,2]. This is a well-established method which has extensively been reviewed during the past several decades [3-9]. Its simplified version is called the continuum distorted wave-eikonal initial state (CDW-EIS) approximation [10]. The CDW-EIS method differs from the CDW method only in the replacement of the electronic full Coulomb wavefunction in the entrance channel by its asymptotic behavior (the logarithmic Coulomb phase).

The apparent success of this eikonalization cannot be explained on a sound theoretical basis. Normalization of the initial total scattering wavefunction was initially invoked as an attempt to somehow justify this electronic eikonalization [10]. However, this is not convincing nor consistent, since the CDW-EIS method employs the unnormalized final total scattering states of the CDW method. If indeed the normalized scattering states were the main motivation for eikonalization, then the initial and final electronic full Coulomb wavefunctions for the continuum intermediate states ought to be treated symmetrically on the same footing and, hence, both should be eikonalized.

This has not been done since it would result in downgrading the CDW-EIS method from its second-order status to a first-order perturbation formalism. The latter treatment is known as the symmetric eikonal (SE) method [11]. Moreover, if normalization of total scattering states were to be blamed for the alleged inadequacy of the CDW method with decreasing impact energies, then its surrogate, i.e. the CDW-EIS method, should be successful also for double charge exchange, similarly to single electron capture. Alas, such hopes proved to be in vain $[6,8]$. Total cross sections in the CDW-EIS method for double charge exchange have been found to be several orders of magnitude smaller than the available experimental data!

This happened at intermediate-to-moderately-high impact energies where the CDW-EIS method is supposed to be fully applicable. Such a demise of the two-electron eikonalization marks a sharp breakdown of the CDW-EIS method for double charge exchange. The probability of event occurrence is much weaker for double- than for single-electron capture. This implies an increased sensitivity (of double- compared with single-electron transfer) to the theoretical assumptions of the same type. As such, within the CDW-EIS method, what used to be a reasonable assumption for single charge exchange (one electronic eikonalization), turned out to flagrantly fail for double charge exchange (two electronic eikonalizations). Overall, the literature has witnessed the rise (three-body) and the fall (four-body) of the CDW-EIS method for one- and two-electron transfer, respectively.

For book-keeping, it is important to emphasize that the electronic eikonalization in the CDW-EIS method deals with asymptotically large electron-nucleus separations. Of course, a full Coulomb wavefunction of an electron in the electrostatic field of a nucleus can be replaced by its logarithmic phase, but only at very large electron-nucleus distances. However, such a replacement should not be made in the transition ampli- 
tude because the invoked integrals therein cover all distances. Moreover, due to the exponentially decreasing initial and final bound-state wavefunctions, small electronnucleus distances, in fact, yield the major contributions to the integrals in the T-matrix elements. It is precisely this latter spatial region which is chiefly responsible for the sharply different contributions (to the transition amplitudes) from the full Coulomb wave and its asymptotic phase.

The present comprehensive computations shed some new light onto these intricacies of the perturbative distorted wave formalism of ion-atom scattering theory with four actively participating particles. In the illustrations, we focus on prototype symmetric collisions with two-electron capture by alpha particles from helium targets. Particularly for this resonant collision, it has previously been shown, in e.g. the CDW-4B method, that the combined final singly- and doubly-excited states yield a small contribution. Therefore, it suffices to consider only the transition from the initial to the final ground states, as shall be done in this study. The literature on double charge exchange in ionatom collisions is abundant both with theories [4-8, 12-62] and experiments [63-94].

An obvious drawback of the CDW-EIS method is the loss of symmetry by treating the incident and target nuclear charge on an unequal footing. When one is willing to sacrifice this symmetry, then the possibility opens up for the introduction of some other hybrid first- and second-order approximations with an alternative eikonalization of Coulomb continuum intermediate states. This alternative deals with eikonalization of the relative motion of heavy nuclei in lieu of the electronic eikonalization from the CDW-EIS method.

From a theoretical viewpoint, it is by far more justified to perform the eikonalization of Coulomb wavefunctions for inter-nuclear than that for electron-nucleus interactions. This is exclusively due to the large reduced mass $\mu$ of two heavy nuclei. It is wellknown that the replacement of the full Coulomb wavefunction for the inter-nuclear potential by its eikonal logarithmic phase factor gives a negligible $1 / \mu^{2}$ contribution to the total cross section. This has been shown for the full (exact) eikonal T-matrix element [3]. Therefore, the same conclusion ought to hold true also for all the ensuing specific approximations, extracted from the full eikonal transition amplitude either perturbatively (to any given order) or non-perturbatively [20]. The said replacement is amply justified even much below the Massey resonance peak (i.e. notably below $25 \mathrm{keV} / \mathrm{amu}$ ) and, most importantly, without the need to resort to any large, asymptotic distance between the two nuclei. This heavy particle eikonalization should be contrasted to the CDW-EIS method where the electronic eikonalization is applicable only to asymptotically large electron-nucleus separation.

Such a theoretical argument suffices to anticipate that the hybrid second-order methods based upon the eikonalized Coulomb wavefunctions for the relative motion of heavy nuclei should exhibit a reasonably successful performance for one- and double-electron capture. These are the boundary-corrected continuum intermediate state (BCIS) [8, 41] and the Born distorted wave (BDW) [49, 50] methods. Such a stratification leads to yet another level of the present testings by confronting the fourbody versions of the CDW-EIS and BCIS or BDW methods for which their three-body variants are known to perform with a comparable adequacy relative to measurements. Our choice of double capture is an excellent candidate for this type of testing aimed 
at determining which of the two mentioned eikonalizations for electronic or nuclear motions is more successful with respect to the corresponding experimental data.

Additionally, for the total cross sections in the CB1-4B, BCIS-4B, BDW-4B and CDW-4B methods, we carry out a detailed examination (tabular, graphical) of the convergence rates of the numerically computed multiple-dimensional (3D-5D) integrals as a function of the Gauss-Legendre points per each axis of the invoked quadratures.

Atomic units will be used throughout unless otherwise stated.

\section{Theory}

We begin by recapitulating the basic features of the kinematics and dynamics for double charge exchange in the four-body distorted wave formalism. First, it should be emphasized that one of the critically important problems for testing theories in fourparticle ion-atom collisions is double charge exchange (or double electron transfer or double electron capture). Here, two electrons $e_{1}$ and $e_{2}$, that are initially bound to the target nucleus (T), both end up finally in another bound state, but this time around the projectile nucleus $(\mathrm{P})$. This process is symbolized by:

$$
Z_{\mathrm{P}}+\left(Z_{\mathrm{T}} ; e_{1}, e_{2}\right)_{i} \longrightarrow\left(Z_{\mathrm{P}} ; e_{1}, e_{2}\right)_{f}+Z_{\mathrm{T}},
$$

or equivalently,

$$
\mathrm{P}+\left(\mathrm{T} ; e_{1}, e_{2}\right)_{i} \longrightarrow\left(\mathrm{P} ; e_{1}, e_{2}\right)_{f}+\mathrm{T}
$$

where the parentheses represent the bound states, whereas $Z_{\mathrm{P}}$ and $Z_{\mathrm{T}}$ are the nuclear charges of $\mathrm{P}$ and $\mathrm{T}$, respectively. The indices $i$ and $f$ denote the sets of the usual quantum numbers of the initial and final bound states, respectively.

Let $\boldsymbol{x}_{j}$ and $\boldsymbol{s}_{j}$ be the position vectors of $e_{j}$ relative to T and P, respectively ( $j=$ $1,2)$. Further, let $R$ be the internuclear axis with $\boldsymbol{R}$ being the position vector of $\mathrm{P}$ relative to T. We denote by $\boldsymbol{r}_{i}$ and $\boldsymbol{r}_{f}$ the position vectors of $\mathrm{P}$ and $\mathrm{T}$ relative to the center-of-mass of $\left(Z_{\mathrm{T}} ; e_{1}, e_{2}\right)_{i}$ and $\left(Z_{\mathrm{P}} ; e_{1}, e_{2}\right)_{f}$, respectively. The elements of the set $\left\{\boldsymbol{r}_{i}, \boldsymbol{r}_{f}, \boldsymbol{x}_{1,2}, \boldsymbol{s}_{1,2}\right\}$ can be connected to each other by introducing the position vectors $\left\{\boldsymbol{r}_{\mathrm{P}}, \boldsymbol{r}_{\mathrm{T}}, \boldsymbol{r}_{e_{1,2}}\right\}$ of $\left\{\mathrm{P}, \mathrm{T}, e_{1,2}\right\}$ relative to the origin $\mathrm{O}$ of an arbitrary Galilean reference frame XOYZ.

Such a setting gives the defining expressions for the vectors $\left\{\boldsymbol{x}_{1,2}, \boldsymbol{s}_{1,2}, \boldsymbol{r}_{i}, \boldsymbol{r}_{f}\right\}$ as well as for the vector $\boldsymbol{R}$ of the inter-nuclear separation $R$ and the vector $\boldsymbol{r}_{12}$ of the inter-electronic distance $r_{12}$ :

$$
\begin{aligned}
\boldsymbol{x}_{1,2} & =\boldsymbol{r}_{e_{1,2}}-\boldsymbol{r}_{\mathrm{T}}, \boldsymbol{s}_{1,2}=\boldsymbol{r}_{e_{1,2}}-\boldsymbol{r}_{\mathrm{P}}, \\
\boldsymbol{R} & =\boldsymbol{r}_{\mathrm{P}}-\boldsymbol{r}_{\mathrm{T}}, \boldsymbol{r}_{12}=\boldsymbol{r}_{e_{1}}-\boldsymbol{r}_{e_{2}}, \\
\boldsymbol{r}_{i} & =\boldsymbol{r}_{\mathrm{P}}-\frac{M_{\mathrm{T}} \boldsymbol{r}_{\mathrm{T}}+\boldsymbol{r}_{e_{1}}+\boldsymbol{r}_{e_{2}}}{M_{\mathrm{T}}+2}, \boldsymbol{r}_{f}=\boldsymbol{r}_{\mathrm{T}}-\frac{M_{\mathrm{P}} \boldsymbol{r}_{\mathrm{P}}+\boldsymbol{r}_{e_{1}}+\boldsymbol{r}_{e_{2}}}{M_{\mathrm{P}}+2},
\end{aligned}
$$


where $M_{\mathrm{J}}$ is the mass of the Kth nucleus $(\mathrm{J}=\mathrm{P}, \mathrm{T})$. Here, the electron mass $m_{e}$ does not explicitly appear since $m_{e}=1$ in the adopted atomic units. The sought inter-relations of the introduced vectors read as:

$$
\begin{aligned}
\boldsymbol{R} & =\boldsymbol{x}_{1}-\boldsymbol{s}_{1}=\boldsymbol{x}_{2}-\boldsymbol{s}_{2} \\
\boldsymbol{x}_{12} & =\boldsymbol{x}_{1}-\boldsymbol{x}_{2}, \boldsymbol{s}_{12}=\boldsymbol{s}_{1}-\boldsymbol{s}_{2}, \boldsymbol{x}_{12}=\boldsymbol{s}_{12} \equiv \boldsymbol{r}_{12} \\
\boldsymbol{r}_{i} & =m_{i} \boldsymbol{x}_{1}-\boldsymbol{s}_{1}, \boldsymbol{r}_{i}=-m_{f} \boldsymbol{r}_{f}+\frac{m_{f}}{\mu_{f}} \boldsymbol{s}_{1} \\
\boldsymbol{r}_{f} & =m_{f} \boldsymbol{s}_{1}-\boldsymbol{x}_{1}, \boldsymbol{r}_{f}=-m_{i} \boldsymbol{r}_{i}-\frac{m_{i}}{\mu_{i}} \boldsymbol{x}_{1}
\end{aligned}
$$

where $m_{i}$ or $m_{f}$ is the reduced mass of $(\mathrm{T}, e)_{i}$ or $(\mathrm{P}, e)_{f}$, and $\mu_{i}$ or $\mu_{f}$ is the reduced mass of $\mathrm{P}+\left(\mathrm{T} ; e_{1}, e_{2}\right)_{i}$ or $\left(\mathrm{P} ; e_{1}, e_{2}\right)_{f}+\mathrm{T}$, respectively:

$$
\begin{gathered}
m_{i}=\frac{M_{\mathrm{T}}}{M_{\mathrm{T}}+1}, \quad \mu_{i}=\frac{M_{\mathrm{P}}\left(M_{\mathrm{T}}+2\right)}{M_{\mathrm{P}}+M_{\mathrm{T}}+2}, \\
m_{f}=\frac{M_{\mathrm{P}}}{M_{\mathrm{P}}+1}, \quad \mu_{f}=\frac{M_{\mathrm{T}}\left(M_{\mathrm{P}}+2\right)}{M_{\mathrm{T}}+M_{\mathrm{P}}+2} .
\end{gathered}
$$

The Hamiltonians in the entrance and exit channels are given by the following formulae in the center-of-mass (c.m.) system:

$$
\begin{aligned}
& H_{i}=K_{i}+h_{i}, \quad K_{i}=-\frac{1}{\mu_{i}} \nabla_{\boldsymbol{r}_{i}}^{2} \\
& h_{i}=-\frac{1}{m_{i}} \nabla_{\boldsymbol{x}_{1}}^{2}-\frac{1}{m_{i}} \nabla_{\boldsymbol{x}_{2}}^{2}-\frac{1}{M_{\mathrm{T}}} \nabla_{\boldsymbol{x}_{1}} \cdot \nabla_{\boldsymbol{x}_{2}}+V_{\mathrm{T}}, \\
& H_{f}=K_{f}+h_{f}, K_{f}=-\frac{1}{\mu_{f}} \nabla_{\boldsymbol{r}_{f}}^{2} \\
& h_{f}=-\frac{1}{m_{f}} \nabla_{\boldsymbol{s}_{1}}^{2}-\frac{1}{m_{f}} \nabla_{\boldsymbol{s}_{2}}^{2}-\frac{1}{M_{\mathrm{P}}} \nabla_{\boldsymbol{s}_{1}} \cdot \nabla_{\boldsymbol{s}_{2}}+V_{\mathrm{P}},
\end{aligned}
$$

where $V_{\mathrm{T}}$ and $V_{\mathrm{P}}$ are the full interactions in the bound-state heliumlike systems $\left(\mathrm{T} ; e_{1}, e_{2}\right)_{i}$ and $\left(\mathrm{P} ; e_{1}, e_{2}\right)_{f}$ :

$$
\begin{aligned}
& V_{\mathrm{T}}=V_{\mathrm{T} e_{1}}+V_{\mathrm{T} e_{2}}+V_{12}, \quad V_{\mathrm{P}}=V_{\mathrm{P} e_{1}}+V_{\mathrm{P} e_{2}}+V_{12} \\
& V_{\mathrm{T} e_{1,2}}=-\frac{Z_{\mathrm{T}}}{x_{1,2}}, \quad V_{\mathrm{P} e_{1,2}}=-\frac{Z_{\mathrm{P}}}{s_{1,2}}, \quad V_{e_{1} e_{2}} \equiv V_{12}=\frac{1}{x_{12}} .
\end{aligned}
$$

In (2.6), $\boldsymbol{k}_{i}$ and $\boldsymbol{k}_{f}$ are the initial and final wave vectors defined by $\boldsymbol{k}_{i}=\mu_{i} \boldsymbol{v}_{i}$ and $\boldsymbol{k}_{f}=\mu_{f} \boldsymbol{v}_{f}$ where $\boldsymbol{v}_{i}$ and $\boldsymbol{v}_{f}$ are the velocities of the impact and scattered projectile, respectively. Following the standard convention, the initial wave vector $\boldsymbol{k}_{i}$ represents the momentum of $\mathrm{P}$ with respect to $\left(\mathrm{T} ; e_{1}, e_{2}\right)_{i}$. However, precisely opposed to the standard convention, the final wave vector $\boldsymbol{k}_{f}$ is presently defined as the momentum of $\left(\mathrm{P} ; e_{1}, e_{2}\right)_{f}$ with respect to $\mathrm{T}$. 
This is done in order that $\boldsymbol{k}_{f}$ satisfies the relation $\boldsymbol{k}_{f}=\mu_{f} \boldsymbol{v}_{f}$, which is symmetrical to $\boldsymbol{k}_{i}=\mu_{i} \boldsymbol{v}_{i}$. Such a kinematic similarity between $\boldsymbol{k}_{i}$ and $\boldsymbol{k}_{f}$ becomes particularly useful for four-body problems with two heavy nuclei $\left(M_{\mathrm{J}} \gg 1 ; \mathrm{J}=\mathrm{P}, \mathrm{T}\right)$ and two electrons where the eikonal approximation is appropriate, in which case $\hat{\boldsymbol{k}}_{f} \approx \hat{\boldsymbol{k}}_{i}$, where $\hat{\boldsymbol{k}}_{j}=\boldsymbol{k}_{j} / k_{j}(j=i, f)$. In (2.6), $K_{j}$ and $h_{j}(j=i, f)$ are the kinetic energy operator of the relative motion of the two heavy scattering aggregates and the electronic Hamiltonian for the electrons-nucleus states, respectively.

The dynamic inter-electron coupling terms due to gradient-gradient potential operators $-\left(1 / M_{\mathrm{T}}\right) \boldsymbol{\nabla}_{\boldsymbol{x}_{1}} \cdot \boldsymbol{\nabla}_{\boldsymbol{x}_{2}}$ and $-\left(1 / M_{\mathrm{P}}\right) \boldsymbol{\nabla}_{\boldsymbol{s}_{1}} \cdot \boldsymbol{\nabla}_{\boldsymbol{s}_{2}}$ from $h_{i}$ and $h_{f}$ are too weak to be retained for the kinetics in process $(2.1)$ because they are heavily damped by the small factors $1 / M_{\mathrm{T}}$ and $1 / M_{\mathrm{P}}$. Therefore, these electronic couplings, that are also known as the mass-polarization terms, can safely be neglected in the case of heavy masses of nuclei $\mathrm{T}$ and $\mathrm{P}$, as typical for the eikonal approximation. Consequently, the total Hamiltonian $H$ of the whole system $\mathrm{P}+\left(\mathrm{T} ; e_{1}, e_{2}\right)_{i}$ or $\left(\mathrm{P} ; e_{1}, e_{2}\right)_{f}+\mathrm{T}$ in the c.m. reference frame is given by:

$$
H=H_{i}+V_{i}=H_{f}+V_{f}
$$

where $V_{i}$ and $V_{f}$ are the perturbations in the entrance and exit channel, respectively:

$$
V_{i}=V_{\mathrm{P}}+V_{\mathrm{PT}}, \quad V_{f}=V_{\mathrm{T}}+V_{\mathrm{PT}}, \quad V_{\mathrm{PT}}=\frac{Z_{\mathrm{P}} Z_{\mathrm{T}}}{R} .
$$

In the asymptotic region of large inter-aggregate separations, perturbations $V_{i}$ and $V_{f}$ reduce to pure Coulomb potentials:

$$
V_{i}^{\infty}=\frac{Z_{\mathrm{P}}\left(Z_{\mathrm{T}}-2\right)}{R}, V_{f}^{\infty}=\frac{Z_{\mathrm{T}}\left(Z_{\mathrm{P}}-2\right)}{R} .
$$

The operators $K_{j}$ and $h_{j}$ possess their eigen-problems:

$$
\begin{aligned}
& K_{i} \phi_{\boldsymbol{k}_{i}}^{+}\left(\boldsymbol{r}_{i}\right)=\frac{k_{i}^{2}}{2 \mu_{i}} \phi_{\boldsymbol{k}_{i}}^{+}\left(\boldsymbol{r}_{i}\right), h_{i} \varphi_{i}^{\mathrm{T}}\left(\boldsymbol{x}_{1}, \boldsymbol{x}_{2}\right)=\epsilon_{i}^{\mathrm{T}} \varphi_{i}^{\mathrm{T}}\left(\boldsymbol{x}_{1}, \boldsymbol{x}_{2}\right), \\
& K_{f} \phi_{-\boldsymbol{k}_{f}}^{-}\left(\boldsymbol{r}_{f}\right)=\frac{k_{f}^{2}}{2 \mu_{f}} \phi_{-\boldsymbol{k}_{f}}^{-}\left(\boldsymbol{r}_{f}\right), h_{f} \varphi_{f}^{\mathrm{P}}\left(\boldsymbol{s}_{1}, \boldsymbol{s}_{2}\right)=\epsilon_{f}^{\mathrm{P}} \varphi_{f}^{\mathrm{P}}\left(\boldsymbol{s}_{1}, \boldsymbol{s}_{2}\right),
\end{aligned}
$$

with the corresponding eigen-values and eigen-functions $\left\{k_{i}^{2} /\left(2 \mu_{i}\right), \phi_{\boldsymbol{k}_{i}}^{+}\left(\boldsymbol{r}_{i}\right)\right\}$, $\left\{k_{f}^{2} /\left(2 \mu_{f}\right), \phi_{-\boldsymbol{k}_{f}}^{-}\left(\boldsymbol{r}_{f}\right)\right\},\left\{\epsilon_{i}^{\mathrm{T}}, \varphi_{i}^{\mathrm{T}}\left(\boldsymbol{x}_{1}, \boldsymbol{x}_{2}\right)\right\}$ and $\left\{\epsilon_{f}^{\mathrm{P}}, \varphi_{f}^{\mathrm{P}}\left(\boldsymbol{s}_{1}, \boldsymbol{s}_{2}\right)\right\}$. Hereafter, the superscripts + and - denote the outgoing and incoming wave behaviors.

The unperturbed channel state (say $\Phi$ ) describes the two non-interacting aggregates (a nucleus and a heliumlike bound system). Therefore, the constituent component of $\Phi$ must contain the discrete heliumlike state vector $\varphi$ and the wavefunction $\phi$ of the relative motion of the free nucleus with respect to the c.m. of the remaining twoparticle bound system. The explicit way by which $\varphi$ and $\phi$ are combined to form $\Phi$ is prescribed by the probabilistic interpretation of a typical quantum-mechanical wavefunction. Therefore, since $\varphi$ and $\phi$ describe two independent sub-systems, $\Phi$ is 
given by the product of $\varphi$ and $\phi$, so that $\Phi=\varphi \phi$. This reasoning is supported by the quantitative analysis using the eigen-problems for the channel Hamiltonians $H_{i}$ and $H_{f}:$

$$
\begin{gathered}
H_{i} \Phi_{i}^{+}\left(\boldsymbol{x}_{1}, \boldsymbol{x}_{2}, \boldsymbol{r}_{i}\right)=E_{i} \Phi_{i}^{+}\left(\boldsymbol{x}_{1}, \boldsymbol{x}_{2}, \boldsymbol{r}_{i}\right), \\
H_{f} \Phi_{f}^{-}\left(\boldsymbol{s}_{1}, \boldsymbol{s}_{2}, \boldsymbol{r}_{f}\right)=E_{f} \Phi_{f}^{-}\left(\boldsymbol{s}_{1}, \boldsymbol{s}_{2}, \boldsymbol{r}_{f}\right),
\end{gathered}
$$

where $E_{i}$ and $E_{f}$ are the initial and final total energy of the whole system:

$$
E_{i}=\frac{k_{i}^{2}}{\mu_{i}}+\epsilon_{i}^{\mathrm{T}}, \quad E_{f}=\frac{k_{f}^{2}}{\mu_{f}}+\epsilon_{f}^{\mathrm{P}}, \quad E_{f}=E_{i} .
$$

The complete Schrödinger equations for the initial and final total scattering states $\Psi_{i}^{+}$ and $\Psi_{f}^{-}$are defined in terms of the same full Hamiltonian $H$ as:

$$
H \Psi_{i}^{+}=E_{i} \Psi_{i}^{+}, H \Psi_{f}^{-}=E_{f} \Psi_{f}^{-}
$$

Returning to the earlier defined final velocity vector $\boldsymbol{v}_{f}$, we can now determine the exact magnitude $v_{f}$ by using the energy conservation law $E_{f}=E_{i}$ from (2.14), which then gives:

$$
v_{f}=v_{i} \sqrt{\frac{\mu_{i}}{\mu_{f}}\left(1+\frac{\epsilon_{i}^{\mathrm{T}}-\epsilon_{f}^{\mathrm{P}}}{E_{i}}\right)} .
$$

This expression can conveniently be simplified for heavy nuclei $\mathrm{P}$ and $\mathrm{T}$ for which $M_{\mathrm{P}, \mathrm{T}} \gg 1$. If further $E_{i} \gg\left|\epsilon_{i}^{\mathrm{T}}-\epsilon_{f}^{\mathrm{P}}\right|$, which is true especially at intermediate and high energies, it follows from (2.16):

$$
v_{f} \approx v_{i}, M_{\mathrm{P}, \mathrm{T}} \gg 1, E_{i} \gg\left|\epsilon_{i}^{\mathrm{T}}-\epsilon_{f}^{\mathrm{P}}\right| \text {. }
$$

The scattering angle $\vartheta$ is defined by $\vartheta \equiv \cos ^{-1}\left(\hat{\boldsymbol{v}}_{i} \cdot \hat{\boldsymbol{v}}_{f}\right)$ where $\hat{\boldsymbol{v}}_{j}=\boldsymbol{v}_{j} / v_{j}(j=i, f)$. Due to their large mass, heavy projectile nuclei deviate only slightly from their incident direction. In practice, the largest values of $\vartheta$ are only a fraction of a milliradian (mrad) and, therefore, $\hat{\boldsymbol{v}}_{f} \approx \hat{\boldsymbol{v}}_{i}$, so that:

$$
\boldsymbol{v}_{f} \approx \boldsymbol{v}_{i}, M_{\mathrm{P}, \mathrm{T}} \gg 1, E_{i} \gg\left|\epsilon_{i}^{\mathrm{T}}-\epsilon_{f}^{\mathrm{P}}\right|
$$

Using the additive forms of $H_{i}$ as well as $H_{f}$ and employing the eigen-problems from (2.11), the mentioned factorizations of the channel wavefunctions are explicitly given by:

$$
\Phi_{i}^{+}\left(\boldsymbol{x}_{1}, \boldsymbol{x}_{2}, \boldsymbol{r}_{i}\right)=\phi_{\boldsymbol{k}_{i}}^{+}\left(\boldsymbol{r}_{i}\right) \varphi_{i}^{\mathrm{T}}\left(\boldsymbol{x}_{1}, \boldsymbol{x}_{2}\right)
$$




$$
\Phi_{f}^{-}\left(\boldsymbol{s}_{1}, \boldsymbol{s}_{2}, \boldsymbol{r}_{f}\right)=\phi_{-\boldsymbol{k}_{f}}^{-}\left(\boldsymbol{r}_{f}\right) \varphi_{f}^{\mathrm{P}}\left(\boldsymbol{s}_{1}, \boldsymbol{s}_{2}\right)
$$

Specifically, in only one particular case with nuclear charges $Z_{\mathrm{P}}=2=Z_{\mathrm{T}}$, as in double electron capture from helium by an alpha particle $\left(\mathrm{He}^{2+}-\mathrm{He}\right)$, function $\phi$ reduces to the associated plane wave. In this symmetric collision, we have in the entrance and exit channels:

$$
\phi_{\boldsymbol{k}_{i}}^{+}\left(\boldsymbol{r}_{i}\right)=\mathrm{e}^{i \boldsymbol{k}_{i} \cdot \boldsymbol{r}_{i}}, \phi_{-\boldsymbol{k}_{f}}^{-}\left(\boldsymbol{r}_{f}\right)=\mathrm{e}^{-i \boldsymbol{k}_{f} \cdot \boldsymbol{r}_{f}}
$$

However, in an asymmetric collision $\left(Z_{\mathrm{P}} \neq Z_{\mathrm{T}}\right)$, it follows that $\phi$ must be a Coulomb wave function for the relative motion of two heavy aggregates. In both channels, the free nucleus is conceived as interacting with a "reduced particle" of the effective charge $Z_{\mathrm{J}}-2(\mathrm{~J}=\mathrm{P}$ or $\mathrm{J}=\mathrm{T})$ and of the reduced mass $\mu_{j}(j=i$ or $j=f)$ located in the c.m. of $\left(\mathrm{T} ; e_{1}, e_{2}\right)_{i}$ or $\left(\mathrm{P} ; e_{1}, e_{2}\right)_{f}$.

These interactions in the entrance and exit channels are pure Coulomb potentials $\tilde{V}_{i}=Z_{\mathrm{P}}\left(Z_{\mathrm{T}}-2\right) / r_{i}$ and $\tilde{V}_{f}=Z_{\mathrm{T}}\left(Z_{\mathrm{P}}-2\right) / r_{f}$. Since in the eikonal approximation (the heavy mass limit), we have $r_{f} \approx r_{i} \approx R$, it follows, by reference to (2.10), that $\tilde{V}_{j} \approx V_{j}^{\infty}(j=i, f)$. Thus, in the general case of arbitrary nuclear charges $Z_{\mathrm{P}}$ and $Z_{\mathrm{P}}$, the functions $\phi_{\boldsymbol{k}_{i}}^{+}\left(\boldsymbol{r}_{i}\right)$ and $\phi_{-\boldsymbol{k}_{f}}^{-}\left(\boldsymbol{r}_{f}\right)$ represent the Coulomb waves:

$$
\begin{gathered}
\phi_{\boldsymbol{k}_{i}}^{+}\left(\boldsymbol{r}_{i}\right)=N^{+}\left(v_{i}\right) \mathrm{e}^{i \boldsymbol{k}_{i} \cdot \boldsymbol{r}_{i}}{ }_{1} F_{1}\left(-i v_{i}, 1, i k_{i} r_{i}-i \boldsymbol{k}_{i} \cdot \boldsymbol{r}_{i}\right), \\
N^{+}\left(v_{i}\right)=\mathrm{e}^{-\pi v_{i} / 2} \Gamma\left(1+i v_{i}\right), v_{i}=\frac{Z_{\mathrm{P}}\left(Z_{\mathrm{T}}-2\right)}{v_{i}}, \\
\phi_{-\boldsymbol{k}_{f}}^{-}\left(\boldsymbol{r}_{f}\right)=N^{-}\left(v_{f}\right) \mathrm{e}^{-i \boldsymbol{k}_{f} \cdot \boldsymbol{r}_{f}}{ }_{1} F_{1}\left(i v_{f}, 1,-i k_{f} \boldsymbol{r}_{f}+i \boldsymbol{k}_{f} \cdot \boldsymbol{r}_{f}\right), \\
N^{-}\left(v_{f}\right)=\mathrm{e}^{-\pi v_{f} / 2} \Gamma\left(1-i v_{f}\right), v_{f}=\frac{Z_{\mathrm{T}}\left(Z_{\mathrm{P}}-2\right)}{v_{f}},
\end{gathered}
$$

where the symbols $\Gamma$ and ${ }_{1} F_{1}$ stand for the gamma and confluent (Kummer) hypergeometric function, respectively. The Sommerfeld parameter $v_{j}(j=i$ or $j=f)$ is equal to zero only for $Z_{\mathrm{J}}=2(\mathrm{~J}=\mathrm{P}$ or $\mathrm{J}=\mathrm{T})$ in which case the pertinent Coulomb wavefunction becomes the corresponding plane wave so that Eqs. (2.21) and (2.23) coincide which other.

As opposed to excitation from the category of direct processes, double charge exchange belongs to more involved rearranging collisions. The specificity of process (2.1) is in the occurrence that neither of the two electrons remain bound to its parent nucleus in the final state. Rather, a rearrangement of particles takes place with both electrons being transferred from the target to the projectile nucleus. Without any loss of generality, it can be assumed that the target is initially at rest. In such a case, the relative velocity $\boldsymbol{v}_{i}$ becomes the incident velocity, which is denoted by $\boldsymbol{v}$ so that $\boldsymbol{v}_{i}=\boldsymbol{v}$. With this at hand, we can rewrite (2.18) as $\boldsymbol{v}_{f} \approx \boldsymbol{v}_{i} \equiv \boldsymbol{v}$.

For fast projectiles, two-electron transfer is expected to represent quite an unlikely event. However, the chance for both electrons to simultaneously jump onto the fast moving projectile nucleus, which passes by the target, will be significantly enhanced, 
if the electrons in the exit channel could escape from the field of their parent nucleus by receiving the momenta $\kappa_{e_{1}} \approx \kappa_{e_{2}}$ of the nearly equal magnitude and direction as $\boldsymbol{v}$.

However, this would be highly unlikely if double-electron capture is to take place in a direct one-step encounter of $Z_{\mathrm{P}}$ with $e_{1,2}$. This is the case because no momentum distribution of bound electrons in a heliumlike atomic systems could possess very large momenta, $m_{e} \boldsymbol{\kappa}_{e_{1,2}} \approx m_{e} \boldsymbol{v}$. The problem can be mitigated if both electrons were first ionized and then captured from the twofold continuum state. The electrons in the Coulomb field of $Z_{\mathrm{P}}$ can move with velocity $\boldsymbol{v}$ of the scattered projectile. Evidently, this would fulfill the momentum matching condition $\left(m_{e} \boldsymbol{\kappa}_{e_{1,2}} \approx m_{e} \boldsymbol{v}\right)$ for simultaneous capture of both electrons by $Z_{\mathrm{P}}$.

Physically, the relation $\boldsymbol{\kappa}_{e_{1,2}} \approx \boldsymbol{v}$ is recognized as a typical resonance effect which, in turn, increases the probability for the event of double capture. Hence, the probability for two-electron transfer would be considerably enhanced if, in an intermediate stage of collision, both electrons are first ionized from the target with the emission momenta $\boldsymbol{\kappa}_{e_{1,2}}$ equal to the velocity vector $\boldsymbol{v}_{f} \approx \boldsymbol{v}$ of the scattered projectile nucleus. This resonance mechanism greatly facilitates double capture, since the relative velocity vector $\Delta \boldsymbol{v}$ introduced as the difference between the velocity $\boldsymbol{v}_{f}$ of the scattered $Z_{\mathrm{P}}$ and the velocities $\boldsymbol{v}_{e_{1,2}}$ of the ejected electrons is nearly zero, $\Delta \boldsymbol{v} \equiv \boldsymbol{v}_{f}-\boldsymbol{v}_{e_{k}} \approx$ $\boldsymbol{v}-\boldsymbol{v}_{e_{k}} \approx \mathbf{0}(k=1,2)$.

In an experiment aimed at measuring cross sections for double charge exchange, a beam or incident nuclei is considered and, consequently, a beam of electrons is created prior to capture. Viewing these particle beams from the kinetic theory of gases, the definite average temperatures could be attributed to scattered projectile and intermediately ionized electrons. Then the mentioned resonance condition with the velocity matching could be recast into the equivalent near equality of the average temperatures of the stream of scattered projectiles and electrons ionized from the target. On the other hand, the occurrence of the nearly equal temperatures signifies the well-known adiabaticity conditions as the state of maximal coherence between the two particle beams, and this leads to an increased chance for double capture.

By reference to the spatial relationships of particles at the resonance condition $\boldsymbol{v}_{e} \approx \boldsymbol{v}$, the scattered projectile nucleus and the two electrons are conceived as being located near each other, so that their attractive Coulomb interactions are sufficient to bring them closer together into a bound heliumlike state. Another reason for including these continuum intermediate states of both electrons is the fact that double ionization dominates (by orders of magnitude) over double electron capture at high incident energies.

This is obvious from total cross sections $Q$ considered as a function of the increased impact energy $E$, since already single ionization (SI) has an inverse quasi-linear fall-off, $Q_{\mathrm{SI}} \sim E^{-1} \ln (E)$, in sharp contrast to the corresponding single capture (SC), where $Q_{\mathrm{SC}} \sim E^{-6}$ (without resonance, one step: the Oppenheimer-BrinkmanKramers mechanism) or $Q_{\mathrm{SC}} \sim E^{-11 / 2}$ (with resonance, two steps: the classical billiard-type Thomas mechanism). Therefore, at sufficiently high energies, capture probability could rise by including twofold electronic ionization continua as the distorted wave quantum-mechanical counterpart of the classical Thomas double scat- 
tering. This has abundantly been shown to be necessary for single capture [3], and a similar conclusion should also apply to double capture.

Consequently, the total scattering states $\Psi_{i, f}^{ \pm}$for double capture should contain a mixture of double continuum intermediate states of both electrons. Such a rationale is further supported by the fact that each of the two electrons reside simultaneously in the two Coulomb fields stemming from the target and projectile nucleus. This occurs symmetrically in the entrance and exit channel. In the entrance channel of process (2.1), the target nucleus $\mathrm{T}$ binds the two electrons, that are at the same time in their continuum states in the field of the projectile nucleus $\mathrm{P}$.

Likewise, after double capture, in the exit channel, the two electrons are bound to the projectile $\mathrm{P}$, but they are simultaneously in their continuum states in the field of the target nucleus T. In the distorted wave formalism, this is described as follows. The total scattering state $\Psi$ for the motion of three bound and one free particles in a given channel is approximated by a distorted wave $\chi$ in a factorized form as the product $\chi=\Phi \zeta$ where $\Phi$ is the unperturbed channel state and $\zeta$ is a distortion.

Under such circumstances, with the two active electrons $\left(e_{1,2}\right)$ and the two active nuclei $\left(Z_{\mathrm{P}}, Z_{\mathrm{T}}\right)$, function $\Phi$ should contain four states: $\varphi$ (bound), $\varphi_{\kappa_{e_{1}}}\left(e_{1}\right.$-nucleus continum $) \varphi_{\kappa_{e_{2}}}\left(e_{2}-\right.$ nucleus continum) and $\varphi_{\kappa_{\mathrm{PT}}}$ (nucleus-nucleus continuum). Here, the continuum states $\varphi_{\kappa_{\mathrm{PT}}}$ of the internuclear Coulomb potential $V_{\mathrm{PT}}$ for the relative motion of the two nuclei is also included, since one nucleus is always free in the field of the other nucleus in both channels.

In the entrance channel, the bound state is on the target, as described by the wavefunction $\varphi_{i}^{\mathrm{T}}\left(\boldsymbol{x}_{1}, \boldsymbol{x}_{2}\right)$, whereas the three continuum wavefunctions $\varphi_{-\boldsymbol{v}}^{+}\left(\boldsymbol{s}_{1}, \boldsymbol{s}_{2}\right)=$ $\varphi_{-v}^{+}\left(s_{1}\right) \varphi_{-v}^{+}\left(s_{2}\right)$ and $\varphi_{-\mu_{i} v}^{+}(\boldsymbol{R})$ of the two electrons and nucleus $\mathrm{T}$ are all centered on the projectile nucleus $\mathrm{P}$, according to:

$$
\Psi_{i}^{+} \approx\left\{\chi_{i}^{+}\right\}_{\text {initial distorted wave total scattering state }}
$$

$\chi_{i}^{+}=\left\{\Phi_{i}\right\}_{\text {unperturbed entrance channel state }}$

$\times\left\{\zeta_{i}^{+}\right\}_{\text {three Coulomb waves centered on P for electronic and nuclear motions }}$

$$
\begin{aligned}
\zeta_{i}^{+}= & \left\{\varphi_{-\boldsymbol{v}}^{+}\left(\boldsymbol{s}_{1}\right)\right\}_{V_{\mathrm{P}_{1}}} \text { : one Coulomb electronic continuum wave centered on } \mathrm{P} \\
& \times\left\{\varphi_{-\boldsymbol{v}}^{+}\left(\boldsymbol{s}_{2}\right)\right\}_{V_{\mathrm{P}_{2}}: \text { one Coulomb electronic continuum wave centered on } \mathrm{P}} \\
& \times\left\{\varphi_{-\mu_{i} \boldsymbol{v}}^{+}(-\boldsymbol{R})\right\}_{V_{\mathrm{PT}} \text { : one Coulomb wave for the motion of T relative to } \mathrm{P}}
\end{aligned}
$$

Symmetrically, in the exit channel, the bound state is on the scattered projectile, as described by the wavefunction $\varphi_{f}^{\mathrm{P}}\left(\boldsymbol{s}_{1}, \boldsymbol{s}_{2}\right)$, whereas the three continuum states $\varphi_{\boldsymbol{v}}^{-}\left(\boldsymbol{x}_{1}, \boldsymbol{x}_{2}\right)=\varphi_{\boldsymbol{v}}^{-}\left(\boldsymbol{x}_{1}\right) \varphi_{\boldsymbol{v}}^{-}\left(\boldsymbol{x}_{2}\right)$ and $\varphi_{\mu_{f}}^{-}(\boldsymbol{R})$ of the two electrons and nucleus $\mathrm{P}$ are all centered on the target nucleus $\mathrm{T}$ : 


$$
\begin{aligned}
\Psi_{f}^{-} \approx & \left\{\chi_{f}^{-}\right\}_{\text {final distorted wave total scattering state }} \\
\chi_{f}^{-}= & \left\{\Phi_{f}\right\}_{\text {unperturbed exit channel state }} \\
& \times\left\{\zeta_{f}^{-}\right\}_{\text {three Coulomb waves centered on } \mathrm{T} \text { for electronic and nuclear motions }} \\
\zeta_{f}^{-}= & \left\{\varphi_{\boldsymbol{v}}^{-}\left(\boldsymbol{x}_{1}\right)\right\}_{V_{\mathrm{T} e_{1}}: \text { one Coulomb electronic continuum wave centered on } \mathrm{T}} \\
& \times\left\{\varphi_{\boldsymbol{v}}^{-}\left(\boldsymbol{x}_{2}\right)\right\}_{V_{\mathrm{T} e_{2}}: \text { one Coulomb electronic continuum wave centered on } \mathrm{T}} \\
& \times\left\{\varphi_{\mu_{f} \boldsymbol{v}}^{-}(\boldsymbol{R})\right\}_{V_{\mathrm{PT}}: \text { one Coulomb wave for the motion of P relative to } \mathrm{T}} .
\end{aligned}
$$

Notice that $\chi_{f}^{-}$can be deduced from $\chi_{i}^{+}$by the following simultaneous changes:

$$
\chi_{f}^{-}=\left\{\chi_{i}^{+*}\right\}_{i \rightarrow f, \mathrm{P} \Longleftrightarrow \mathrm{T}, x_{1,2} \rightarrow s_{1,2}},
$$

and undoing the complex conjugation of the final heliumlike wavefunction by resetting $\varphi_{f}^{*} \rightarrow \varphi_{f}$ in the rhs of Eq. (2.30).

\section{Illustrations}

With the outlined succinct recapitulations of the basic features of the problem under consideration, it is clear that one-electron transfer in a pure three-body formalism can be extended to two-electron transfer in a pure four-body formalism without undue difficulty. In the distorted wave formalism, the explicit derivations of the transition amplitudes for double charge exchange in ion-atom collisions at intermediate and high energies for seven methods have recently been reported in Ref. [8] and, therefore, need not be repeated here. These include five theories of interest to the present applications: the CB1-4B, BCIS-4B, BDW-4B, CDW-4B and CDW-EIS-4B methods.

The computations in this work are carried out for the following symmetric, resonant ground-to-ground-state double charge exchange process:

$$
{ }^{4} \mathrm{He}^{2+}+{ }^{4} \mathrm{He}\left(1 \mathrm{~s}^{2}\right) \longrightarrow{ }^{4} \mathrm{He}\left(1 \mathrm{~s}^{2}\right)+{ }^{4} \mathrm{He}^{2+} .
$$

The available measurements on total cross sections for double charge exchange in the $\alpha-\mathrm{He}\left(1 s^{2}\right)$ collisions have been performed for capture into all helium bound states (ground and excited):

$$
{ }^{4} \mathrm{He}^{2+}+{ }^{4} \mathrm{He}\left(1 \mathrm{~s}^{2}\right) \longrightarrow{ }^{4} \mathrm{He}(\Sigma)+{ }^{4} \mathrm{He}^{2+} .
$$

Nevertheless, the theoretical results for (3.1) can still be compared with experimental data on (3.2) on account of a small contribution from singly and doubly excited final states of helium, as has been shown in the CDW-4B method [55]. In the illustrations, all the cross sections from the CB1-4B, BCIS-4B, BDW-4B, CDW-4B and CDWEIS method refer to the one-parameter Hylleraas' [95] ground-state wavefunction of 
helium with the configuration $(1 s)^{2}$ in both the entrance and exit channels. It has been shown in the CDW-4B method [39] that the total cross sections with this one-parameter wavefunction are similar to those with the 2-4 parameter $\left(1 s 1 s^{\prime}\right)$ wave functions that have the $\left(1 s 1 s^{\prime}\right)$ configurations. Of course, such a conclusion cannot automatically apply to the CB1-4B, BCIS-4B, BDW-4B and CDW-EIS-4B method without some explicit computations (needless to say, these are desirable to be performed in the near future).

The transition amplitudes in these methods are semi-analytical. Namely, the initial, defining nine-dimensional integrals are first reduced by analytical means to lower-dimensional integrals that are computed numerically. Finally, for the total cross sections $(Q)$, the absolute squared values of these transition amplitudes is also integrated numerically over the magnitude $\eta$ of the transverse momentum transfer vector $\eta$ (the integration of $\varphi_{\eta}$ is analytical with the result $2 \pi$ ). Overall, for computations of $Q$ in the general case of process (3.1) with the arbitrary nuclear charges $Z_{\mathrm{P}}$ and $Z_{\mathrm{T}}$, the number of the remaining numerical integrations is 3 (CB1-4B), 4 (CDW-4B, CDW-EIS-4B) and 5 (BCIS-4B, BDW-4B). The present computations employ the CB1-4B, BCIS-4B, BDW-4B and CDW-4B methods. The results from the CDW-EIS4B method are taken from Ref. [55].

All the numerical integrations in the CB1-4B, BCIS-4B, BDW-4B and CDW-4B methods are carried out by using the successive Gauss-Legendre quadrature rule with the varying order $N$ which is the number of pivots. These quadrature points or pivots are the zeros of the Legendre polynomial of degree $N$. The order $N$ has been varied in step of 16 beginning from $N=16$ all the way up to $N=192$. The ensuing results of the total cross sections are tabulated for $N=48,64, \ldots, 192$ and also shown graphically. The goal is to establish and visualize the convergence pattern of the displayed total cross sections $Q\left(\mathrm{~cm}^{2}\right)$ as a function the impact energy $E(\mathrm{keV})$ for the systematically and gradually increased values of $N$ in the increment of 16. This numerical experiment with respect to the dependence of $Q$ on $N$ is performed for all the considered impact energies $E \in[100,10000] \mathrm{keV}$.

The same order $N$ is used per each integration axis, i.e. for all the innermost integrals in the transition amplitudes as well as in the outermost integral over $\eta$ for the integration of the squared absolute values of the transition amplitudes. Integration over $\eta$ is appropriately scaled following Ref. [96] to acknowledge the fact that for heavy projectiles the most important contribution to total cross sections comes from the forward cone of scattering.

The numerical integrations in the CDW-4B method are straightforward as the transition amplitude (after the Fourier transform) is reduced to three-dimensional integrals in the momentum space (with the momentom variable $\tau$ ). The integration of the magnitude $\tau$ of vector $\boldsymbol{\tau}$ is scaled to the interval [0,1] after an appropriate change of the integration variable. The numerical integration in the transition amplitudes from the BCIS-4B and BDW-4B methods are done using the integral representations of the two Kummer confluent hypergeometric functions ${ }_{1} F_{1}$ defined in the intervals $t_{j} \in[0,1](j=1,2)$.

These Kummer functions contain the integrable singularities (branch points) at $t_{1,2}=0$ and $t_{1,2}=1$ that are smoothed out after an appropriate Cauchy regularization $[40,49,50]$. The Cauchy regularizations give the accurate results as verified 
by an alternative reduction of the 4-dimensional transition amplitudes in the BCIS$4 \mathrm{~B}$ and BDW-4B methods to the 3-dimensional integrals over the Gauss ordinary (non-confluent) hypergeometric function ${ }_{2} F_{1}$. This Gauss hypergeometric function ${ }_{2} F_{1}$ comes by analytical calculation over the two Kummer confluent hyppergeometric functions ${ }_{1} F_{1}$ instead of using their integral representations for the mentioned numerical quadratures.

At the impact energies $E \in[100,7000] \mathrm{keV}$, for the ten Gauss-Legendre orders $N=48, \ldots, 192$ the detailed total cross sections $Q_{\mathrm{BCIS}-4 \mathrm{~B}}$ and $Q_{\mathrm{BDW}-4 \mathrm{~B}}$ are given in Table 1, whereas Table 2 is for $Q_{\mathrm{CB} 1-4 \mathrm{~B}}$ and $Q_{\mathrm{CDW}-4 \mathrm{~B}}$ in the same range of $N$. It is seen from Table 1 that the values of $Q_{\mathrm{BCIS}-4 \mathrm{~B}}$ and $Q_{\mathrm{BDW}-4 \mathrm{~B}}$ stabilize for $N \geq 48$ at $E \geq 150 \mathrm{keV}$. Table 2 shows that $Q_{\mathrm{CB} 1-4 \mathrm{~B}}$ and $Q_{\mathrm{CDW}-4 \mathrm{~B}}$ are stable for $N \geq 48$ at $E \geq 100 \mathrm{keV}$. Table 3 summarizes $Q_{\mathrm{BCIS}-4 \mathrm{~B}}, Q_{\mathrm{BDW}-4 \mathrm{~B}}, Q_{\mathrm{CB} 1-4 \mathrm{~B}}$ and $Q_{\mathrm{CDW}-4 \mathrm{~B}}$ for $N=192$ at an extended energy interval $E \in[100,10000] \mathrm{keV}$.

The total cross sections from Tables 1, 2 and 3 are also visualized in Figs. 1, 2 and 3 and this is followed by Fig. 4 . To this end, Fig. 1 deals with $Q_{\mathrm{BCIS}-4 \mathrm{~B}}$ and $Q_{\mathrm{BDW}-4 \mathrm{~B}}$ for $N=48$, 64, 80, Fig. 2 for $N=96,112,128$ and Fig. 3 for $N=144,160,176$. In these figures, the results for $Q(N \leq 176)$ are compared with the fully stabilized cross sections $Q(N=192)$ that act as the reference data. The left and right columns of Figs. 1, 2 and 3 are for $Q_{\mathrm{BCIS}-4 \mathrm{~B}}$ and $Q_{\mathrm{BDW}-4 \mathrm{~B}}$, respectively. On each panel of both columns of Figs. 1, 2 and 3, open circles represent $Q_{\text {BCIS-4B }}$ and $Q_{\text {BDW-4B }}$ for $N \in[48,176]$. These are systematically compared with the full curves for the presently used highest quadrature order $N=192$, which itself secures full convergence at all impact energies $E \geq 150 \mathrm{keV}$. It can be observed from Figs. 1, 2 and 3 that at $E \geq 150$ $\mathrm{keV}$, the total cross sections $Q_{\mathrm{BCIS}-4 \mathrm{~B}}$ for $48 \leq N \leq 176$ on the left columns are indistinguishable from $Q_{\mathrm{BCIS}-4 \mathrm{~B}}$ for $N=192$. Moreover, in Figs. 1, 2 and 3, the same goes for $Q_{\mathrm{BDW}-4 \mathrm{~B}}$ on the right columns.

Overall, Figs. 1, 2 and 3 emphasize good convergence features of $Q_{\mathrm{BCIS}-4 \mathrm{~B}}$ and $Q_{\mathrm{BDW}-4 \mathrm{~B}}$ that exhibit robust stability against the Gauss-Legendre orders $N=$ $48, \ldots, 192$ at $E \geq 150 \mathrm{keV}$, as already noticed in Table 1 . This testifies to the reliability of the twofold Cauchy regularization of the mentioned double branch point singularities in each of the two integral representations of the Kummer confluent confluent hypergeometric functions.

The convergence characteristics of $Q_{\mathrm{CDW}-4 \mathrm{~B}}$ are not illustrated graphically since all the corresponding results for different $N$ would collapse onto the same data points on the scale of the figures, as implied by Table 2. The same remarkable convergence properties also hold true when plotting $Q_{\mathrm{CB} 1-4 \mathrm{~B}}$ (not shown). In particular, note that in the CDW-4B method, the Gauss-Legendre quadratures are used in three numerical integrations $\left\{\eta, \tau, \theta_{\boldsymbol{\tau}}\right\}$. Here, $\boldsymbol{\tau}=\left\{\tau, \theta_{\boldsymbol{\tau}}, \phi_{\boldsymbol{\tau}}\right\}$ is the momentum vector from the momentum representation of the product of the given bound-state orbital and the continuum Coulomb wavefunction. The remaining fourth numerical integration over $\phi_{\tau}$ in the CDW-4B method is performed by the Gauss-Mehler quadrature rule of varying order $M$. In this latter quadrature, our test computations at all the considered impact energies determined that the results for $Q_{\mathrm{CDW}-4 \mathrm{~B}}$ with $M=20$ and $M=40$ are the same. Therefore, in Tables 2 and 3, the numerical integration over $\phi_{\tau}$ was carried out with the Gauss-Mehler order $M=20$ at all impact energies. 
Table 1 Total cross sections $Q\left(\mathrm{~cm}^{2}\right)$ as a function of impact energy $E(\mathrm{keV})$ for double charge exchange ${ }^{4} \mathrm{He}^{2+}+{ }^{4} \mathrm{He}\left({ }^{1} \mathrm{~S}\right) \rightarrow{ }^{4} \mathrm{He}\left({ }^{1} \mathrm{~S}\right)+{ }^{4} \mathrm{He}^{2+}$ using ten Gauss-Legendre quadrature sets with orders $N=$ $48,64, \ldots, 192$ per each of five numerical integrations in the BCIS-4B and BDW-4B methods. Notation $2.2822,-17$ means $2.2822 \times 10^{-17} \mathrm{~cm}^{2}$

\begin{tabular}{|c|c|c|c|c|c|c|}
\hline $\begin{array}{l}\text { Method } \\
E(\mathrm{keV}) \\
N / Q\left(\mathrm{~cm}^{2}\right)\end{array}$ & $\begin{array}{l}\text { BCIS } \\
100\end{array}$ & $\begin{array}{l}\text { BDW } \\
100\end{array}$ & $\begin{array}{l}\text { BCIS } \\
110\end{array}$ & $\begin{array}{l}\text { BDW } \\
110\end{array}$ & $\begin{array}{l}\text { BCIS } \\
117.5\end{array}$ & $\begin{array}{l}\text { BDW } \\
117.5\end{array}$ \\
\hline 48 & $2.2822,-17$ & $1.6945,-17$ & $2.1153,-17$ & $1.6064,-17$ & $2.0505,-17$ & $1.6318,-17$ \\
\hline 64 & $2.9494,-17$ & $1.9069,-17$ & $2.6062,-17$ & $1.8714,-17$ & $2.4823,-17$ & $1.8476,-17$ \\
\hline 80 & $3.0206,-17$ & $1.9675,-17$ & $2.8403,-17$ & $1.9604,-17$ & $2.7177,-17$ & $1.9433,-17$ \\
\hline 96 & $2.7364,-17$ & $1.9141,-17$ & $2.8199,-17$ & $1.9733,-17$ & $2.7793,-17$ & $1.9755,-17$ \\
\hline 112 & $2.4943,-17$ & $1.8394,-17$ & $2.7108,-17$ & $1.9499,-17$ & $2.7469,-17$ & $1.9750,-17$ \\
\hline 128 & $2.3653,-17$ & $1.7880,-17$ & $2.6098,-17$ & $1.9194,-17$ & $2.6887,-17$ & $1.9612,-17$ \\
\hline 144 & $2.3157,-17$ & $1.7628,-17$ & $2.5419,-17$ & $1.8948,-17$ & $2.6361,-17$ & $1.9452,-17$ \\
\hline 160 & $2.3090,-17$ & $1.7550,-17$ & $2.5038,-17$ & $1.8786,-17$ & $2.5978,-17$ & $1.9316,-17$ \\
\hline 176 & $2.3223,-17$ & $1.7567,-17$ & $2.4865,-17$ & $1.8697,-17$ & $2.5733,-17$ & $1.9218,-17$ \\
\hline 192 & $2.3431,-17$ & $1.7626,-17$ & $2.4821,-17$ & $1.8659,-17$ & $2.5594,-17$ & $1.9154,-17$ \\
\hline $\begin{array}{l}\text { Method } \\
E(\mathrm{keV}) \\
N / Q\left(\mathrm{~cm}^{2}\right)\end{array}$ & $\begin{array}{l}\text { BCIS } \\
125\end{array}$ & $\begin{array}{l}\text { BDW } \\
125\end{array}$ & $\begin{array}{l}\text { BCIS } \\
137.5\end{array}$ & $\begin{array}{l}\text { BDW } \\
137.5\end{array}$ & $\begin{array}{l}\text { BCIS } \\
150\end{array}$ & $\begin{array}{l}\text { BDW } \\
150\end{array}$ \\
\hline 48 & $2.0797,-17$ & $1.7051,-17$ & $2.2435,-17$ & $1.8236,-17$ & $2.3865,-17$ & $1.8659,-17$ \\
\hline 64 & $2.4068,-17$ & $1.8318,-17$ & $2.3424,-17$ & $1.8193,-17$ & $2.3118,-17$ & $1.8003,-17$ \\
\hline 80 & $2.6196,-17$ & $1.9200,-17$ & $2.4933,-17$ & $1.8734,-17$ & $2.3910,-17$ & $1.8183,-17$ \\
\hline 96 & $2.7112,-17$ & $1.9595,-17$ & $2.5851,-17$ & $1.9094,-17$ & $2.4595,-17$ & $1.8416,-17$ \\
\hline 112 & $2.7241,-17$ & $1.9714,-17$ & $2.6270,-17$ & $1.9280,-17$ & $2.5020,-17$ & $1.8578,-17$ \\
\hline 128 & $2.7016,-17$ & $1.9693,-17$ & $2.6382,-17$ & $1.9356,-17$ & $2.5239,-17$ & $1.8673,-17$ \\
\hline 144 & $2.6700,-17$ & $1.9616,-17$ & $2.6336,-17$ & $1.9369,-17$ & $2.5325,-17$ & $1.8722,-17$ \\
\hline 160 & $2.6409,-17$ & $1.9528,-17$ & $2.6227,-17$ & $1.9351,-17$ & $2.5334,-17$ & $1.8741,-17$ \\
\hline 176 & $2.6183,-17$ & $1.9451,-17$ & $2.6104,-17$ & $1.9320,-17$ & $2.5304,-17$ & $1.8743,-17$ \\
\hline 192 & $2.6023,-17$ & $1.9390,-17$ & $2.5992,-17$ & $1.9287,-17$ & $2.5258,-17$ & $1.8736,-17$ \\
\hline $\begin{array}{l}\text { Method } \\
E(\mathrm{keV}) \\
N / Q\left(\mathrm{~cm}^{2}\right)\end{array}$ & $\begin{array}{l}\text { BCIS } \\
162.5\end{array}$ & $\begin{array}{l}\text { BDW } \\
162.5\end{array}$ & $\begin{array}{l}\text { BCIS } \\
175\end{array}$ & $\begin{array}{l}\text { BDW } \\
175\end{array}$ & $\begin{array}{l}\text { BCIS } \\
187.5\end{array}$ & $\begin{array}{l}\text { BDW } \\
187.5\end{array}$ \\
\hline 48 & $2.4276,-17$ & $1.8325,-17$ & $2.3734,-17$ & $1.7507,-17$ & $2.2568,-17$ & $1.6441,-17$ \\
\hline 64 & $2.2737,-17$ & $1.7566,-17$ & $2.2076,-17$ & $1.6865,-17$ & $2.1118,-17$ & $1.5967,-17$ \\
\hline 80 & $2.2942,-17$ & $1.7512,-17$ & $2.1912,-17$ & $1.6705,-17$ & $2.0777,-17$ & $1.5784,-17$ \\
\hline 96 & $2.3352,-17$ & $1.7615,-17$ & $2.2091,-17$ & $1.6717,-17$ & $2.0796,-17$ & $1.5745,-17$ \\
\hline 112 & $2.3678,-17$ & $1.7721,-17$ & $2.2299,-17$ & $1.6769,-17$ & $2.0902,-17$ & $1.5757,-17$ \\
\hline 128 & $2.3891,-17$ & $1.7800,-17$ & $2.2463,-17$ & $1.6820,-17$ & $2.1010,-17$ & $1.5784,-17$ \\
\hline 144 & $2.4015,-17$ & $1.7852,-17$ & $2.2577,-17$ & $1.6861,-17$ & $2.1098,-17$ & $1.5810,-17$ \\
\hline 160 & $2.4076,-17$ & $1.7883,-17$ & $2.2650,-17$ & $1.6890,-17$ & $2.1162,-17$ & $1.5832,-17$ \\
\hline 176 & $2.4099,-17$ & $1.7900,-17$ & $2.2693,-17$ & $1.6910,-17$ & $2.1206,-17$ & $1.5848,-17$ \\
\hline 192 & $2.4098,-17$ & $1.7907,-17$ & $2.2715,-17$ & $1.6922,-17$ & $2.1236,-17$ & $1.5861,-17$ \\
\hline
\end{tabular}


Table 1 continued

\begin{tabular}{|c|c|c|c|c|c|c|}
\hline $\begin{array}{l}\text { Method } \\
E(\mathrm{keV}) \\
N / Q\left(\mathrm{~cm}^{2}\right)\end{array}$ & $\begin{array}{l}\text { BCIS } \\
200\end{array}$ & $\begin{array}{l}\text { BDW } \\
200\end{array}$ & $\begin{array}{l}\text { BCIS } \\
225\end{array}$ & $\begin{array}{l}\text { BDW } \\
225\end{array}$ & $\begin{array}{l}\text { BCIS } \\
250\end{array}$ & $\begin{array}{l}\text { BDW } \\
250\end{array}$ \\
\hline 48 & $2.1076,-17$ & $1.5275,-17$ & $1.7846,-17$ & $1.2951,-17$ & $1.4836,-17$ & $1.0846,-17$ \\
\hline 64 & $1.9934,-17$ & $1.4953,-17$ & $1.7252,-17$ & $1.2833,-17$ & $1.4578,-17$ & $1.0826,-17$ \\
\hline 80 & $1.9545,-17$ & $1.4788,-17$ & $1.6937,-17$ & $1.2732,-17$ & $1.4381,-17$ & $1.0778,-17$ \\
\hline 96 & $1.9471,-17$ & $1.4728,-17$ & $1.6808,-17$ & $1.2677,-17$ & $1.4272,-17$ & $1.0742,-17$ \\
\hline 112 & $1.9504,-17$ & $1.4717,-17$ & $1.6768,-17$ & $1.2651,-17$ & $1.4220,-17$ & $1.0720,-17$ \\
\hline 128 & $1.9563,-17$ & $1.4725,-17$ & $1.6767,-17$ & $1.2641,-17$ & $1.4197,-17$ & $1.0708,-17$ \\
\hline 144 & $1.9621,-17$ & $1.4738,-17$ & $1.6782,-17$ & $1.2639,-17$ & $1.4191,-17$ & $1.0702,-17$ \\
\hline 160 & $1.9670,-17$ & $1.4752,-17$ & $1.6800,-17$ & $1.2641,-17$ & $1.4192,-17$ & $1.0699,-17$ \\
\hline 176 & $1.9707,-17$ & $1.4764,-17$ & $1.6819,-17$ & $1.2644,-17$ & $1.4197,-17$ & $1.0698,-17$ \\
\hline 192 & $1.9735,-17$ & $1.4774,-17$ & $1.6835,-17$ & $1.2648,-17$ & $1.4204,-17$ & $1.0698,-17$ \\
\hline $\begin{array}{l}\text { Method } \\
E(\mathrm{keV}) \\
N / Q\left(\mathrm{~cm}^{2}\right)\end{array}$ & $\begin{array}{l}\text { BCIS } \\
300\end{array}$ & $\begin{array}{l}\text { BDW } \\
300\end{array}$ & $\begin{array}{l}\text { BCIS } \\
350\end{array}$ & $\begin{array}{l}\text { BDW } \\
350\end{array}$ & $\begin{array}{l}\text { BCIS } \\
400\end{array}$ & $\begin{array}{l}\text { BDW } \\
400\end{array}$ \\
\hline 48 & $1.0098,-17$ & $7.5068,-18$ & $6.8761,-18$ & $5.1852,-18$ & $4.7205,-18$ & $3.6001,-18$ \\
\hline 64 & $1.0099,-17$ & $7.5400,-18$ & $6.9239,-18$ & $5.2137,-18$ & $4.7628,-18$ & $3.6172,-18$ \\
\hline 80 & $1.0048,-17$ & $7.5393,-18$ & $6.9224,-18$ & $5.2219,-18$ & $4.7728,-18$ & $3.6242,-18$ \\
\hline 96 & $1.0004,-17$ & $7.5315,-18$ & $6.9108,-18$ & $5.2231,-18$ & $4.7728,-18$ & $3.6269,-18$ \\
\hline 112 & $9.9732,-18$ & $7.5237,-18$ & $6.8993,-18$ & $5.2220,-18$ & $4.7700,-18$ & $3.6278,-18$ \\
\hline 128 & $9.9534,-18$ & $7.5175,-18$ & $6.8900,-18$ & $5.2204,-18$ & $4.7668,-18$ & $3.6279,-18$ \\
\hline 144 & $9.9412,-18$ & $7.5130,-18$ & $6.8831,-18$ & $5.2188,-18$ & $4.7639,-18$ & $3.6276,-18$ \\
\hline 160 & $9.9339,-18$ & $7.5099,-18$ & $6.8781,-18$ & $5.2174,-18$ & $4.7615,-18$ & $3.6273,-18$ \\
\hline 176 & $9.9299,-18$ & $7.5077,-18$ & $6.8745,-18$ & $5.2163,-18$ & $4.7595,-18$ & $3.6269,-18$ \\
\hline 192 & $9.9278,-18$ & $7.5062,-18$ & $6.8720,-18$ & $5.2154,-18$ & $4.7580,-18$ & $3.6265,-18$ \\
\hline $\begin{array}{l}\text { Method } \\
E(\mathrm{keV}) \\
N / Q\left(\mathrm{~cm}^{2}\right) \\
\end{array}$ & $\begin{array}{l}\text { BCIS } \\
450\end{array}$ & $\begin{array}{l}\text { BDW } \\
450\end{array}$ & $\begin{array}{l}\text { BCIS } \\
500\end{array}$ & $\begin{array}{l}\text { BDW } \\
500\end{array}$ & $\begin{array}{l}\text { BCIS } \\
550\end{array}$ & $\begin{array}{l}\text { BDW } \\
550\end{array}$ \\
\hline 48 & $3.2737,-18$ & $2.5194,-18$ & $2.2946,-18$ & $1.7792,-18$ & $1.6253,-18$ & $1.2685,-18$ \\
\hline 64 & $3.3028,-18$ & $2.5283,-18$ & $2.3129,-18$ & $1.7834,-18$ & $1.6364,-18$ & $1.2704,-18$ \\
\hline 80 & $3.3129,-18$ & $2.5328,-18$ & $2.3206,-18$ & $1.7860,-18$ & $1.6416,-18$ & $1.2717,-18$ \\
\hline 96 & $3.3159,-18$ & $2.5350,-18$ & $2.3237,-18$ & $1.7875,-18$ & $1.6441,-18$ & $1.2726,-18$ \\
\hline 112 & $3.3163,-18$ & $2.5361,-18$ & $2.3248,-18$ & $1.7883,-18$ & $1.6452,-18$ & $1.2731,-18$ \\
\hline 128 & $3.3156,-18$ & $2.5366,-18$ & $2.3251,-18$ & $1.7887,-18$ & $1.6457,-18$ & $1.2734,-18$ \\
\hline 144 & $3.3147,-18$ & $2.5367,-18$ & $2.3250,-18$ & $1.7890,-18$ & $1.6459,-18$ & $1.2736,-18$ \\
\hline 160 & $3.3138,-18$ & $2.5368,-18$ & $2.3248,-18$ & $1.7891,-18$ & $1.6460,-18$ & $1.2738,-18$ \\
\hline 176 & $3.3129,-18$ & $2.5367,-18$ & $2.3245,-18$ & $1.7892,-18$ & $1.6459,-18$ & $1.2739,-18$ \\
\hline 192 & $3.3122,-18$ & $2.5367,-18$ & $2.3242,-18$ & $1.7892,-18$ & $1.6458,-18$ & $1.2739,-18$ \\
\hline
\end{tabular}


Table 1 continued

\begin{tabular}{|c|c|c|c|c|c|c|}
\hline $\begin{array}{l}\text { Method } \\
E(\mathrm{keV}) \\
N / Q\left(\mathrm{~cm}^{2}\right)\end{array}$ & $\begin{array}{l}\text { BCIS } \\
600\end{array}$ & $\begin{array}{l}\text { BDW } \\
600\end{array}$ & $\begin{array}{l}\text { BCIS } \\
625\end{array}$ & $\begin{array}{l}\text { BDW } \\
625\end{array}$ & $\begin{array}{l}\text { BCIS } \\
650\end{array}$ & $\begin{array}{l}\text { BDW } \\
650\end{array}$ \\
\hline 48 & $1.1630,-18$ & $9.1556,-19$ & $9.8752,-19$ & $7.7758,-19$ & $8.4052,-19$ & $6.6368,-19$ \\
\hline 64 & $1.1697,-18$ & $9.1385,-19$ & $9.9263,-19$ & $7.7792,-19$ & $8.4444,-19$ & $6.6381,-19$ \\
\hline 80 & $1.1731,-18$ & $9.1453,-19$ & $9.9536,-19$ & $7.7839,-19$ & $8.4662,-19$ & $6.6413,-19$ \\
\hline 96 & $1.1749,-18$ & $9.1501,-19$ & $9.9682,-19$ & $7.7875,-19$ & $8.4782,-19$ & $6.6439,-19$ \\
\hline 112 & $1.1758,-18$ & $9.1534,-19$ & $9.9762,-19$ & $7.7900,-19$ & $8.4850,-19$ & $6.6458,-19$ \\
\hline 128 & $1.1763,-18$ & $9.1556,-19$ & $9.9806,-19$ & $7.7917,-19$ & $8.4889,-19$ & $6.6471,-19$ \\
\hline 144 & $1.1765,-18$ & $9.1570,-19$ & $9.9830,-19$ & $7.7929,-19$ & $8.4911,-19$ & $6.6481,-19$ \\
\hline 160 & $1.1766,-18$ & $9.1580,-19$ & $9.9843,-19$ & $7.7938,-19$ & $8.4924,-19$ & $6.6488,-19$ \\
\hline 176 & $1.1767,-18$ & $9.1587,-19$ & $9.9849,-19$ & $7.7943,-19$ & $8.4931,-19$ & $6.6493,-19$ \\
\hline 192 & $1.1767,-18$ & $9.1591,-19$ & $9.9852,-19$ & $7.7948,-19$ & $8.4935,-19$ & $6.6496,-19$ \\
\hline $\begin{array}{l}\text { Method } \\
E(\mathrm{keV}) \\
N / Q\left(\mathrm{~cm}^{2}\right)\end{array}$ & $\begin{array}{l}\text { BCIS } \\
700\end{array}$ & $\begin{array}{l}\text { BDW } \\
700\end{array}$ & $\begin{array}{l}\text { BCIS } \\
750\end{array}$ & $\begin{array}{l}\text { BDW } \\
750\end{array}$ & $\begin{array}{l}\text { BCIS } \\
800\end{array}$ & $\begin{array}{l}\text { BDW } \\
800\end{array}$ \\
\hline 48 & $6.1321,-19$ & $4.8686,-19$ & $4.5144,-19$ & $3.6037,-19$ & $3.3522,-19$ & $2.6905,-19$ \\
\hline 64 & $6.1550,-19$ & $4.8677,-19$ & $4.5276,-19$ & $3.6020,-19$ & $3.3597,-19$ & $2.6888,-19$ \\
\hline 80 & $6.1687,-19$ & $4.8689,-19$ & $4.5362,-19$ & $3.6023,-19$ & $3.3651,-19$ & $2.6887,-19$ \\
\hline 96 & $6.1768,-19$ & $4.8702,-19$ & $4.5415,-19$ & $3.6029,-19$ & $3.3686,-19$ & $2.6889,-19$ \\
\hline 112 & $6.1816,-19$ & $4.8713,-19$ & $4.5449,-19$ & $3.6035,-19$ & $3.3709,-19$ & $2.6892,-19$ \\
\hline 128 & $6.1845,-19$ & $4.8721,-19$ & $4.5469,-19$ & $3.6040,-19$ & $3.3723,-19$ & $2.6894,-19$ \\
\hline 144 & $6.1863,-19$ & $4.8727,-19$ & $4.5483,-19$ & $3.6043,-19$ & $3.3733,-19$ & $2.6897,-19$ \\
\hline 160 & $6.1874,-19$ & $4.8731,-19$ & $4.5492,-19$ & $3.6046,-19$ & $3.3740,-19$ & $2.6898,-19$ \\
\hline 176 & $6.1881,-19$ & $4.8735,-19$ & $4.5498,-19$ & $3.6048,-19$ & $3.3745,-19$ & $2.6900,-19$ \\
\hline 192 & $6.1885,-19$ & $4.8737,-19$ & $4.5502,-19$ & $3.6050,-19$ & $3.3748,-19$ & $2.6901,-19$ \\
\hline $\begin{array}{l}\text { Method } \\
E(\mathrm{keV}) \\
N / Q\left(\mathrm{~cm}^{2}\right)\end{array}$ & $\begin{array}{l}\text { BCIS } \\
850\end{array}$ & $\begin{array}{l}\text { BDW } \\
850\end{array}$ & $\begin{array}{l}\text { BCIS } \\
900\end{array}$ & $\begin{array}{l}\text { BDW } \\
900\end{array}$ & $\begin{array}{l}\text { BCIS } \\
950\end{array}$ & $\begin{array}{l}\text { BDW } \\
950\end{array}$ \\
\hline 48 & $2.5097,-19$ & $2.0254,-19$ & $1.8938,-19$ & $1.5368,-19$ & $1.4397,-19$ & $1.1749,-19$ \\
\hline 64 & $2.5139,-19$ & $2.0239,-19$ & $1.8960,-19$ & $1.5355,-19$ & $1.4408,-19$ & $1.1739,-19$ \\
\hline 80 & $2.5173,-19$ & $2.0236,-19$ & $1.8981,-19$ & $1.5352,-19$ & $1.4420,-19$ & $1.1735,-19$ \\
\hline 96 & $2.5196,-19$ & $2.0236,-19$ & $1.8996,-19$ & $1.5351,-19$ & $1.4430,-19$ & $1.1735,-19$ \\
\hline 112 & $2.5211,-19$ & $2.0237,-19$ & $1.9006,-19$ & $1.5352,-19$ & $1.4437,-19$ & $1.1734,-19$ \\
\hline 128 & $2.5221,-19$ & $2.0239,-19$ & $1.9013,-19$ & $1.5352,-19$ & $1.4442,-19$ & $1.1735,-19$ \\
\hline 144 & $2.5228,-19$ & $2.0240,-19$ & $1.9018,-19$ & $1.5353,-19$ & $1.4446,-19$ & $1.1735,-19$ \\
\hline 160 & $2.5233,-19$ & $2.0241,-19$ & $1.9022,-19$ & $1.5354,-19$ & $1.4448,-19$ & $1.1735,-19$ \\
\hline 176 & $2.5237,-19$ & $2.0242,-19$ & $1.9024,-19$ & $1.5354,-19$ & $1.4450,-19$ & $1.1736,-19$ \\
\hline 192 & $2.5239,-19$ & $2.0243,-19$ & $1.9026,-19$ & $1.5355,-19$ & $1.4452,-19$ & $1.1736,-19$ \\
\hline
\end{tabular}


Table 1 continued

\begin{tabular}{|c|c|c|c|c|c|c|}
\hline $\begin{array}{l}\text { Method } \\
E(\mathrm{keV}) \\
N / Q\left(\mathrm{~cm}^{2}\right)\end{array}$ & $\begin{array}{l}\text { BCIS } \\
1000\end{array}$ & $\begin{array}{l}\text { BDW } \\
1000\end{array}$ & $\begin{array}{l}\text { BCIS } \\
1250\end{array}$ & $\begin{array}{l}\text { BDW } \\
1250\end{array}$ & $\begin{array}{l}\text { BCIS } \\
1500\end{array}$ & $\begin{array}{l}\text { BDW } \\
1500\end{array}$ \\
\hline 48 & $1.1022,-19$ & $9.0469,-20$ & $3.1898,-20$ & $2.6968,-20$ & $1.0561,-20$ & $9.2104,-21$ \\
\hline 64 & $1.1027,-19$ & $9.0385,-20$ & $3.1877,-20$ & $2.6946,-20$ & $1.0549,-20$ & $9.2044,-21$ \\
\hline 80 & $1.1035,-19$ & $9.0358,-20$ & $3.1879,-20$ & $2.6937,-20$ & $1.0546,-20$ & $9.2016,-21$ \\
\hline 96 & $1.1041,-19$ & $9.0348,-20$ & $3.1885,-20$ & $2.6932,-20$ & $1.0546,-20$ & $9.2002,-21$ \\
\hline 112 & $1.1046,-19$ & $9.0346,-20$ & $3.1891,-20$ & $2.6930,-20$ & $1.0546,-20$ & $9.1993,-21$ \\
\hline 128 & $1.1049,-19$ & $9.0347,-20$ & $3.1896,-20$ & $2.6929,-20$ & $1.0547,-20$ & $9.1989,-21$ \\
\hline 144 & $1.1052,-19$ & $9.0348,-20$ & $3.1901,-20$ & $2.6928,-20$ & $1.0548,-20$ & $9.1986,-21$ \\
\hline 160 & $1.1054,-19$ & $9.0350,-20$ & $3.1904,-20$ & $2.6928,-20$ & $1.0548,-20$ & $9.1984,-21$ \\
\hline 176 & $1.1055,-19$ & $9.0351,-20$ & $3.1907,-20$ & $2.6928,-20$ & $1.0549,-20$ & $9.1982,-21$ \\
\hline 192 & $1.1056,-19$ & $9.0353,-20$ & $3.1909,-20$ & $2.6928,-20$ & $1.0549,-20$ & $9.1982,-21$ \\
\hline $\begin{array}{l}\text { Method } \\
E(\mathrm{keV}) \\
N / Q\left(\mathrm{~cm}^{2}\right)\end{array}$ & $\begin{array}{l}\text { BCIS } \\
2500\end{array}$ & $\begin{array}{l}\text { BDW } \\
2500\end{array}$ & $\begin{array}{l}\text { BCIS } \\
2750\end{array}$ & $\begin{array}{l}\text { BDW } \\
2750\end{array}$ & $\begin{array}{l}\text { BCIS } \\
3000\end{array}$ & $\begin{array}{l}\text { BDW } \\
3000\end{array}$ \\
\hline 48 & $3.8974,-21$ & $3.5092,-21$ & $1.5728,-21$ & $1.4624,-21$ & $6.8400,-22$ & $6.5664,-22$ \\
\hline 64 & $3.8923,-21$ & $3.5075,-21$ & $1.5707,-21$ & $1.4618,-21$ & $6.8311,-22$ & $6.5646,-22$ \\
\hline 80 & $3.8907,-21$ & $3.5066,-21$ & $1.5699,-21$ & $1.4616,-21$ & $6.8276,-22$ & $6.5636,-22$ \\
\hline 96 & $3.8902,-21$ & $3.5062,-21$ & $1.5696,-21$ & $1.4614,-21$ & $6.8261,-22$ & $6.5630,-22$ \\
\hline 112 & $3.8901,-21$ & $3.5059,-21$ & $1.5695,-21$ & $1.4613,-21$ & $6.8254,-22$ & $6.5626,-22$ \\
\hline 128 & $3.8901,-21$ & $3.5057,-21$ & $1.5694,-21$ & $1.4612,-21$ & $6.8250,-22$ & $6.5624,-22$ \\
\hline 144 & $3.8902,-21$ & $3.5056,-21$ & $1.5694,-21$ & $1.4612,-21$ & $6.8248,-22$ & $6.5622,-22$ \\
\hline 160 & $3.8903,-21$ & $3.5055,-21$ & $1.5694,-21$ & $1.4612,-21$ & $6.8248,-22$ & $6.5621,-22$ \\
\hline 176 & $3.8904,-21$ & $3.5055,-21$ & $1.5694,-21$ & $1.4611,-21$ & $6.8248,-22$ & $6.5620,-22$ \\
\hline 192 & $3.8905,-21$ & $3.5054,-21$ & $1.5695,-21$ & $1.4611,-21$ & $6.8248,-22$ & $6.5619,-22$ \\
\hline $\begin{array}{l}\text { Method } \\
E(\mathrm{keV}) \\
N / Q\left(\mathrm{~cm}^{2}\right)\end{array}$ & $\begin{array}{l}\text { BCIS } \\
2500\end{array}$ & $\begin{array}{l}\text { BDW } \\
2500\end{array}$ & $\begin{array}{l}\text { BCIS } \\
2750\end{array}$ & $\begin{array}{l}\text { BDW } \\
2750\end{array}$ & $\begin{array}{l}\text { BCIS } \\
3000\end{array}$ & $\begin{array}{l}\text { BDW } \\
3000\end{array}$ \\
\hline 48 & $3.1701,-22$ & $3.1405,-22$ & $1.5521,-22$ & $1.5854,-22$ & $7.9723,-23$ & $8.3861,-23$ \\
\hline 64 & $3.1662,-22$ & $3.1398,-22$ & $1.5504,-22$ & $1.5851,-22$ & $7.9639,-23$ & $8.3851,-23$ \\
\hline 80 & $3.1646,-22$ & $3.1395,-22$ & $1.5496,-22$ & $1.5850,-22$ & $7.9604,-23$ & $8.3845,-23$ \\
\hline 96 & $3.1639,-22$ & $3.1392,-22$ & $1.5493,-22$ & $1.5849,-22$ & $7.9586,-23$ & $8.3841,-23$ \\
\hline 112 & $3.1635,-22$ & $3.1391,-22$ & $1.5491,-22$ & $1.5848,-22$ & $7.9577,-23$ & $8.3838,-23$ \\
\hline 128 & $3.1633,-22$ & $3.1390,-22$ & $1.5490,-22$ & $1.5848,-22$ & $7.9571,-23$ & $8.3836,-23$ \\
\hline 144 & $3.1632,-22$ & $3.1389,-22$ & $1.5489,-22$ & $1.5847,-22$ & $7.9568,-23$ & $8.3835,-23$ \\
\hline 160 & $3.1632,-22$ & $3.1389,-22$ & $1.5489,-22$ & $1.5847,-22$ & $7.9566,-23$ & $8.3834,-23$ \\
\hline 176 & $3.1631,-22$ & $3.1388,-22$ & $1.5489,-22$ & $1.5847,-22$ & $7.9565,-23$ & $8.3833,-23$ \\
\hline 192 & $3.1631,-22$ & $3.1388,-22$ & $1.5488,-22$ & $1.5847,-22$ & $7.9564,-23$ & $8.3832,-23$ \\
\hline
\end{tabular}


Table 1 continued

\begin{tabular}{|c|c|c|c|c|c|c|}
\hline $\begin{array}{l}\text { Method } \\
E(\mathrm{keV}) \\
N / Q\left(\mathrm{~cm}^{2}\right)\end{array}$ & $\begin{array}{l}\text { BCIS } \\
3250\end{array}$ & $\begin{array}{l}\text { BDW } \\
3250\end{array}$ & $\begin{array}{l}\text { BCIS } \\
3500\end{array}$ & $\begin{array}{l}\text { BDW } \\
3500\end{array}$ & $\begin{array}{l}\text { BCIS } \\
3750\end{array}$ & $\begin{array}{l}\text { BDW } \\
3750\end{array}$ \\
\hline 48 & $4.2717,-23$ & $4.6210,-23$ & $2.3765,-23$ & $2.6396,-23$ & $1.3677,-23$ & $1.5566,-23$ \\
\hline 64 & $4.2675,-23$ & $4.6205,-23$ & $2.3746,-23$ & $2.6394,-23$ & $1.3669,-23$ & $1.5566,-23$ \\
\hline 80 & $4.2658,-23$ & $4.6202,-23$ & $2.3737,-23$ & $2.6392,-23$ & $1.3664,-23$ & $1.5565,-23$ \\
\hline 96 & $4.2649,-23$ & $4.6201,-23$ & $2.3733,-23$ & $2.6391,-23$ & $1.3662,-23$ & $1.5565,-23$ \\
\hline 112 & $4.2644,-23$ & $4.6199,-23$ & $2.3730,-23$ & $2.6391,-23$ & $1.3661,-23$ & $1.5564,-23$ \\
\hline 128 & $4.2642,-23$ & $4.6198,-23$ & $2.3729,-23$ & $2.6390,-23$ & $1.3666,-23$ & $1.5564,-23$ \\
\hline 144 & $4.2640,-23$ & $4.6198,-23$ & $2.3728,-23$ & $2.6390,-23$ & $1.3659,-23$ & $1.5564,-23$ \\
\hline 160 & $4.2638,-23$ & $4.6197,-23$ & $2.3727,-23$ & $2.6390,-23$ & $1.3659,-23$ & $1.5564,-23$ \\
\hline 176 & $4.2638,-23$ & $4.6197,-23$ & $2.3727,-23$ & $2.6390,-23$ & $1.3659,-23$ & $1.5564,-23$ \\
\hline 192 & $4.2639,-23$ & $4.6197,-23$ & $2.3727,-23$ & $2.6389,-23$ & $1.3659,-23$ & $1.5564,-23$ \\
\hline $\begin{array}{l}\text { Method } \\
E(\mathrm{keV}) \\
N / Q\left(\mathrm{~cm}^{2}\right)\end{array}$ & $\begin{array}{l}\text { BCIS } \\
4000\end{array}$ & $\begin{array}{l}\text { BDW } \\
4000\end{array}$ & $\begin{array}{l}\text { BCIS } \\
4250\end{array}$ & $\begin{array}{l}\text { BDW } \\
4250\end{array}$ & $\begin{array}{l}\text { BCIS } \\
4500\end{array}$ & $\begin{array}{l}\text { BDW } \\
4500\end{array}$ \\
\hline 48 & $8.1200,-24$ & $9.4456,-24$ & $4.9591,-24$ & $5.8815,-24$ & $3.1056,-24$ & $3.7481,-24$ \\
\hline 64 & $8.1134,-24$ & $9.4456,-24$ & $4.9533,-24$ & $5.8805,-24$ & $3.1034,-24$ & $3.7468,-24$ \\
\hline 80 & $8.1111,-24$ & $9.4452,-24$ & $4.9521,-24$ & $5.8804,-24$ & $3.1027,-24$ & $3.7467,-24$ \\
\hline 96 & $8.1100,-24$ & $9.4450,-24$ & $4.9515,-24$ & $5.8802,-24$ & $3.1023,-24$ & $3.7466,-24$ \\
\hline 112 & $8.1093,-24$ & $9.4448,-24$ & $4.9512,-24$ & $5.8801,-24$ & $3.1022,-24$ & $3.7466,-24$ \\
\hline 128 & $8.1089,-24$ & $9.4447,-24$ & $4.9510,-24$ & $5.8801,-24$ & $3.1021,-24$ & $3.7465,-24$ \\
\hline 144 & $8.1087,-24$ & $9.4446,-24$ & $4.9508,-24$ & $5.8800,-24$ & $3.1020,-24$ & $3.7465,-24$ \\
\hline 160 & $8.1085,-24$ & $9.4446,-24$ & $4.9508,-24$ & $5.8800,-24$ & $3.1020,-24$ & $3.7465,-24$ \\
\hline 176 & $8.1084,-24$ & $9.4445,-24$ & $4.9507,-24$ & $5.8800,-24$ & $3.1019,-24$ & $3.7465,-24$ \\
\hline 192 & $8.1083,-24$ & $9.4445,-24$ & $4.9507,-24$ & $5.8799,-24$ & $3.1019,-24$ & $3.7465,-24$ \\
\hline $\begin{array}{l}\text { Method } \\
E(\mathrm{keV}) \\
N / Q\left(\mathrm{~cm}^{2}\right)\end{array}$ & $\begin{array}{l}\text { BCIS } \\
4750\end{array}$ & $\begin{array}{l}\text { BDW } \\
4750\end{array}$ & $\begin{array}{l}\text { BCIS } \\
5000\end{array}$ & $\begin{array}{l}\text { BDW } \\
5000\end{array}$ & $\begin{array}{l}\text { BCIS } \\
5500\end{array}$ & $\begin{array}{l}\text { BDW } \\
5500\end{array}$ \\
\hline 48 & $1.9899,-24$ & $2.4385,-24$ & $1.3049,-24$ & $1.6162,-24$ & $6.0211,-25$ & $7.4758,-25$ \\
\hline 64 & $1.9915,-24$ & $2.4380,-24$ & $1.3066,-24$ & $1.6171,-24$ & $5.9825,-25$ & $7.4862,-25$ \\
\hline 80 & $1.9911,-24$ & $2.4379,-24$ & $1.3065,-24$ & $1.6170,-24$ & $5.9815,-25$ & $7.4870,-25$ \\
\hline 96 & $1.9909,-24$ & $2.4379,-24$ & $1.3064,-24$ & $1.6170,-24$ & $5.9814,-25$ & $7.4869,-25$ \\
\hline 112 & $1.9908,-24$ & $2.4378,-24$ & $1.3064,-24$ & $1.6170,-24$ & $5.9813,-25$ & $7.4869,-25$ \\
\hline 128 & $1.9908,-24$ & $2.4378,-24$ & $1.3064,-24$ & $1.6169,-24$ & $5.9813,-25$ & $7.4868,-25$ \\
\hline 144 & $1.9907,-24$ & $2.4378,-24$ & $1.3064,-24$ & $1.6169,-24$ & $5.9813,-25$ & $7.4868,-25$ \\
\hline 160 & $1.9907,-24$ & $2.4378,-24$ & $1.3064,-24$ & $1.6169,-24$ & $5.9813,-25$ & $7.4868,-25$ \\
\hline 176 & $1.9907,-24$ & $2.4378,-24$ & $1.3064,-24$ & $1.6169,-24$ & $5.9813,-25$ & $7.4868,-25$ \\
\hline 192 & $1.9907,-24$ & $2.4378,-24$ & $1.3064,-24$ & $1.6169,-24$ & $5.9813,-25$ & $7.4867,-25$ \\
\hline
\end{tabular}


Table 1 continued

\begin{tabular}{lllllll}
\hline $\begin{array}{l}\text { Method } \\
E(\mathrm{keV})\end{array}$ & BCIS & BDW & BCIS & BDW & BCIS & BDW \\
$N / Q\left(\mathrm{~cm}^{2}\right)$ & 6000 & 6000 & 6500 & 6500 & 7000 & 7000 \\
\hline 48 & & & & & & \\
64 & $2.9523,-25$ & $3.6886,-25$ & $1.5059,-25$ & $1.9145,-25$ & $8.9475,-26$ & $1.0260,-25$ \\
80 & $2.9497,-25$ & $3.6794,-25$ & $1.5463,-25$ & $1.9038,-25$ & $8.6907,-26$ & $1.0278,-25$ \\
96 & $2.9485,-25$ & $3.6792,-25$ & $1.5514,-25$ & $1.9028,-25$ & $8.6666,-26$ & $1.0283,-25$ \\
112 & $2.9483,-25$ & $3.6792,-25$ & $1.5519,-25$ & $1.9027,-25$ & $8.6624,-26$ & $1.0284,-25$ \\
128 & $2.9483,-25$ & $3.6792,-25$ & $1.5519,-25$ & $1.9027,-25$ & $8.6621,-26$ & $1.0284,-25$ \\
144 & $2.9483,-25$ & $3.6792,-25$ & $1.5520,-25$ & $1.9027,-25$ & $8.6623,-26$ & $1.0284,-25$ \\
160 & $2.9484,-25$ & $3.6792,-25$ & $1.5520,-25$ & $1.9027,-25$ & $8.6625,-26$ & $1.0284,-25$ \\
176 & $2.9484,-25$ & $3.6792,-25$ & $1.5520,-25$ & $1.9027,-25$ & $8.6626,-26$ & $1.0284,-25$ \\
192 & $2.9484,-25$ & $3.6792,-25$ & $1.5520,-25$ & $1.9027,-25$ & $8.6627,-26$ & $1.0284,-25$ \\
& $2.9484,-25$ & $3.6792,-25$ & $1.5520,-25$ & $1.9027,-25$ & $8.6628,-26$ & $1.0284,-25$ \\
\hline
\end{tabular}

Next, we pass onto a possible relationship between the BCIS-4B and BDW-4B methods. Prior to this, it would be useful to recall a link between the BCIS-3B and BDW-3B methods for a pure three-body charge exchange:

$$
Z_{\mathrm{P}}+\left(Z_{\mathrm{T}} ; e\right)_{i} \longrightarrow\left(Z_{\mathrm{P}} ; e\right)_{f}+Z_{\mathrm{T}}
$$

For this process, the cross sections in the BCIS-3B and BDW-3B methods are identical because the semi-analytical calculations of the transition amplitudes in these two theories yield the same expressions. This occurs despite the fact that the perturbation operators in the transition amplitudes are different in these two methods. The difference in the interaction operators is due to the applications of the defining perturbations (the full Hamiltonian minus the channel Hamiltonian) to two different total scattering wavefunctions (those for the initial and final states). Such a difference is only formal as one is free to apply the said defining perturbation potentials in the transition amplitudes to either the initial or the final total scattering states. The key for explaining the coincidence of the transition amplitudes in the BCIS-3B and BDW-3B methods is the availability of the exact initial and final bound states of the hydrogenlike atomic systems $\left(Z_{\mathrm{T}} ; e\right)_{i}$ and $\left(Z_{\mathrm{P}} ; e\right)_{f}$.

By contrast, no exact bound-state wavefunctions of heliumlike atomic systems exists $^{1}$. It is for this reason that the transition amplitudes in BCIS-4B and BDW-4B methods for double charge exchange (2.1) do not give the same analytical expression for any approximate bound-state heliumlike wavefunctions. This would occur for any form of the available approximate two-electron bound-state wavefunctions no matter how precise the corresponding variational binding energies can be.

\footnotetext{
${ }^{1}$ Here, the clause 'exact' refers to the finite number of operations. This term is conceptually different from e.g. the numerically most accurate heliumlike binding energy, irrespective of the number of the obtained decimal places. The reason is that all such results stem from some variational computations with iterations that do not terminate after a finite number of cycles.
} 
Table 2 Total cross sections $Q\left(\mathrm{~cm}^{2}\right)$ as a function of impact energy $E(\mathrm{keV})$ for double charge exchange ${ }^{4} \mathrm{He}^{2+}+{ }^{4} \mathrm{He}\left({ }^{1} \mathrm{~S}\right) \rightarrow{ }^{4} \mathrm{He}\left({ }^{1} \mathrm{~S}\right)+{ }^{4} \mathrm{He}^{2+}$ using ten Gauss-Legendre quadrature sets with orders $\mathrm{N}=48,64$, ...,192 per each of five numerical integrations in the CB1-4B and CDW-4B methods. Notation 3.5898,-16 means $3.5898 \times 10^{-16} \mathrm{~cm}^{2}$

\begin{tabular}{|c|c|c|c|c|c|c|}
\hline $\begin{array}{l}\text { Method } \\
E(\mathrm{keV}) \\
N / Q\left(\mathrm{~cm}^{2}\right)\end{array}$ & $\begin{array}{l}\text { CB1 } \\
100\end{array}$ & $\begin{array}{l}\text { CDW } \\
100\end{array}$ & $\begin{array}{l}\text { CB1 } \\
110\end{array}$ & $\begin{array}{l}\text { CDW } \\
110\end{array}$ & $\begin{array}{l}\text { CB1 } \\
117.5\end{array}$ & $\begin{array}{l}\text { CDW } \\
117.5\end{array}$ \\
\hline 48 & $3.5898,-16$ & $5.3139,-16$ & $3.0486,-16$ & $3.8247,-16$ & $2.7132,-16$ & $3.0380,-16$ \\
\hline 64 & $3.5898,-16$ & $5.3139,-16$ & $3.0486,-16$ & $3.8247,-16$ & $2.7132,-16$ & $3.0380,-16$ \\
\hline 80 & $3.5898,-16$ & $5.3139,-16$ & $3.0486,-16$ & $3.8247,-16$ & $2.7132,-16$ & $3.0380,-16$ \\
\hline 96 & $3.5898,-16$ & $5.3139,-16$ & $3.0486,-16$ & $3.8247,-16$ & $2.7132,-16$ & $3.0380,-16$ \\
\hline 112 & $3.5898,-16$ & $5.3139,-16$ & $3.0486,-16$ & $3.8247,-16$ & $2.7132,-16$ & $3.0380,-16$ \\
\hline 128 & $3.5898,-16$ & $5.3139,-16$ & $3.0486,-16$ & $3.8247,-16$ & $2.7132,-16$ & $3.0380,-16$ \\
\hline 144 & $3.5898,-16$ & $5.3139,-16$ & $3.0486,-16$ & $3.8247,-16$ & $2.7132,-16$ & $3.0380,-16$ \\
\hline 160 & $3.5898,-16$ & $5.3139,-16$ & $3.0486,-16$ & $3.8247,-16$ & $2.7132,-16$ & $3.0380,-16$ \\
\hline 176 & $3.5898,-16$ & $5.3139,-16$ & $3.0486,-16$ & $3.8247,-16$ & $2.7132,-16$ & $3.0380,-16$ \\
\hline 192 & $3.5898,-16$ & $5.3139,-16$ & $3.0486,-16$ & $3.8247,-16$ & $2.7132,-16$ & $3.0380,-16$ \\
\hline $\begin{array}{l}\text { Method } \\
E(\mathrm{keV}) \\
N / Q\left(\mathrm{~cm}^{2}\right)\end{array}$ & $\begin{array}{l}\text { CB1 } \\
125\end{array}$ & $\begin{array}{l}\text { CDW } \\
125\end{array}$ & $\begin{array}{l}\text { CB1 } \\
137.5\end{array}$ & $\begin{array}{l}\text { CDW } \\
137.5\end{array}$ & $\begin{array}{l}\text { CB1 } \\
150\end{array}$ & $\begin{array}{l}\text { CDW } \\
150\end{array}$ \\
\hline 48 & $2.4256,-16$ & $2.4425,-16$ & $2.0299,-16$ & $1.7375,-16$ & $1.7148,-16$ & $1.2668,-16$ \\
\hline 64 & $2.4256,-16$ & $2.4425,-16$ & $2.0299,-16$ & $1.7375,-16$ & $1.7148,-16$ & $1.2668,-16$ \\
\hline 80 & $2.4256,-16$ & $2.4425,-16$ & $2.0299,-16$ & $1.7375,-16$ & $1.7148,-16$ & $1.2668,-16$ \\
\hline 96 & $2.4256,-16$ & $2.4425,-16$ & $2.0299,-16$ & $1.7375,-16$ & $1.7148,-16$ & $1.2668,-16$ \\
\hline 112 & $2.4256,-16$ & $2.4425,-16$ & $2.0299,-16$ & $1.7375,-16$ & $1.7148,-16$ & $1.2668,-16$ \\
\hline 128 & $2.4256,-16$ & $2.4425,-16$ & $2.0299,-16$ & $1.7375,-16$ & $1.7148,-16$ & $1.2668,-16$ \\
\hline 144 & $2.4256,-16$ & $2.4425,-16$ & $2.0299,-16$ & $1.7375,-16$ & $1.7148,-16$ & $1.2668,-16$ \\
\hline 160 & $2.4256,-16$ & $2.4425,-16$ & $2.0299,-16$ & $1.7375,-16$ & $1.7148,-16$ & $1.2668,-16$ \\
\hline 176 & $2.4256,-16$ & $2.4425,-16$ & $2.0299,-16$ & $1.7375,-16$ & $1.7148,-16$ & $1.2668,-16$ \\
\hline 192 & $2.4256,-16$ & $2.4425,-16$ & $2.0299,-16$ & $1.7375,-16$ & $1.7148,-16$ & $1.2668,-16$ \\
\hline $\begin{array}{l}\text { Method } \\
E(\mathrm{keV}) \\
N / Q\left(\mathrm{~cm}^{2}\right)\end{array}$ & $\begin{array}{l}\text { CB1 } \\
162.5\end{array}$ & $\begin{array}{l}\text { CDW } \\
162.5\end{array}$ & $\begin{array}{l}\text { CB1 } \\
175\end{array}$ & $\begin{array}{l}\text { CDW } \\
175\end{array}$ & $\begin{array}{l}\text { CB1 } \\
187.5\end{array}$ & $\begin{array}{l}\text { CDW } \\
187.5\end{array}$ \\
\hline 48 & $1.4603,-16$ & $9.4296,-17$ & $1.2523,-16$ & $7.1438,-17$ & $1.0805,-16$ & $5.4952,-17$ \\
\hline 64 & $1.4603,-16$ & $9.4296,-17$ & $1.2523,-16$ & $7.1438,-17$ & $1.0805,-16$ & $5.4952,-17$ \\
\hline 80 & $1.4603,-16$ & $9.4296,-17$ & $1.2523,-16$ & $7.1438,-17$ & $1.0805,-16$ & $5.4952,-17$ \\
\hline 96 & $1.4603,-16$ & $9.4296,-17$ & $1.2523,-16$ & $7.1438,-17$ & $1.0805,-16$ & $5.4952,-17$ \\
\hline 112 & $1.4603,-16$ & $9.4296,-17$ & $1.2523,-16$ & $7.1438,-17$ & $1.0805,-16$ & $5.4952,-17$ \\
\hline 128 & $1.4603,-16$ & $9.4296,-17$ & $1.2523,-16$ & $7.1438,-17$ & $1.0805,-16$ & $5.4952,-17$ \\
\hline 144 & $1.4603,-16$ & $9.4296,-17$ & $1.2523,-16$ & $7.1438,-17$ & $1.0805,-16$ & $5.4952,-17$ \\
\hline 160 & $1.4603,-16$ & $9.4296,-17$ & $1.2523,-16$ & $7.1438,-17$ & $1.0805,-16$ & $5.4952,-17$ \\
\hline 176 & $1.4603,-16$ & $9.4296,-17$ & $1.2523,-16$ & $7.1438,-17$ & $1.0805,-16$ & $5.4952,-17$ \\
\hline 192 & $1.4603,-16$ & $9.4296,-17$ & $1.2523,-16$ & $7.1438,-17$ & $1.0805,-16$ & $5.4952,-17$ \\
\hline
\end{tabular}


Table 2 continued

\begin{tabular}{|c|c|c|c|c|c|c|}
\hline $\begin{array}{l}\text { Method } \\
E(\mathrm{keV}) \\
N / Q\left(\mathrm{~cm}^{2}\right)\end{array}$ & $\begin{array}{l}\text { CB1 } \\
200\end{array}$ & $\begin{array}{l}\text { CDW } \\
200\end{array}$ & $\begin{array}{l}\text { CB1 } \\
225\end{array}$ & $\begin{array}{l}\text { CDW } \\
225\end{array}$ & $\begin{array}{l}\text { CB1 } \\
250\end{array}$ & $\begin{array}{l}\text { CDW } \\
250\end{array}$ \\
\hline 48 & $9.3737,-17$ & $4.2838,-17$ & $7.1556,-17$ & $2.6932,-17$ & $5.5513,-17$ & $1.7582,-17$ \\
\hline 64 & $9.3737,-17$ & $4.2838,-17$ & $7.1556,-17$ & $2.6932,-17$ & $5.5513,-17$ & $1.7582,-17$ \\
\hline 80 & $9.3737,-17$ & $4.2838,-17$ & $7.1556,-17$ & $2.6932,-17$ & $5.5513,-17$ & $1.7582,-17$ \\
\hline 96 & $9.3737,-17$ & $4.2838,-17$ & $7.1556,-17$ & $2.6932,-17$ & $5.5513,-17$ & $1.7582,-17$ \\
\hline 112 & $9.3737,-17$ & $4.2838,-17$ & $7.1556,-17$ & $2.6932,-17$ & $5.5513,-17$ & $1.7582,-17$ \\
\hline 128 & $9.3737,-17$ & $4.2838,-17$ & $7.1556,-17$ & $2.6932,-17$ & $5.5513,-17$ & $1.7582,-17$ \\
\hline 144 & $9.3737,-17$ & $4.2838,-17$ & $7.1556,-17$ & $2.6932,-17$ & $5.5513,-17$ & $1.7582,-17$ \\
\hline 160 & $9.3737,-17$ & $4.2838,-17$ & $7.1556,-17$ & $2.6932,-17$ & $5.5513,-17$ & $1.7582,-17$ \\
\hline 176 & $9.3737,-17$ & $4.2838,-17$ & $7.1556,-17$ & $2.6932,-17$ & $5.5513,-17$ & $1.7582,-17$ \\
\hline 192 & $9.3737,-17$ & $4.2838,-17$ & $7.1556,-17$ & $2.6932,-17$ & $5.5513,-17$ & $1.7582,-17$ \\
\hline $\begin{array}{l}\text { Method } \\
E(\mathrm{keV}) \\
N / Q\left(\mathrm{~cm}^{2}\right)\end{array}$ & $\begin{array}{l}\text { CB1 } \\
300\end{array}$ & $\begin{array}{l}\text { CDW } \\
300\end{array}$ & $\begin{array}{l}\text { CB1 } \\
350\end{array}$ & $\begin{array}{l}\text { CDW } \\
350\end{array}$ & $\begin{array}{l}\text { CB1 } \\
400\end{array}$ & $\begin{array}{l}\text { CDW } \\
400\end{array}$ \\
\hline 48 & $3.4738,-17$ & $8.1755,-18$ & $2.2653,-17$ & $4.1526,-18$ & $1.5263,-17$ & $2.2542,-18$ \\
\hline 64 & $3.4738,-17$ & $8.1755,-18$ & $2.2653,-17$ & $4.1526,-18$ & $1.5263,-17$ & $2.2542,-18$ \\
\hline 80 & $3.4738,-17$ & $8.1755,-18$ & $2.2653,-17$ & $4.1526,-18$ & $1.5263,-17$ & $2.2542,-18$ \\
\hline 96 & $3.4738,-17$ & $8.1755,-18$ & $2.2653,-17$ & $4.1526,-18$ & $1.5263,-17$ & $2.2542,-18$ \\
\hline 112 & $3.4738,-17$ & $8.1755,-18$ & $2.2653,-17$ & $4.1526,-18$ & $1.5263,-17$ & $2.2542,-18$ \\
\hline 128 & $3.4738,-17$ & $8.1755,-18$ & $2.2653,-17$ & $4.1526,-18$ & $1.5263,-17$ & $2.2542,-18$ \\
\hline 144 & $3.4738,-17$ & $8.1755,-18$ & $2.2653,-17$ & $4.1526,-18$ & $1.5263,-17$ & $2.2542,-18$ \\
\hline 160 & $3.4738,-17$ & $8.1755,-18$ & $2.2653,-17$ & $4.1526,-18$ & $1.5263,-17$ & $2.2542,-18$ \\
\hline 176 & $3.4738,-17$ & $8.1755,-18$ & $2.2653,-17$ & $4.1526,-18$ & $1.5263,-17$ & $2.2542,-18$ \\
\hline 192 & $3.4738,-17$ & $8.1755,-18$ & $2.2653,-17$ & $4.1526,-18$ & $1.5263,-17$ & $2.2542,-18$ \\
\hline $\begin{array}{l}\text { Method } \\
E(\mathrm{keV}) \\
N / Q\left(\mathrm{~cm}^{2}\right)\end{array}$ & $\begin{array}{l}\text { CB1 } \\
450\end{array}$ & $\begin{array}{l}\text { CDW } \\
450\end{array}$ & $\begin{array}{l}\text { CB1 } \\
500\end{array}$ & $\begin{array}{l}\text { CDW } \\
500\end{array}$ & $\begin{array}{l}\text { CB1 } \\
550\end{array}$ & $\begin{array}{l}\text { CDW } \\
550\end{array}$ \\
\hline 48 & $1.0563,-17$ & $1.2894,-18$ & $7.4774,-18$ & $7.6957,-19$ & $5.3971,-18$ & $4.7593,-19$ \\
\hline 64 & $1.0563,-17$ & $1.2894,-18$ & $7.4774,-18$ & $7.6957,-19$ & $5.3971,-18$ & $4.7593,-19$ \\
\hline 80 & $1.0563,-17$ & $1.2894,-18$ & $7.4774,-18$ & $7.6957,-19$ & $5.3971,-18$ & $4.7593,-19$ \\
\hline 96 & $1.0563,-17$ & $1.2894,-18$ & $7.4774,-18$ & $7.6957,-19$ & $5.3971,-18$ & $4.7593,-19$ \\
\hline 112 & $1.0563,-17$ & $1.2894,-18$ & $7.4774,-18$ & $7.6957,-19$ & $5.3971,-18$ & $4.7593,-19$ \\
\hline 128 & $1.0563,-17$ & $1.2894,-18$ & $7.4774,-18$ & $7.6957,-19$ & $5.3971,-18$ & $4.7593,-19$ \\
\hline 144 & $1.0563,-17$ & $1.2894,-18$ & $7.4774,-18$ & $7.6957,-19$ & $5.3971,-18$ & $4.7593,-19$ \\
\hline 160 & $1.0563,-17$ & $1.2894,-18$ & $7.4774,-18$ & $7.6957,-19$ & $5.3971,-18$ & $4.7593,-19$ \\
\hline 176 & $1.0563,-17$ & $1.2894,-18$ & $7.4774,-18$ & $7.6957,-19$ & $5.3971,-18$ & $4.7593,-19$ \\
\hline 192 & $1.0563,-17$ & $1.2894,-18$ & $7.4774,-18$ & $7.6957,-19$ & $5.3971,-18$ & $4.7593,-19$ \\
\hline
\end{tabular}


Table 2 continued

\begin{tabular}{|c|c|c|c|c|c|c|}
\hline $\begin{array}{l}\text { Method } \\
E(\mathrm{keV}) \\
N / Q\left(\mathrm{~cm}^{2}\right)\end{array}$ & $\begin{array}{l}\text { CB1 } \\
600\end{array}$ & $\begin{array}{l}\text { CDW } \\
600\end{array}$ & $\begin{array}{l}\text { CB1 } \\
625\end{array}$ & $\begin{array}{l}\text { CDW } \\
625\end{array}$ & $\begin{array}{l}\text { CB1 } \\
650\end{array}$ & $\begin{array}{l}\text { CDW } \\
650\end{array}$ \\
\hline 48 & $3.9624,-18$ & $3.0336,-19$ & $3.4146,-18$ & $2.4464,-19$ & $2.9531,-18$ & $1.9849,-19$ \\
\hline 64 & $3.9624,-18$ & $3.0336,-19$ & $3.4146,-18$ & $2.4464,-19$ & $2.9531,-18$ & $1.9849,-19$ \\
\hline 80 & $3.9624,-18$ & $3.0336,-19$ & $3.4146,-18$ & $2.4464,-19$ & $2.9531,-18$ & $1.9849,-19$ \\
\hline 96 & $3.9624,-18$ & $3.0336,-19$ & $3.4146,-18$ & $2.4464,-19$ & $2.9531,-18$ & $1.9849,-19$ \\
\hline 112 & $3.9624,-18$ & $3.0336,-19$ & $3.4146,-18$ & $2.4464,-19$ & $2.9531,-18$ & $1.9849,-19$ \\
\hline 128 & $3.9624,-18$ & $3.0336,-19$ & $3.4146,-18$ & $2.4464,-19$ & $2.9531,-18$ & $1.9849,-19$ \\
\hline 144 & $3.9624,-18$ & $3.0336,-19$ & $3.4146,-18$ & $2.4464,-19$ & $2.9531,-18$ & $1.9849,-19$ \\
\hline 160 & $3.9624,-18$ & $3.0336,-19$ & $3.4146,-18$ & $2.4464,-19$ & $2.9531,-18$ & $1.9849,-19$ \\
\hline 176 & $3.9624,-18$ & $3.0336,-19$ & $3.4146,-18$ & $2.4464,-19$ & $2.9531,-18$ & $1.9849,-19$ \\
\hline 192 & $3.9624,-18$ & $3.0336,-19$ & $3.4146,-18$ & $2.4464,-19$ & $2.9531,-18$ & $1.9849,-19$ \\
\hline $\begin{array}{l}\text { Method } \\
E(\mathrm{keV}) \\
N / Q\left(\mathrm{~cm}^{2}\right)\end{array}$ & $\begin{array}{l}\text { CB1 } \\
700\end{array}$ & $\begin{array}{l}\text { CDW } \\
700\end{array}$ & $\begin{array}{l}\text { CB1 } \\
750\end{array}$ & $\begin{array}{l}\text { CDW } \\
750\end{array}$ & $\begin{array}{l}\text { CB1 } \\
800\end{array}$ & $\begin{array}{l}\text { CDW } \\
800\end{array}$ \\
\hline 48 & $2.2306,-18$ & $1.3288,-19$ & $1.7053,-18$ & $9.0780,-20$ & $1.3181,-18$ & $6.3154,-20$ \\
\hline 64 & $2.2306,-18$ & $1.3288,-19$ & $1.7053,-18$ & $9.0780,-20$ & $1.3181,-18$ & $6.3153,-20$ \\
\hline 80 & $2.2306,-18$ & $1.3288,-19$ & $1.7053,-18$ & $9.0780,-20$ & $1.3181,-18$ & $6.3153,-20$ \\
\hline 96 & $2.2306,-18$ & $1.3288,-19$ & $1.7053,-18$ & $9.0780,-20$ & $1.3181,-18$ & $6.3153,-20$ \\
\hline 112 & $2.2306,-18$ & $1.3288,-19$ & $1.7053,-18$ & $9.0780,-20$ & $1.3181,-18$ & $6.3153,-20$ \\
\hline 128 & $2.2306,-18$ & $1.3288,-19$ & $1.7053,-18$ & $9.0780,-20$ & $1.3181,-18$ & $6.3153,-20$ \\
\hline 144 & $2.2306,-18$ & $1.3288,-19$ & $1.7053,-18$ & $9.0780,-20$ & $1.3181,-18$ & $6.3153,-20$ \\
\hline 160 & $2.2306,-18$ & $1.3288,-19$ & $1.7053,-18$ & $9.0780,-20$ & $1.3181,-18$ & $6.3153,-20$ \\
\hline 176 & $2.2306,-18$ & $1.3288,-19$ & $1.7053,-18$ & $9.0780,-20$ & $1.3181,-18$ & $6.3153,-20$ \\
\hline 192 & $2.2306,-18$ & $1.3288,-19$ & $1.7053,-18$ & $9.0780,-20$ & $1.3181,-18$ & $6.3153,-20$ \\
\hline $\begin{array}{l}\text { Method } \\
E(\mathrm{keV}) \\
N / Q\left(\mathrm{~cm}^{2}\right)\end{array}$ & $\begin{array}{l}\text { CB } 1 \\
850\end{array}$ & $\begin{array}{l}\text { CDW } \\
850\end{array}$ & $\begin{array}{l}\text { CB1 } \\
900\end{array}$ & $\begin{array}{l}\text { CDW } \\
900\end{array}$ & $\begin{array}{l}\text { CB1 } \\
950\end{array}$ & $\begin{array}{l}\text { CDW } \\
950\end{array}$ \\
\hline 48 & $1.0290,-18$ & $4.4658,-20$ & $8.1066,-19$ & $3.2050,-20$ & $6.4406,-19$ & $2.3314,-20$ \\
\hline 64 & $1.0290,-18$ & $4.4658,-20$ & $8.1066,-19$ & $3.2050,-20$ & $6.4406,-19$ & $2.3314,-20$ \\
\hline 80 & $1.0290,-18$ & $4.4658,-20$ & $8.1066,-19$ & $3.2050,-20$ & $6.4406,-19$ & $2.3314,-20$ \\
\hline 96 & $1.0290,-18$ & $4.4658,-20$ & $8.1066,-19$ & $3.2050,-20$ & $6.4406,-19$ & $2.3314,-20$ \\
\hline 112 & $1.0290,-18$ & $4.4658,-20$ & $8.1066,-19$ & $3.2050,-20$ & $6.4406,-19$ & $2.3314,-20$ \\
\hline 128 & $1.0290,-18$ & $4.4658,-20$ & $8.1066,-19$ & $3.2050,-20$ & $6.4406,-19$ & $2.3314,-20$ \\
\hline 144 & $1.0290,-18$ & $4.4658,-20$ & $8.1066,-19$ & $3.2050,-20$ & $6.4406,-19$ & $2.3314,-20$ \\
\hline 160 & $1.0290,-18$ & $4.4658,-20$ & $8.1066,-19$ & $3.2050,-20$ & $6.4406,-19$ & $2.3314,-20$ \\
\hline 176 & $1.0290,-18$ & $4.4658,-20$ & $8.1066,-19$ & $3.2050,-20$ & $6.4406,-19$ & $2.3314,-20$ \\
\hline 192 & $1.0290,-18$ & $4.4658,-20$ & $8.1066,-19$ & $3.2050,-20$ & $6.4406,-19$ & $2.3314,-20$ \\
\hline
\end{tabular}


Table 2 continued

\begin{tabular}{|c|c|c|c|c|c|c|}
\hline $\begin{array}{l}\text { Method } \\
E(\mathrm{keV}) \\
N / Q\left(\mathrm{~cm}^{2}\right)\end{array}$ & $\begin{array}{l}\text { CB1 } \\
1000\end{array}$ & $\begin{array}{l}\text { CDW } \\
1000\end{array}$ & $\begin{array}{l}\text { CB1 } \\
1250\end{array}$ & $\begin{array}{l}\text { CDW } \\
1250\end{array}$ & $\begin{array}{l}\text { CB1 } \\
1500\end{array}$ & $\begin{array}{l}\text { CDW } \\
1500\end{array}$ \\
\hline 48 & $5.1568,-19$ & $1.7171,-20$ & $1.8732,-19$ & $4.3434,-21$ & $7.7542,-20$ & $1.3425,-21$ \\
\hline 64 & $5.1568,-19$ & $1.7170,-20$ & $1.8732,-19$ & $4.3433,-21$ & $7.7542,-20$ & $1.3425,-21$ \\
\hline 80 & $5.1568,-19$ & $1.7170,-20$ & $1.8732,-19$ & $4.3433,-21$ & $7.7542,-20$ & $1.3425,-21$ \\
\hline 96 & $5.1568,-19$ & $1.7170,-20$ & $1.8732,-19$ & $4.3433,-21$ & $7.7542,-20$ & $1.3425,-21$ \\
\hline 112 & $5.1568,-19$ & $1.7170,-20$ & $1.8732,-19$ & $4.3433,-21$ & $7.7542,-20$ & $1.3425,-21$ \\
\hline 128 & $5.1568,-19$ & $1.7170,-20$ & $1.8732,-19$ & $4.3433,-21$ & $7.7542,-20$ & $1.3425,-21$ \\
\hline 144 & $5.1568,-19$ & $1.7170,-20$ & $1.8732,-19$ & $4.3433,-21$ & $7.7542,-20$ & $1.3425,-21$ \\
\hline 160 & $5.1568,-19$ & $1.7170,-20$ & $1.8732,-19$ & $4.3433,-21$ & $7.7542,-20$ & $1.3425,-21$ \\
\hline 176 & $5.1568,-19$ & $1.7170,-20$ & $1.8732,-19$ & $4.3433,-21$ & $7.7542,-20$ & $1.3425,-21$ \\
\hline 192 & $5.1568,-19$ & $1.7170,-20$ & $1.8732,-19$ & $4.3433,-21$ & $7.7542,-20$ & $1.3425,-21$ \\
\hline $\begin{array}{l}\text { Method } \\
E(\mathrm{keV}) \\
N / Q\left(\mathrm{~cm}^{2}\right)\end{array}$ & $\begin{array}{l}\text { CB1 } \\
2500\end{array}$ & $\begin{array}{l}\text { CDW } \\
2500\end{array}$ & $\begin{array}{l}\text { CB1 } \\
2750\end{array}$ & $\begin{array}{l}\text { CDW } \\
2750\end{array}$ & $\begin{array}{l}\text { CB1 } \\
3000\end{array}$ & $\begin{array}{l}\text { CDW } \\
3000\end{array}$ \\
\hline 48 & $3.5428,-20$ & $4.8094,-22$ & $1.7495,-20$ & $1.9296,-22$ & $9.2007,-21$ & $8.4772,-23$ \\
\hline 64 & $3.5428,-20$ & $4.8097,-22$ & $1.7495,-20$ & $1.9301,-22$ & $9.2007,-21$ & $8.4761,-23$ \\
\hline 80 & $3.5428,-20$ & $4.8096,-22$ & $1.7495,-20$ & $1.9301,-22$ & $9.2007,-21$ & $8.4761,-23$ \\
\hline 96 & $3.5428,-20$ & $4.8096,-22$ & $1.7495,-20$ & $1.9301,-22$ & $9.2007,-21$ & $8.4761,-23$ \\
\hline 112 & $3.5428,-20$ & $4.8096,-22$ & $1.7495,-20$ & $1.9301,-22$ & $9.2007,-21$ & $8.4761,-23$ \\
\hline 128 & $3.5428,-20$ & $4.8096,-22$ & $1.7495,-20$ & $1.9301,-22$ & $9.2007,-21$ & $8.4761,-23$ \\
\hline 144 & $3.5428,-20$ & $4.8096,-22$ & $1.7495,-20$ & $1.9301,-22$ & $9.2007,-21$ & $8.4761,-23$ \\
\hline 160 & $3.5428,-20$ & $4.8096,-22$ & $1.7495,-20$ & $1.9301,-22$ & $9.2007,-21$ & $8.4761,-23$ \\
\hline 176 & $3.5428,-20$ & $4.8096,-22$ & $1.7495,-20$ & $1.9301,-22$ & $9.2007,-21$ & $8.4761,-23$ \\
\hline 192 & $3.5428,-20$ & $4.8096,-22$ & $1.7495,-20$ & $1.9301,-22$ & $9.2007,-21$ & $8.4761,-23$ \\
\hline $\begin{array}{l}\text { Method } \\
E(\mathrm{keV}) \\
N / Q\left(\mathrm{~cm}^{2}\right)\end{array}$ & $\begin{array}{l}\text { CB1 } \\
2500\end{array}$ & $\begin{array}{l}\text { CDW } \\
2500\end{array}$ & $\begin{array}{l}\text { CB1 } \\
2750\end{array}$ & $\begin{array}{l}\text { CDW } \\
2750\end{array}$ & $\begin{array}{l}\text { CB1 } \\
3000\end{array}$ & $\begin{array}{l}\text { CDW } \\
3000\end{array}$ \\
\hline 48 & $5.0986,-21$ & $4.0105,-23$ & $2.9532,-21$ & $2.0142,-23$ & $1.7766,-21$ & $1.0626,-23$ \\
\hline 64 & $5.0986,-21$ & $4.0056,-23$ & $2.9532,-21$ & $2.0117,-23$ & $1.7766,-21$ & $1.0638,-23$ \\
\hline 80 & $5.0986,-21$ & $4.0055,-23$ & $2.9532,-21$ & $2.0118,-23$ & $1.7766,-21$ & $1.0638,-23$ \\
\hline 96 & $5.0986,-21$ & $4.0055,-23$ & $2.9532,-21$ & $2.0118,-23$ & $1.7766,-21$ & $1.0638,-23$ \\
\hline 112 & $5.0986,-21$ & $4.0055,-23$ & $2.9532,-21$ & $2.0118,-23$ & $1.7766,-21$ & $1.0638,-23$ \\
\hline 128 & $5.0986,-21$ & $4.0055,-23$ & $2.9532,-21$ & $2.0118,-23$ & $1.7766,-21$ & $1.0638,-23$ \\
\hline 144 & $5.0986,-21$ & $4.0055,-23$ & $2.9532,-21$ & $2.0118,-23$ & $1.7766,-21$ & $1.0638,-23$ \\
\hline 160 & $5.0986,-21$ & $4.0055,-23$ & $2.9532,-21$ & $2.0118,-23$ & $1.7766,-21$ & $1.0638,-23$ \\
\hline 176 & $5.0986,-21$ & $4.0055,-23$ & $2.9532,-21$ & $2.0118,-23$ & $1.7766,-21$ & $1.0638,-23$ \\
\hline 192 & $5.0986,-21$ & $4.0055,-23$ & $2.9532,-21$ & $2.0118,-23$ & $1.7766,-21$ & $1.0638,-23$ \\
\hline
\end{tabular}


Table 2 continued

\begin{tabular}{|c|c|c|c|c|c|c|}
\hline $\begin{array}{l}\text { Method } \\
E(\mathrm{keV}) \\
N / Q\left(\mathrm{~cm}^{2}\right)\end{array}$ & $\begin{array}{l}\text { CB1 } \\
3250\end{array}$ & $\begin{array}{l}\text { CDW } \\
3250\end{array}$ & $\begin{array}{l}\text { CB1 } \\
3500\end{array}$ & $\begin{array}{l}\text { CDW } \\
3500\end{array}$ & $\begin{array}{l}\text { CB1 } \\
3750\end{array}$ & $\begin{array}{l}\text { CDW } \\
3750\end{array}$ \\
\hline 48 & $1.1046,-21$ & $5.8563,-24$ & $7.0697,-22$ & $3.3611,-24$ & $4.6419,-22$ & $2.0010,-24$ \\
\hline 64 & $1.1046,-21$ & $5.8804,-24$ & $7.0697,-22$ & $3.3756,-24$ & $4.6419,-22$ & $2.0017,-24$ \\
\hline 80 & $1.1046,-21$ & $5.8781,-24$ & $7.0697,-22$ & $3.3742,-24$ & $4.6419,-22$ & $2.0029,-24$ \\
\hline 96 & $1.1046,-21$ & $5.8781,-24$ & $7.0697,-22$ & $3.3744,-24$ & $4.6419,-22$ & $2.0028,-24$ \\
\hline 112 & $1.1046,-21$ & $5.8781,-24$ & $7.0697,-22$ & $3.3744,-24$ & $4.6419,-22$ & $2.0028,-24$ \\
\hline 128 & $1.1046,-21$ & $5.8781,-24$ & $7.0697,-22$ & $3.3744,-24$ & $4.6419,-22$ & $2.0028,-24$ \\
\hline 144 & $1.1046,-21$ & $5.8781,-24$ & $7.0697,-22$ & $3.3744,-24$ & $4.6419,-22$ & $2.0028,-24$ \\
\hline 160 & $1.1046,-21$ & $5.8781,-24$ & $7.0697,-22$ & $3.3744,-24$ & $4.6419,-22$ & $2.0028,-24$ \\
\hline 176 & $1.1046,-21$ & $5.8781,-24$ & $7.0697,-22$ & $3.3744,-24$ & $4.6419,-22$ & $2.0028,-24$ \\
\hline 192 & $1.1046,-21$ & $5.8781,-24$ & $7.0697,-22$ & $3.3744,-24$ & $4.6419,-22$ & $2.0028,-24$ \\
\hline $\begin{array}{l}\text { Method } \\
E(\mathrm{keV}) \\
N / Q\left(\mathrm{~cm}^{2}\right)\end{array}$ & $\begin{array}{l}\text { CB1 } \\
4000\end{array}$ & $\begin{array}{l}\text { CDW } \\
4000\end{array}$ & $\begin{array}{l}\text { CB1 } \\
4250\end{array}$ & $\begin{array}{l}\text { CDW } \\
4250\end{array}$ & $\begin{array}{l}\text { CB1 } \\
4500\end{array}$ & $\begin{array}{l}\text { CDW } \\
4500\end{array}$ \\
\hline 48 & $3.1181,-22$ & $1.2286,-24$ & $2.1379,-22$ & $7.7345,-25$ & $1.4932,-22$ & $4.9699,-25$ \\
\hline 64 & $3.1181,-22$ & $1.2223,-24$ & $2.1379,-22$ & $7.6694,-25$ & $1.4932,-22$ & $4.9348,-25$ \\
\hline 80 & $3.1181,-22$ & $1.2245,-24$ & $2.1379,-22$ & $7.6832,-25$ & $1.4932,-22$ & $4.9341,-25$ \\
\hline 96 & $3.1181,-22$ & $1.2243,-24$ & $2.1379,-22$ & $7.6831,-25$ & $1.4932,-22$ & $4.9365,-25$ \\
\hline 112 & $3.1181,-22$ & $1.2243,-24$ & $2.1379,-22$ & $7.6830,-25$ & $1.4932,-22$ & $4.9360,-25$ \\
\hline 128 & $3.1181,-22$ & $1.2243,-24$ & $2.1379,-22$ & $7.6831,-25$ & $1.4932,-22$ & $4.9361,-25$ \\
\hline 144 & $3.1181,-22$ & $1.2243,-24$ & $2.1379,-22$ & $7.6831,-25$ & $1.4932,-22$ & $4.9361,-25$ \\
\hline 160 & $3.1181,-22$ & $1.2243,-24$ & $2.1379,-22$ & $7.6831,-25$ & $1.4932,-22$ & $4.9360,-25$ \\
\hline 176 & $3.1181,-22$ & $1.2243,-24$ & $2.1379,-22$ & $7.6831,-25$ & $1.4932,-22$ & $4.9361,-25$ \\
\hline 192 & $3.1181,-22$ & $1.2243,-24$ & $2.1379,-22$ & $7.6831,-25$ & $1.4932,-22$ & $4.9361,-25$ \\
\hline $\begin{array}{l}\text { Method } \\
E(\mathrm{keV}) \\
N / Q\left(\mathrm{~cm}^{2}\right)\end{array}$ & $\begin{array}{l}\text { CB1 } \\
4750\end{array}$ & $\begin{array}{l}\text { CDW } \\
4750\end{array}$ & $\begin{array}{l}\text { CB1 } \\
5000\end{array}$ & $\begin{array}{l}\text { CDW } \\
5000\end{array}$ & $\begin{array}{l}\text { CB1 } \\
5500\end{array}$ & $\begin{array}{l}\text { CDW } \\
5500\end{array}$ \\
\hline 48 & $1.0605,-22$ & $3.2513,-25$ & $7.6486,-23$ & $2.1634,-25$ & $4.1442,-23$ & $1.0079,-25$ \\
\hline 64 & $1.0605,-22$ & $3.2463,-25$ & $7.6486,-23$ & $2.1758,-25$ & $4.1442,-23$ & $1.0228,-25$ \\
\hline 80 & $1.0605,-22$ & $3.2372,-25$ & $7.6486,-23$ & $2.1663,-25$ & $4.1442,-23$ & $1.0218,-25$ \\
\hline 96 & $1.0605,-22$ & $3.2392,-25$ & $7.6486,-23$ & $2.1664,-25$ & $4.1442,-23$ & $1.0201,-25$ \\
\hline 112 & $1.0605,-22$ & $3.2391,-25$ & $7.6486,-23$ & $2.1668,-25$ & $4.1442,-23$ & $1.0200,-25$ \\
\hline 128 & $1.0605,-22$ & $3.2390,-25$ & $7.6486,-23$ & $2.1666,-25$ & $4.1442,-23$ & $1.0201,-25$ \\
\hline 144 & $1.0605,-22$ & $3.2390,-25$ & $7.6486,-23$ & $2.1666,-25$ & $4.1442,-23$ & $1.0201,-25$ \\
\hline 160 & $1.0605,-22$ & $3.2390,-25$ & $7.6486,-23$ & $2.1666,-25$ & $4.1442,-23$ & $1.0201,-25$ \\
\hline 176 & $1.0605,-22$ & $3.2390,-25$ & $7.6486,-23$ & $2.1666,-25$ & $4.1442,-23$ & $1.0201,-25$ \\
\hline 192 & $1.0605,-22$ & $3.2390,-25$ & $7.6486,-23$ & $2.1666,-25$ & $4.1442,-23$ & $1.0201,-25$ \\
\hline
\end{tabular}


Table 2 continued

\begin{tabular}{lllllll}
\hline $\begin{array}{l}\text { Method } \\
E(\mathrm{keV})\end{array}$ & CB1 & CDW & CB1 & CDW & CB1 & $\begin{array}{l}\text { CDW } \\
N / Q\left(\mathrm{~cm}^{2}\right)\end{array}$ \\
\hline 48 & 6000 & 6000 & 6500 & 6500 & 7000 & 7000 \\
64 & $2.3548,-23$ & $5.0312,-26$ & $1.3938,-23$ & $2.6724,-26$ & $8.5467,-24$ & $1.4889,-26$ \\
80 & $2.3548,-23$ & $5.0708,-26$ & $1.3938,-23$ & $2.6529,-26$ & $8.5467,-24$ & $1.4618,-26$ \\
96 & $2.3548,-23$ & $5.0971,-26$ & $1.3938,-23$ & $2.6665,-26$ & $8.5467,-24$ & $1.4610,-26$ \\
112 & $2.3548,-23$ & $5.0969,-26$ & $1.3938,-23$ & $2.6740,-26$ & $8.5467,-24$ & $1.4644,-26$ \\
128 & $2.3548,-23$ & $5.0943,-26$ & $1.3938,-23$ & $2.6753,-26$ & $8.5467,-24$ & $1.4661,-26$ \\
144 & $2.3548,-23$ & $5.0939,-26$ & $1.3938,-23$ & $2.6750,-26$ & $8.5467,-24$ & $1.4666,-26$ \\
160 & $2.3548,-23$ & $5.0941,-26$ & $1.3938,-23$ & $2.6748,-26$ & $8.5467,-24$ & $1.4666,-26$ \\
176 & $2.3548,-23$ & $5.0942,-26$ & $1.3938,-23$ & $2.6747,-26$ & $8.5467,-24$ & $1.4666,-26$ \\
192 & $2.3548,-23$ & $5.0942,-26$ & $1.3938,-23$ & $2.6747,-26$ & $8.5467,-24$ & $1.4666,-26$ \\
& $2.3548,-23$ & $5.0942,-26$ & $1.3938,-23$ & $2.6748,-26$ & $8.5467,-24$ & $1.4665,-26$ \\
\hline
\end{tabular}

Therefore, due to the unequal transition amplitudes in the BCIS-4B and BDW4B methods for process (2.1), some differences are expected in the resulting cross sections for these two theories. It would then be of interest to see to which extent the difference between $Q_{\mathrm{BCIS}-4 \mathrm{~B}}$ and $Q_{\mathrm{BDW}-4 \mathrm{~B}}$ can be for process (2.1). This is illustrated in Fig. 4, which makes a direct comparison between $Q_{\mathrm{BCIS}-4 \mathrm{~B}}$ and $Q_{\mathrm{BDW}-4 \mathrm{~B}}$ on the same panels for $N=48,64,80,96,128$ and $N=192$. The difference seen on the six panels (a)-(f) from Fig. 4 is quite small at $E \geq 150 \mathrm{keV}$ (with some oscillations or undulations at $100 \leq E<150 \mathrm{keV}$ for $N=48$ ). This is an appealing feature of the BCIS-4B and BDW-4B methods, especially given that $Q_{\mathrm{BCIS}-4 \mathrm{~B}}$ and $Q_{\mathrm{BDW}-4 \mathrm{~B}}$ are computed with the simplest one-parameter Hyllerras' [95] ground-state wavefunction of helium. Based on this finding, it might be anticipated that even a better agreement between $Q_{\mathrm{BCIS}-4 \mathrm{~B}}$ and $Q_{\mathrm{BDW}-4 \mathrm{~B}}$ could take place with more elaborated heliumlike bound-state wavefunctions (e.g. those with some $\sim 60$ variational parameters from the so-called 'configuration-interaction' formalism [97]).

The CB1-4B and CDW-4B methods are purely the first- and second-order methods, respectively. These are the two extremes in the same formalism. The CB1-method is of a first-order because it invokes no electronic continua whatsoever (centered on either the projectile or target nucleus). By contrast, the CDW-4B method is of a second-order since it takes into account all the electronic continua of the two electrons (on both the projectile and target nucleus in the in the entrance and exit channel, respectively). On the other hand, the asymmetric second-order approximations are also of interest to consider as they make some bridges between the pure first- and second-order methods. In particular, the BCIS-4B and BDW-4B methods are the two different hybridizations of the CDW-4B and CB1-4B methods. They take the formalism of the CDW-4B method in one channel and that of the CB1-4B method in the other channel. As such, the BCIS-4B and BDW-4B methods include the one-center electronic continua on only one nucleus (either of the projectile or target nucleus). In other words, as opposed to the two-center electronic continua in the CDW-4B method, the one-center electronic 
Table 3 Total cross sections $Q\left(\mathrm{~cm}^{2}\right)$ as a function of impact energy $E(\mathrm{keV})$ for double charge exchange ${ }^{4} \mathrm{He}^{2+}+{ }^{4} \mathrm{He}\left({ }^{1} \mathrm{~S}\right) \rightarrow{ }^{4} \mathrm{He}\left({ }^{1} \mathrm{~S}\right)+{ }^{4} \mathrm{He}^{2+}$ using the Gauss-Legendre quadrature set with order $N=192$ per each of three-to-five-dimensional (3D-5D) numerical integrations in the BCIS-4B (5D), BDW-4B (5D), CB1-4B (3D) and CDW-4B (4D) methods. Notation $2.3431,-17$ means $2.3431 \times 10^{-17} \mathrm{~cm}^{2}$

\begin{tabular}{|c|c|c|c|c|}
\hline $\begin{array}{l}\text { Method } \\
E(\mathrm{keV}) / Q\left(\mathrm{~cm}^{2}\right)\end{array}$ & BCIS & BDW & CB1 & CDW \\
\hline 100 & $2.3431,-17$ & $1.7626,-17$ & $3.5898,-16$ & $5.3139,-16$ \\
\hline 110 & $2.4821,-17$ & $1.8659,-17$ & $3.0486,-16$ & $3.8247,-16$ \\
\hline 117.5 & $2.5594,-17$ & $1.9154,-17$ & $2.7132,-16$ & $3.0380,-16$ \\
\hline 125 & $2.6023,-17$ & $1.9390,-17$ & $2.4256,-16$ & $2.4425,-16$ \\
\hline 137.5 & $2.5992,-17$ & $1.9287,-17$ & $2.0299,-16$ & $1.7375,-16$ \\
\hline 150 & $2.5258,-17$ & $1.8736,-17$ & $1.7148,-16$ & $1.2668,-16$ \\
\hline 162.5 & $2.4098,-17$ & $1.7907,-17$ & $1.4603,-16$ & $9.4296,-17$ \\
\hline 175 & $2.2715,-17$ & $1.6922,-17$ & $1.2523,-16$ & $7.1438,-17$ \\
\hline 187.5 & $2.1236,-17$ & $1.5861,-17$ & $1.0805,-16$ & $5.4952,-17$ \\
\hline 200 & $1.9735,-17$ & $1.4774,-17$ & $9.3737,-17$ & $4.2838,-17$ \\
\hline 225 & $1.6835,-17$ & $1.2648,-17$ & $7.1556,-17$ & $2.6932,-17$ \\
\hline 250 & $1.4204,-17$ & $1.0698,-17$ & $5.5513,-17$ & $1.7582,-17$ \\
\hline 300 & $9.9278,-18$ & $7.5062,-18$ & $3.4738,-17$ & $8.1755,-18$ \\
\hline 350 & $6.8720,-18$ & $5.2154,-18$ & $2.2653,-17$ & $4.1526,-18$ \\
\hline 400 & $4.7580,-18$ & $3.6265,-18$ & $1.5263,-17$ & $2.2542,-18$ \\
\hline 450 & $3.3122,-18$ & $2.5367,-18$ & $1.0563,-17$ & $1.2894,-18$ \\
\hline 500 & $2.3242,-18$ & $1.7892,-18$ & $7.4774,-18$ & $7.6957,-19$ \\
\hline 550 & $1.6458,-18$ & $1.2739,-18$ & $5.3971,-18$ & $4.7593,-19$ \\
\hline 600 & $1.1767,-18$ & $9.1591,-19$ & $3.9624,-18$ & $3.0336,-19$ \\
\hline 625 & $9.9852,-19$ & $7.7948,-19$ & $3.4146,-18$ & $2.4464,-19$ \\
\hline 650 & $8.4935,-19$ & $6.6496,-19$ & $2.9531,-18$ & $1.9849,-19$ \\
\hline 700 & $6.1885,-19$ & $4.8737,-19$ & $2.2306,-18$ & $1.3288,-19$ \\
\hline 750 & $4.5502,-19$ & $3.6050,-19$ & $1.7053,-18$ & $9.0780,-20$ \\
\hline 800 & $3.3748,-19$ & $2.6901,-19$ & $1.3181,-18$ & $6.3153,-20$ \\
\hline 825 & $2.9156,-19$ & $2.3312,-19$ & $1.1632,-18$ & $5.3003,-20$ \\
\hline 850 & $2.5239,-19$ & $2.0243,-19$ & $1.0290,-18$ & $4.4658,-20$ \\
\hline 900 & $1.9026,-19$ & $1.5355,-19$ & $8.1066,-19$ & $3.2050,-20$ \\
\hline 950 & $1.4452,-19$ & $1.1736,-19$ & $6.4406,-19$ & $2.3314,-20$ \\
\hline 1000 & $1.1056,-19$ & $9.0353,-20$ & $5.1568,-19$ & $1.7170,-20$ \\
\hline 1250 & $3.1909,-20$ & $2.6928,-20$ & $1.8732,-19$ & $4.3433,-21$ \\
\hline 1500 & $1.0549,-20$ & $9.1982,-21$ & $7.7542,-20$ & $1.3425,-21$ \\
\hline 1750 & $3.8905,-21$ & $3.5054,-21$ & $3.5428,-20$ & $4.8096,-22$ \\
\hline 2000 & $1.5695,-21$ & $1.4611,-21$ & $1.7495,-20$ & $1.9301,-22$ \\
\hline 2250 & $6.8248,-22$ & $6.5619,-22$ & $9.2007,-21$ & $8.4761,-23$ \\
\hline 2500 & $3.1631,-22$ & $3.1388,-22$ & $5.0986,-21$ & $4.0055,-23$ \\
\hline 2750 & $1.5488,-22$ & $1.5847,-22$ & $2.9532,-21$ & $2.0118,-23$ \\
\hline
\end{tabular}


Table 3 continued

\begin{tabular}{lllll}
\hline $\begin{array}{l}\text { Method } \\
E(\mathrm{keV}) / Q\left(\mathrm{~cm}^{2}\right)\end{array}$ & BCIS & BDW & CB1 & CDW \\
\hline 3000 & $7.9564,-23$ & $8.3832,-23$ & $1.7766,-21$ & $1.0638,-23$ \\
3250 & $4.2639,-23$ & $4.6197,-23$ & $1.1046,-21$ & $5.8781,-24$ \\
3500 & $2.3727,-23$ & $2.6389,-23$ & $7.0697,-22$ & $3.3744,-24$ \\
3750 & $1.3659,-23$ & $1.5564,-23$ & $4.6419,-22$ & $2.0028,-24$ \\
4000 & $8.1083,-24$ & $9.4445,-24$ & $3.1181,-22$ & $1.2243,-24$ \\
4250 & $4.9507,-24$ & $5.8799,-24$ & $2.1379,-22$ & $7.6831,-25$ \\
4500 & $3.1019,-24$ & $3.7465,-24$ & $1.4932,-22$ & $4.9361,-25$ \\
4750 & $1.9907,-24$ & $2.4378,-24$ & $1.0605,-22$ & $3.2390,-25$ \\
5000 & $1.3064,-24$ & $1.6169,-24$ & $7.6486,-23$ & $2.1666,-25$ \\
5500 & $5.9813,-25$ & $7.4867,-25$ & $4.1442,-23$ & $1.0201,-25$ \\
6000 & $2.9484,-25$ & $3.6792,-25$ & $2.3548,-23$ & $5.0942,-26$ \\
6500 & $1.5520,-25$ & $1.9027,-25$ & $1.3938,-23$ & $2.6748,-26$ \\
7000 & $8.6628,-26$ & $1.0284,-25$ & $8.5467,-24$ & $1.4665,-26$ \\
7500 & $5.0939,-26$ & $5.7780,-26$ & $5.4058,-24$ & $8.3503,-27$ \\
8000 & $3.1367,-26$ & $3.3589,-26$ & $3.5139,-24$ & $4.9151,-27$ \\
8500 & $2.0115,-26$ & $2.0127,-26$ & $2.3404,-24$ & $2.9798,-27$ \\
9000 & $1.3366,-26$ & $1.2391,-26$ & $1.5930,-24$ & $1.8546,-27$ \\
9500 & $9.1608,-27$ & $7.8171,-27$ & $1.1058,-24$ & $1.1819,-27$ \\
10000 & $6.4497,-27$ & $5.0414,-27$ & $7.8126,-25$ & \\
\hline & & & & \\
& & & & \\
& & & & \\
\hline
\end{tabular}

continua are encountered in the BCIS-4B and BDW-4B methods. As to the perturbation potentials in the transition amplitudes, they are the same in the BDW-4B and CDW4B methods. Likewise, the perturbation potentials in the transition amplitudes of the BCIS-4B and CB1-4B methods are identical.

There is also the CDW-EIS-4B method which is an asymmetrically eikonalized version of the CDW-4B method. In the CDW-EIS-4B method, the two electronic Coulomb wavefunctions from the CDW-4B method in the entrance channel are replaced by their asymptotic forms. However, these latter forms are valid only at infinitely large separations of the projectile nucleus from the two target electrons. In the exit channel, the CDW-EIS-4B method uses the full Coulomb wavefunctions of the two electrons as in the CDW-4B method. Comparisons between the CDW-4B and CDW-EIS-4B method would make in evidence the validity of additionally approximating the CDW4B method by eikonalizing the double electronic continua in the entrance channel.

Also important is to compare $Q_{\mathrm{CDW}-\mathrm{EIS}-4 \mathrm{~B}}$ with $Q_{\mathrm{BCIS}-4 \mathrm{~B}}$ and $Q_{\mathrm{BDW}-4 \mathrm{~B}}$. The key difference among these three cross sections is in using the logarithmic Coulomb phase factors. In $Q_{\mathrm{CDW}-\mathrm{EIS}-4 \mathrm{~B}}$, these asymptotic phases depend on distance of the projectile nucleus from the target electrons. By contrast, in $Q_{\mathrm{BCIS}-4 \mathrm{~B}}$ and $Q_{\mathrm{BDW}-4 \mathrm{~B}}$, the Coulomb phases depend on the nucleus-nucleus separation. This difference is immaterial at infinitely large distances when all the phases are considered outside the integrals in the transition amplitudes. However, the integrals are over all dis- 
Convergence of Cross Sections for Two-Electron Transfer $(\alpha-\mathrm{He})$ : BCIS-4B vs BDW-4B

Orders $\mathrm{N}=48,64,80$ (vs 192) Per Axis in 5-Dimensional Gauss-Legendre Quadratures

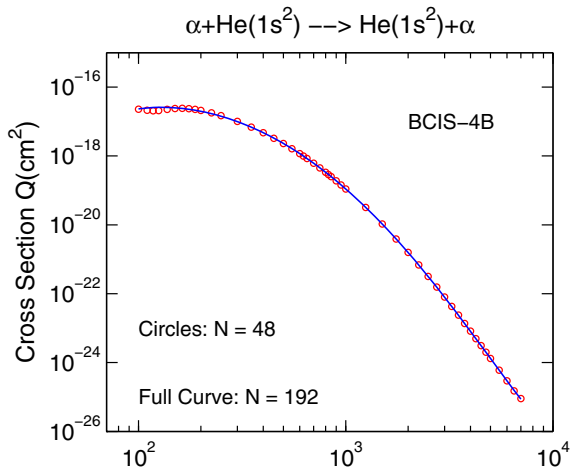

(a) Impact Energy $\mathrm{E}(\mathrm{keV})$

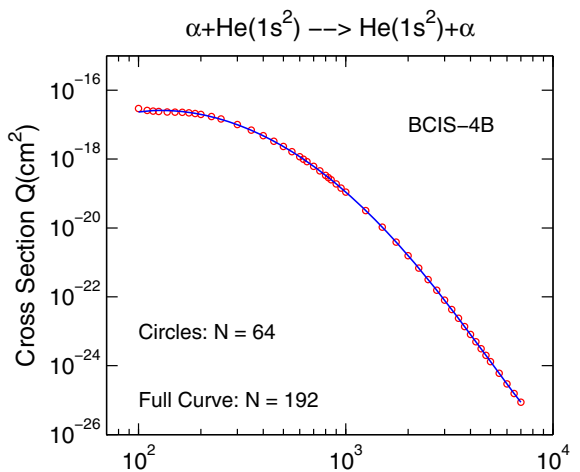

(b) Impact Energy $\mathrm{E}(\mathrm{keV})$

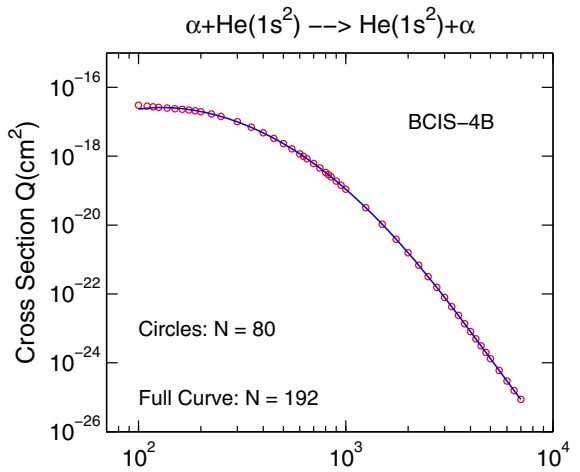

(c) Impact Energy $\mathrm{E}(\mathrm{keV})$

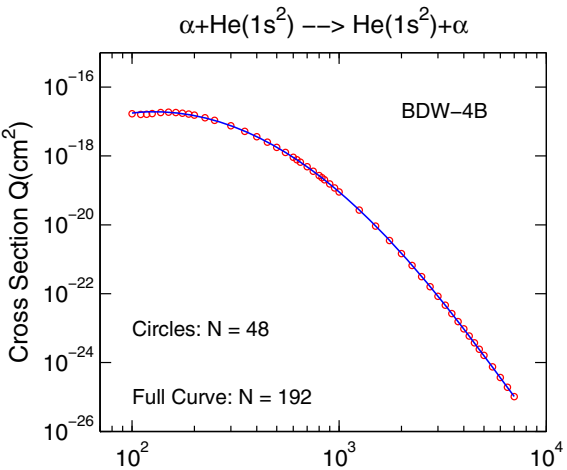

(d) Impact Energy $\mathrm{E}(\mathrm{keV})$

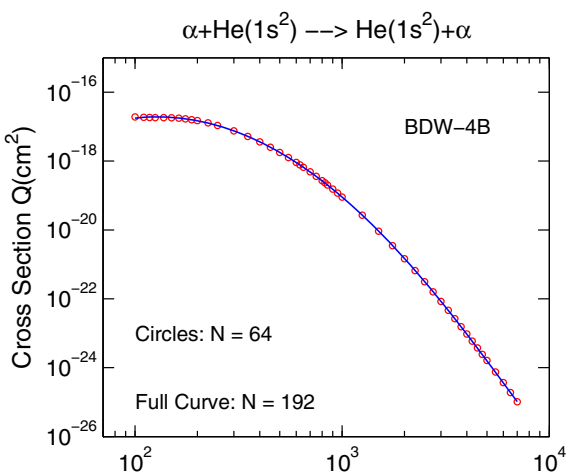

(e) Impact Energy $\mathrm{E}(\mathrm{keV})$

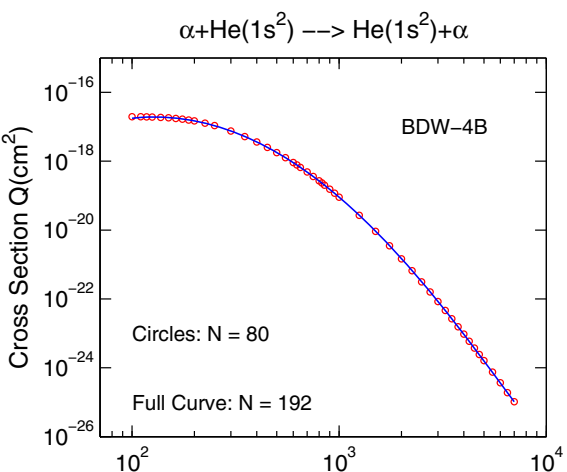

(f) Impact Energy $\mathrm{E}(\mathrm{keV})$

Fig. 1 Total cross sections for double charge exchange (3.1) using a 4 Gauss-Legendre quadrature set with orders $N$ per each of five numerical integrations in the BCIS-4B (left) and BDW-4B (right) methods. Circles: $N=48(\mathbf{a}, \mathbf{d}), 64(\mathbf{b}, \mathbf{e}), 80(\mathbf{c}, \mathbf{f})$. The full curves on each panel are for the converged (or reference) cross sections with $N=192$ (color online) 
Convergence of Cross Sections for Two-Electron Transfer $(\alpha-\mathrm{He})$ : BCIS-4B vs BDW-4B

Orders $\mathrm{N}=96,112,128$ (vs 192) Per Axis in 5-Dimensional Gauss-Legendre Quadratures

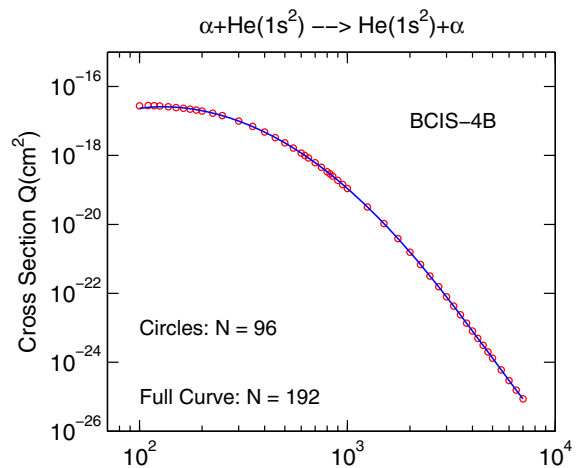

(a) Impact Energy $\mathrm{E}(\mathrm{keV})$

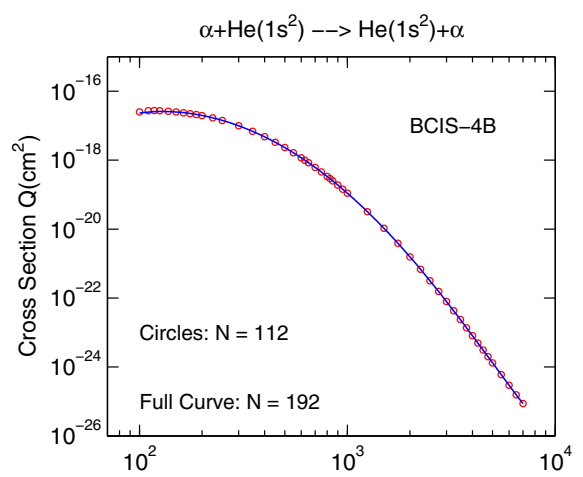

(b) Impact Energy $\mathrm{E}(\mathrm{keV})$

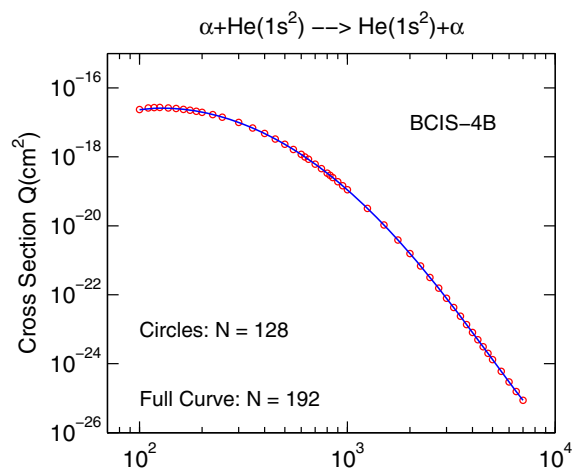

(c) Impact Energy $\mathrm{E}(\mathrm{keV})$

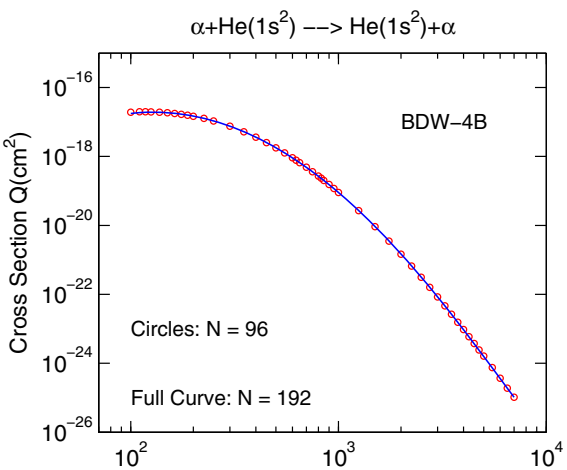

(d) Impact Energy $\mathrm{E}(\mathrm{keV})$

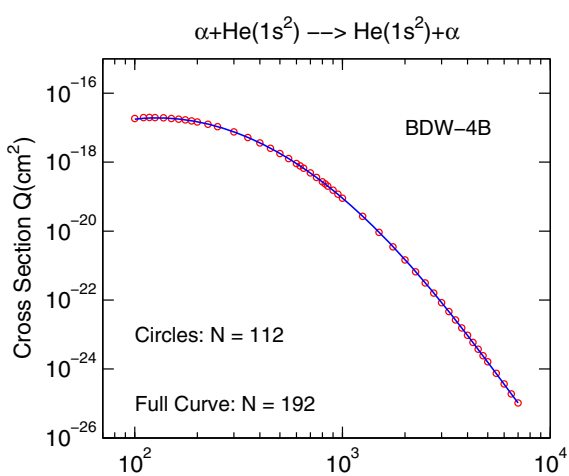

(e) Impact Energy $\mathrm{E}(\mathrm{keV})$

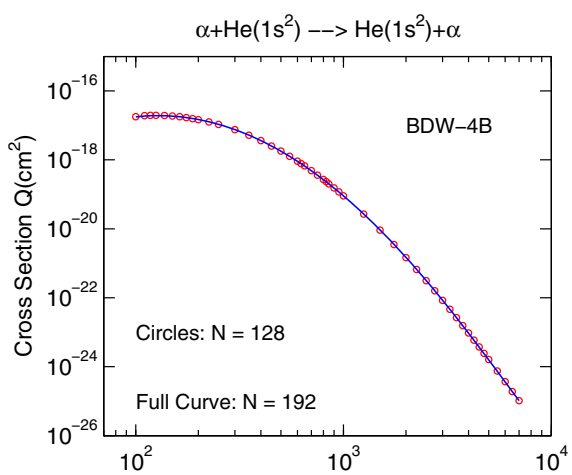

(f) Impact Energy $\mathrm{E}(\mathrm{keV})$

Fig. 2 Total cross sections for double charge exchange (3.1) using a 4 Gauss-Legendre quadrature set with orders $N$ per each of five numerical integrations in the BCIS-4B (left) and BDW-4B (right) methods. Circles: $N=96(\mathbf{a}, \mathbf{d}), 112(\mathbf{b}, \mathbf{e}), 128(\mathbf{c}, \mathbf{f})$. The full curves on each panel are for the converged (or reference) cross sections with $N=192$ (color online) 
Convergence of Cross Sections for Two-Electron Transfer $(\alpha-\mathrm{He})$ : BCIS-4B vs BDW-4B

Orders $\mathrm{N}=144,160,176$ (vs 192) Per Axis in 5-Dimensional Gauss-Legendre Quadratures

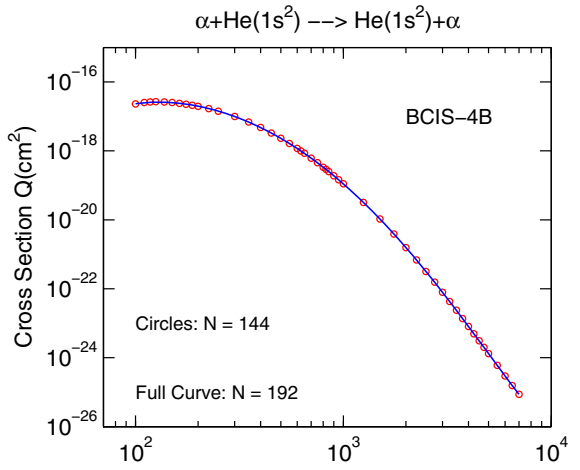

(a) Impact Energy $\mathrm{E}(\mathrm{keV})$

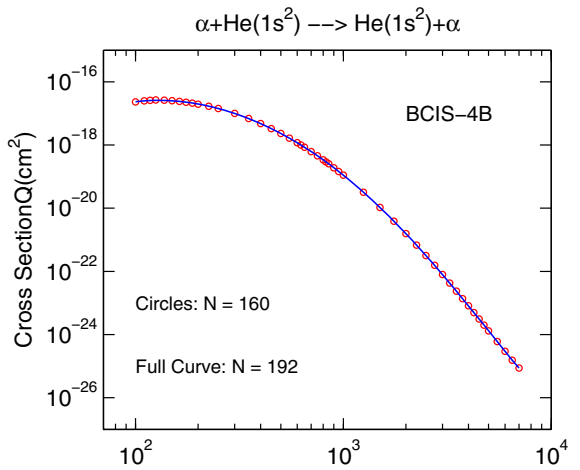

(b) Impact Energy $\mathrm{E}(\mathrm{keV})$

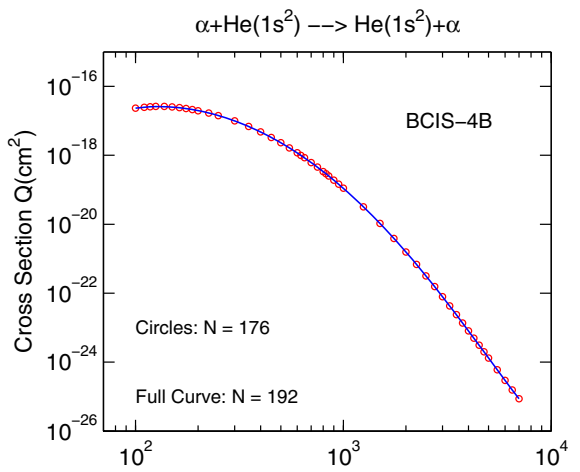

(c) Impact Energy $\mathrm{E}(\mathrm{keV})$

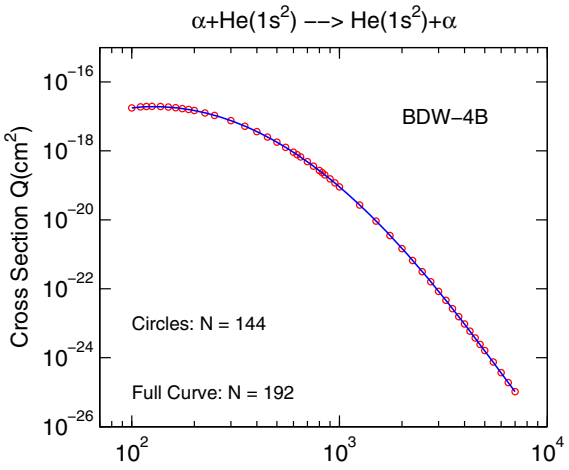

(d) Impact Energy $\mathrm{E}(\mathrm{keV})$

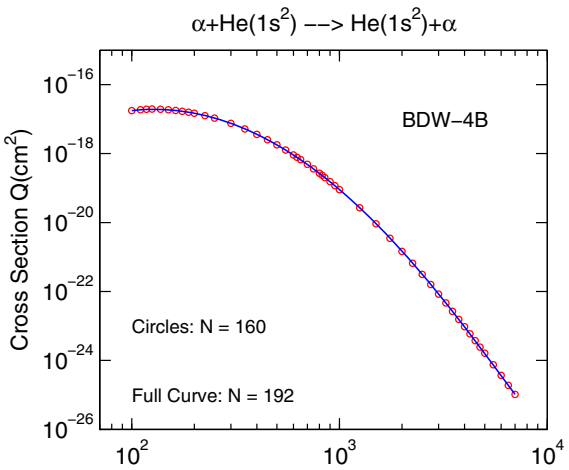

(e) Impact Energy $\mathrm{E}(\mathrm{keV})$

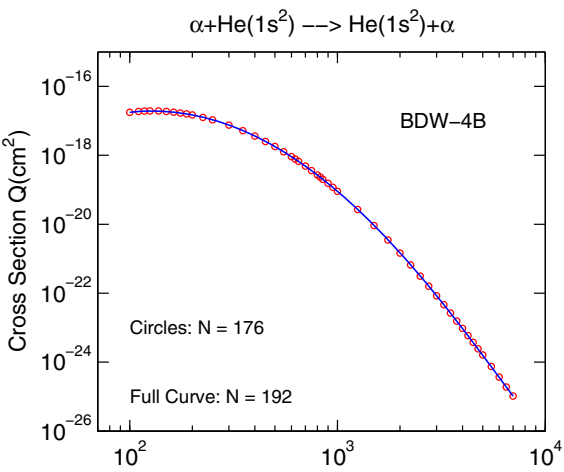

(f) Impact Energy $\mathrm{E}(\mathrm{keV})$

Fig. 3 Total cross sections for double charge exchange (3.1) using a 4 Gauss-Legendre quadrature set with orders $N$ per each of five numerical integrations in the BCIS-4B (left) and BDW-4B (right) methods. Circles: $N=144(\mathbf{a}, \mathbf{d}), 160(\mathbf{b}, \mathbf{e}), 176(\mathbf{c}, \mathbf{f})$. The full curves on each panel are for the converged (or reference) cross sections with $N=192$ (color online) 
Convergence of Cross Sections for Two-Electron Transfer $(\alpha-\mathrm{He})$ : BCIS-4B vs BDW-4B

Orders $\mathrm{N}=48,64,80,96,128,192$ Per Axis in 5-Dimensional Gauss-Legendre Quadratures

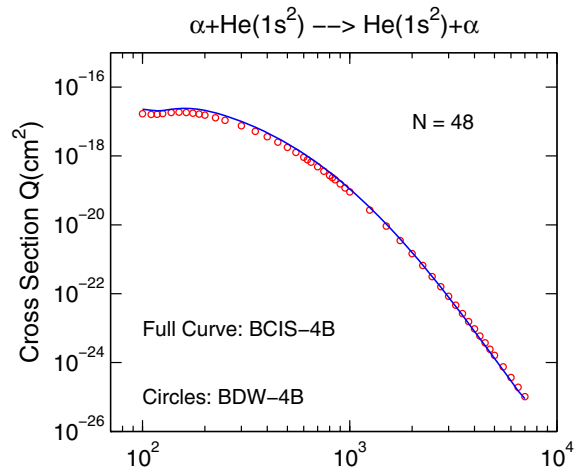

(a) Impact Energy $\mathrm{E}(\mathrm{keV})$

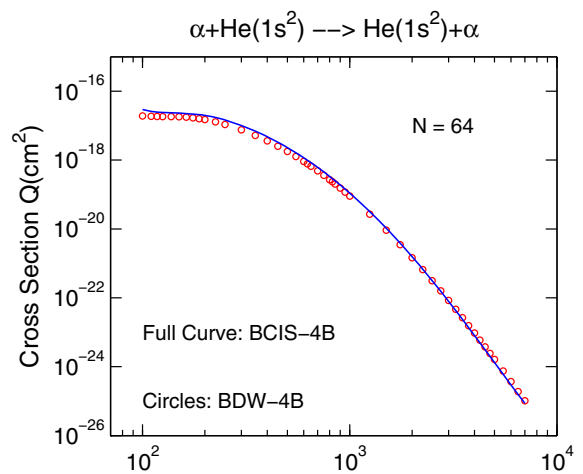

(b) Impact Energy $\mathrm{E}(\mathrm{keV})$

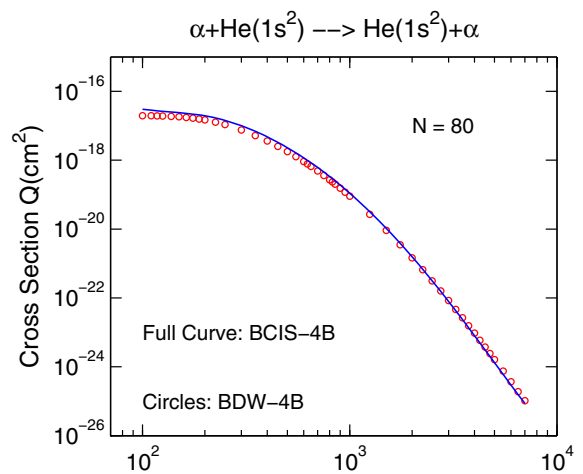

(c) Impact Energy $\mathrm{E}(\mathrm{keV})$

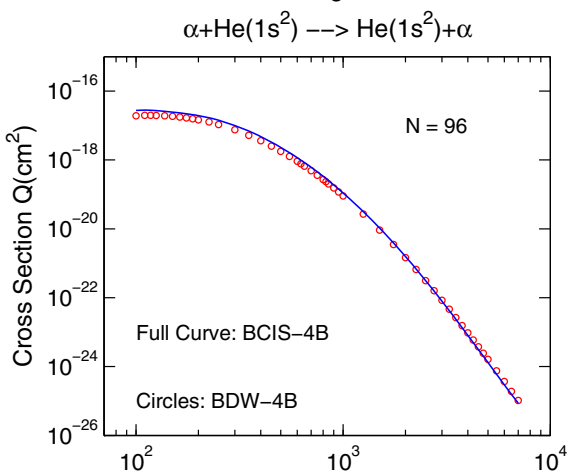

(d) Impact Energy $\mathrm{E}(\mathrm{keV})$

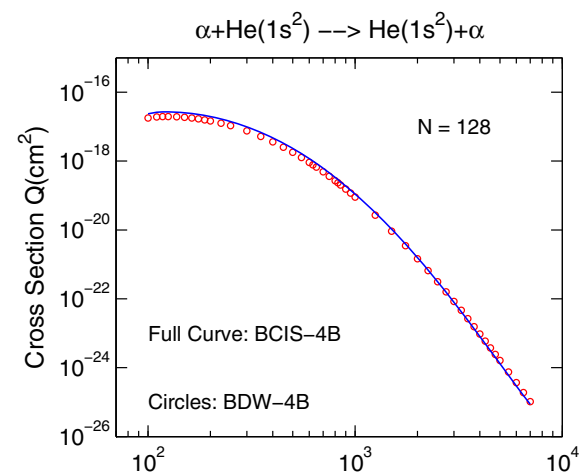

(e) Impact Energy $\mathrm{E}(\mathrm{keV})$

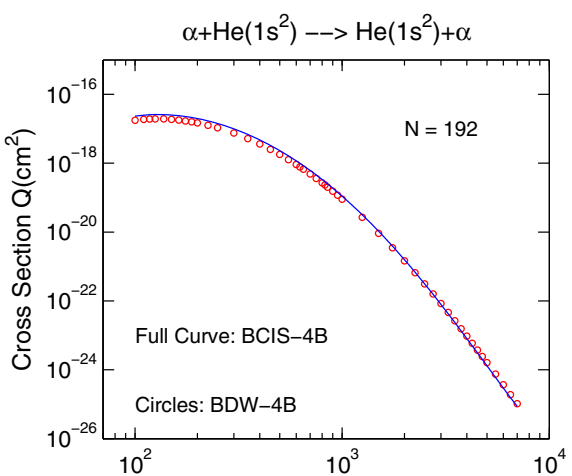

(f) Impact Energy $\mathrm{E}(\mathrm{keV})$

Fig. 4 Total cross sections for double charge exchange (3.1), using a 4 Gauss-Legendre quadrature set with orders $N$ per each of five numerical integrations in the BCIS-4B (full curves) and BDW-4B (circles) methods: $N=48$ (a), 64 (b), $80(\mathbf{c}), 96$ (d), 128 (e), 192 (f). The two methods are compared on the same panels (color online) 
tances (from zero to infinity) Moreover, the dominant contributions to these integrals come from short electron-nucleus distances due to the invoked exponentially decaying bound-state orbitals.

This juxtaposition makes it clear that the physics of these five methods is substantially different. The number of the full (i.e. non-eikonalized) electronic continuum intermediate states is four in the CDW-4B method compared to only two in the BCIS$4 \mathrm{~B}$ and BDW-4B methods. Therefore, comparisons of $Q_{\mathrm{CDW}-4 \mathrm{~B}}$ with $Q_{\mathrm{BCIS}-4 \mathrm{~B}}$ and $Q_{\mathrm{BDW}-4 \mathrm{~B}}$ would permit an assessment of the extent of the influence of the fourfold relative to the twofold electronic continua. Likewise, comparisons of $Q_{\mathrm{CB} 1-4 \mathrm{~B}}$ with $Q_{\mathrm{BCIS}-4 \mathrm{~B}}$ and $Q_{\mathrm{BDW}-4 \mathrm{~B}}$ would allow an estimation of the role of two-electron continuum states in the BCIS-4B and BDW-4B methods with respect to the CB1-4B method where such states are omitted altogether from the onset. The insight from such comparisons can be gained by inspecting Figs. 5, 6, 7 and 8, especially by reference to the available experimental data that are also shown.

Figure 5 plots $Q_{\mathrm{CB} 1-4 \mathrm{~B}}, Q_{\mathrm{BCIS}-4 \mathrm{~B}}, Q_{\mathrm{CDW}-4 \mathrm{~B}}$ and $Q_{\mathrm{CDW}-\mathrm{EIS}-4 \mathrm{~B}}$ alongside the existing experimental data. The CB1-4B method is in a fair agreement with experimental data, but only in a limited interval of the impact energy, $E \in[150,800] \mathrm{keV}$. Above $800 \mathrm{keV}, Q_{\mathrm{CB} 1-4 \mathrm{~B}}$ begins to significantly overestimate the measured cross sections. Such overestimations attain a factor of $\sim 50$ at $3000 \mathrm{keV}$. This latter factor is further augmented with the increased values of $E$. In sharp contrast, at e.g. $E=3000$, it is seen that $Q_{\text {BCIS-4B }}$ is in a perfect accord with the experimental data. This proves that double electronic continuum intermediate states are of great importance for twoelectron transfer processes, especially at high energies. The results for $Q_{\mathrm{BCIS}-4 \mathrm{~B}}$ are plotted in Fig. 5 at $N=48$ and 192 with the highly concordant two curves. Some slight oscillations/undulations are seen at the lowest impact energies $E \in[100,150]$ $\mathrm{keV}$. However, this is inessential as all the shown theoretical cross sections stem from high-energy methods. It has empirically been established in Ref. [3] that the groundto-ground state cross section $Q_{\mathrm{CDW}-3 \mathrm{~B}}$ for single charge exchange (3.3) should be adequate at $E \geq 300 \mathrm{keV}$ for the ${ }^{4} \mathrm{He}^{2+}-{ }^{4} \mathrm{He}^{+}(1 s)$ collisions involving three particles. Extending this estimate to double charge exchange (2.1), it might be assumed that high-energy methods for these four-body collisions should be valid at $E \geq 600$ $\mathrm{keV}$. This empirical estimate is observed in Fig. 5 to be approximately satisfactory for the BCIS-4B method. However, this is not the case at all with either the CDW-4B or the CDW-EIS-4B methods.

The situation is actually worst with the CDW-EIS-4B method which fails flagrantly at all energies $E \in[100,7000]$ at which the experimental data are available. Well within its applicability domain, as far as $E$ is concerned, the total cross sections in the CDW-EIS-4B methods underestimate those in the CDW-4B method by orders of magnitude. This completely invalidates the eikonalization of the double electronic continua from the CDW-EIS-4B method. As such, the CDW-EIS-4B method can be totally discarded from any useful application to double charge exchange processes. All the theories in Fig. 5 take into account only the final ground state of helium. An inclusion of helium singly- and doubly-excited states in the exit channel [55] changes only very slightly e.g. $Q_{\mathrm{CDW}-4 \mathrm{~B}}$ from Fig. 5 . The sole experimental data with which $Q_{\mathrm{CDW}-4 \mathrm{~B}}$ agrees in Fig. 5 are the two cross sections measured by Schuch et al. [87] at 
Double Electron Capture by Alpha Particles from Helium: Theories vs. Measurements

Four-Body Methods (CB1-4B, BCIS-4B, BDW-4B, CDW-4B and CDW-EIS-4B)

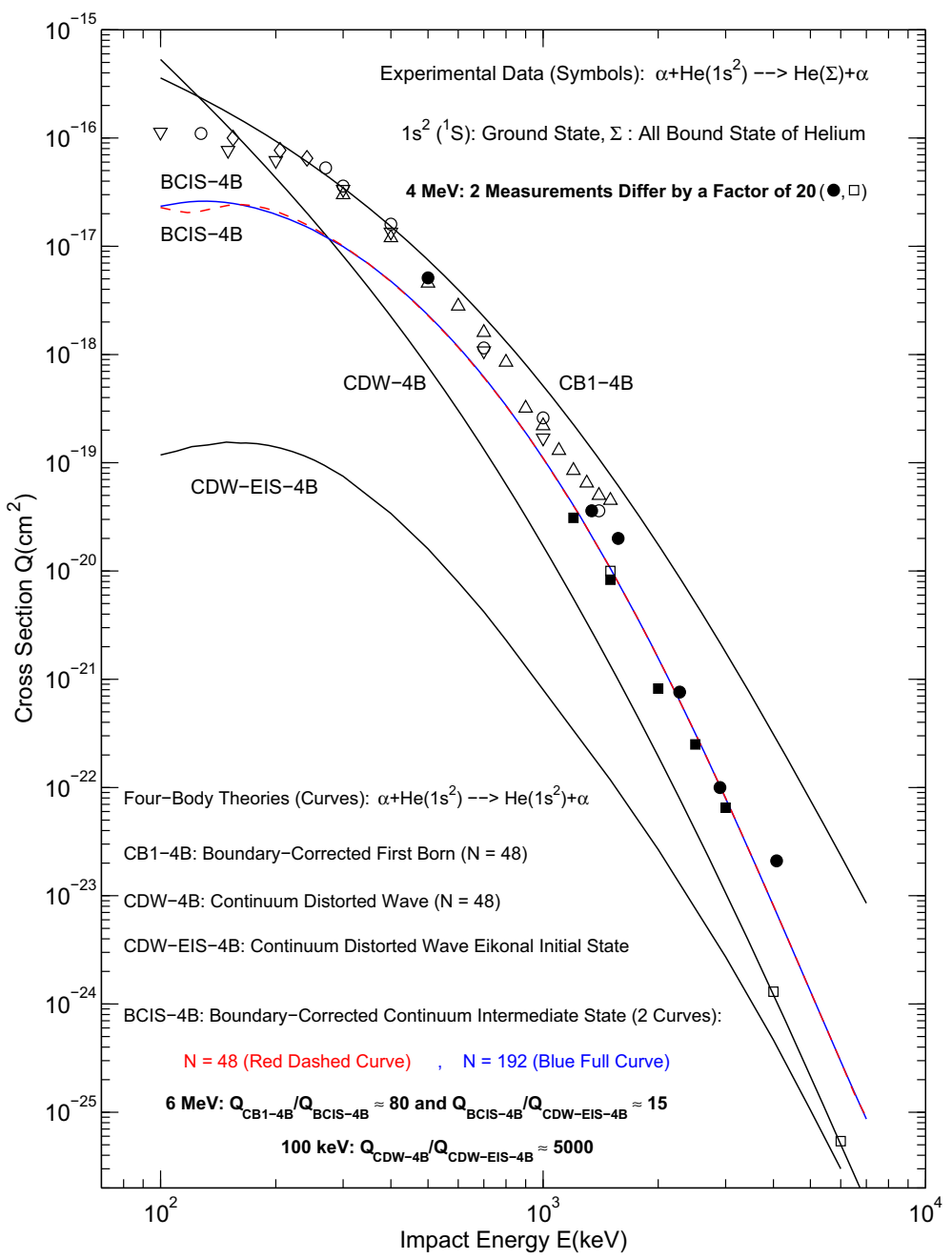

Fig. 5 Total cross sections $Q\left(\mathrm{~cm}^{2}\right)$ as a function of the incident energy $E(\mathrm{keV})$ for two-electron capture from helium by alpha particles. Theories for process (3.1). Measurements for process (3.2). All the theories use the same one-parameter ground-state Hylleraas' wave function [95]. The present computations in the CB14B and CDW-4B methods are for the order $N=48$ per integration axis in the Gauss-Legendre quadrature, whereas 2 values of $N$ are used for the BCIS-4B method: $N=48$ (dashed red curve) and $N=192$ (full blue curve). The results of the CDW-EIS-4B method are from Ref. [55]. Experimental data: $\Delta$ [66], $\diamond$ [69], $\bigcirc$ [77], $\nabla$ [83], $\mathbf{\square}[84], \square[87]$ and $\bullet$ [88] (color online) 
Double Electron Capture by Alpha Particles from Helium: Theories vs. Measurements

Four-Body Methods (CB1-4B, BCIS-4B, BDW-4B, CDW-4B and CDW-EIS-4B)

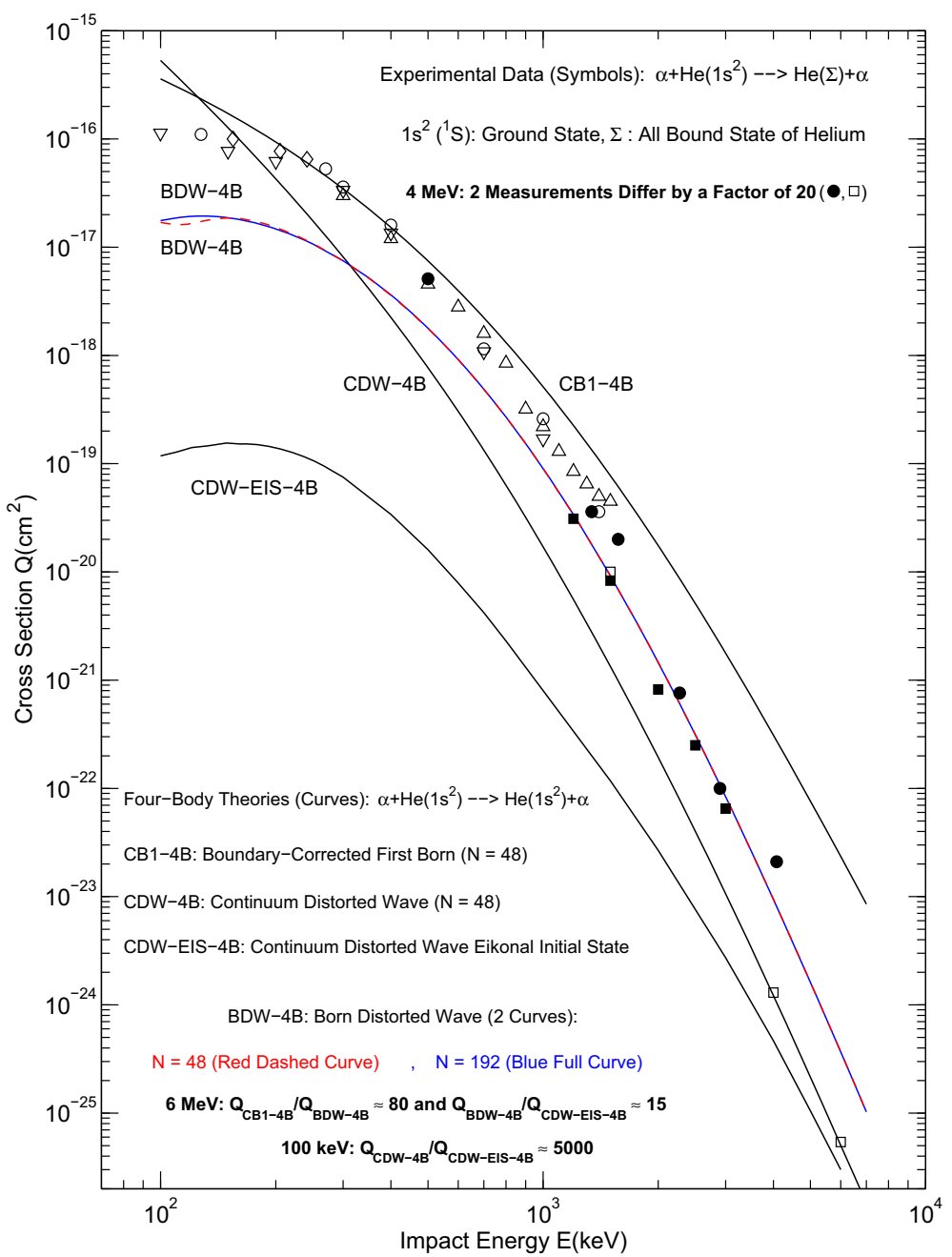

Fig. 6 Total cross sections $Q\left(\mathrm{~cm}^{2}\right)$ as a function of the incident energy $E(\mathrm{keV})$ for two-electron capture from helium by alpha particles. Theories for process (3.1): ground-to-ground-state transition alone. Measurements for process (3.2): ground-to-any-bound-state transitions. All the theories use the same one-parameter ground-state Hylleraas' wave function [95]. The present computations in the CB1-4B and CDW-4B methods are for the order $N=48$ per integration axis in the Gauss-Legendre quadrature, whereas 2 values of $N$ are used for the BDW-4B method: $N=48$ (dashed red curve) and $N=192$ (full blue curve). The results of the CDW-EIS-4B method are from Ref. [55]. Experimental data: $\Delta$ [66], $\diamond$ [69], $\bigcirc$ [77], $\nabla$ [83], $\mathbf{\square}$ [84], $\square[87]$ and $\bullet$ [88] (color online) 
Double Electron Capture by Alpha Particles from Helium: Theories vs. Measurements

Four-Body Methods (CB1-4B, BCIS-4B, BDW-4B, CDW-4B and CDW-EIS-4B)

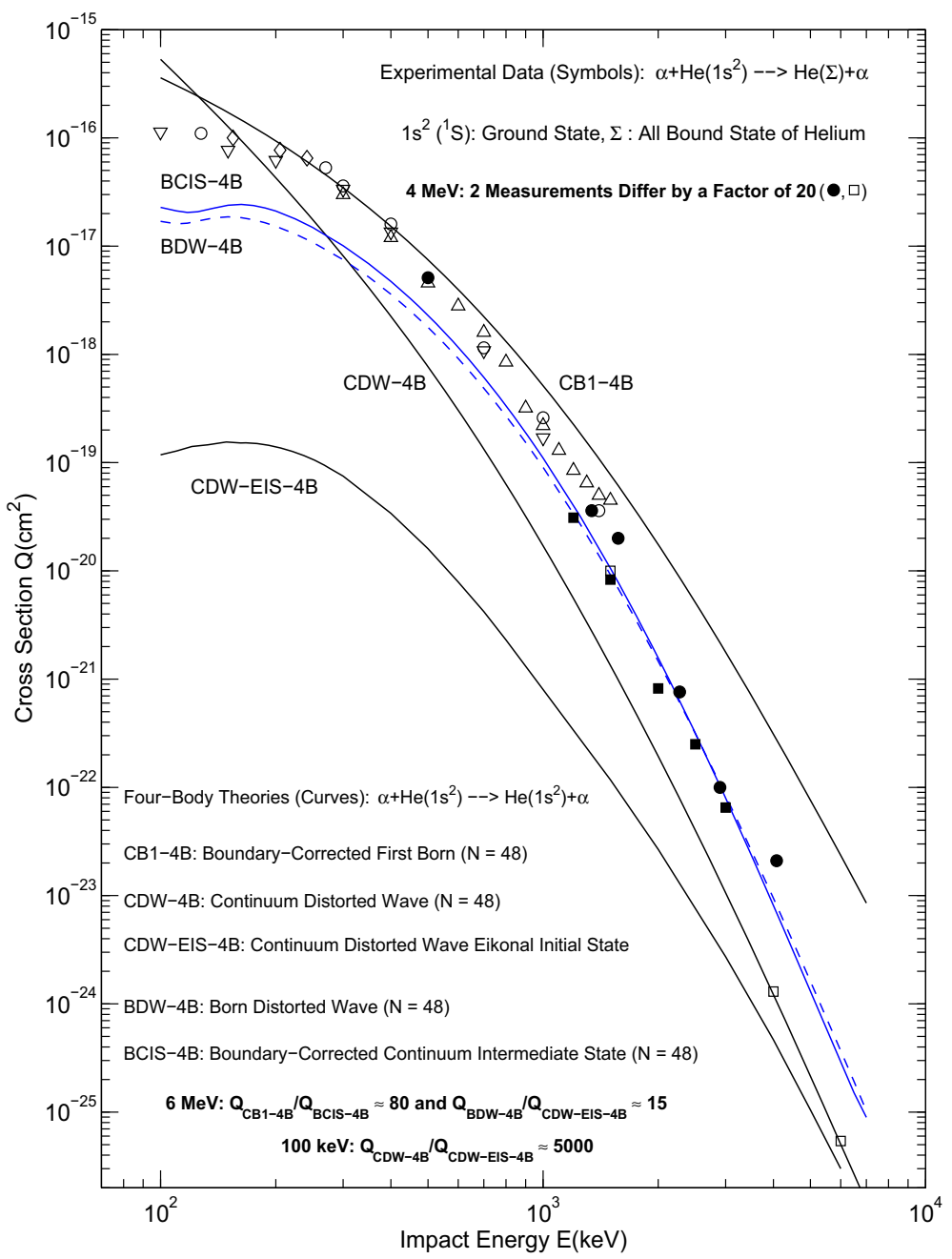

Fig. 7 Total cross sections $Q\left(\mathrm{~cm}^{2}\right)$ as a function of the incident energy $E(\mathrm{keV})$ for two-electron capture from helium by alpha particles. Theories for process (3.1): ground-to-ground-state transition alone. Measurements for process (3.2): ground-to-any-bound-state transitions. All the theories use the same one-parameter ground-state Hylleraas' wave function [95]. The present computations in the CB1-4B (full black), BCIS-4B (full blue), BDW-4B (dashed blue) and CDW-4B (full black) methods are all for the order $N=48$ per integration axis in the Gauss-Legendre quadrature. The results of the CDW-EIS-4B method are from Ref. [55]. Experimental data: $\Delta$ [66], $\diamond[69], \bigcirc[77], \nabla[83], \mathbf{\square}$ [84], $\square$ [87] and $\bullet$ [88] (color online) 
Double Electron Capture by Alpha Particles from Helium: Theories vs. Measurements

Four-Body Methods (CB1-4B, BCIS-4B, BDW-4B, CDW-4B and CDW-EIS-4B)

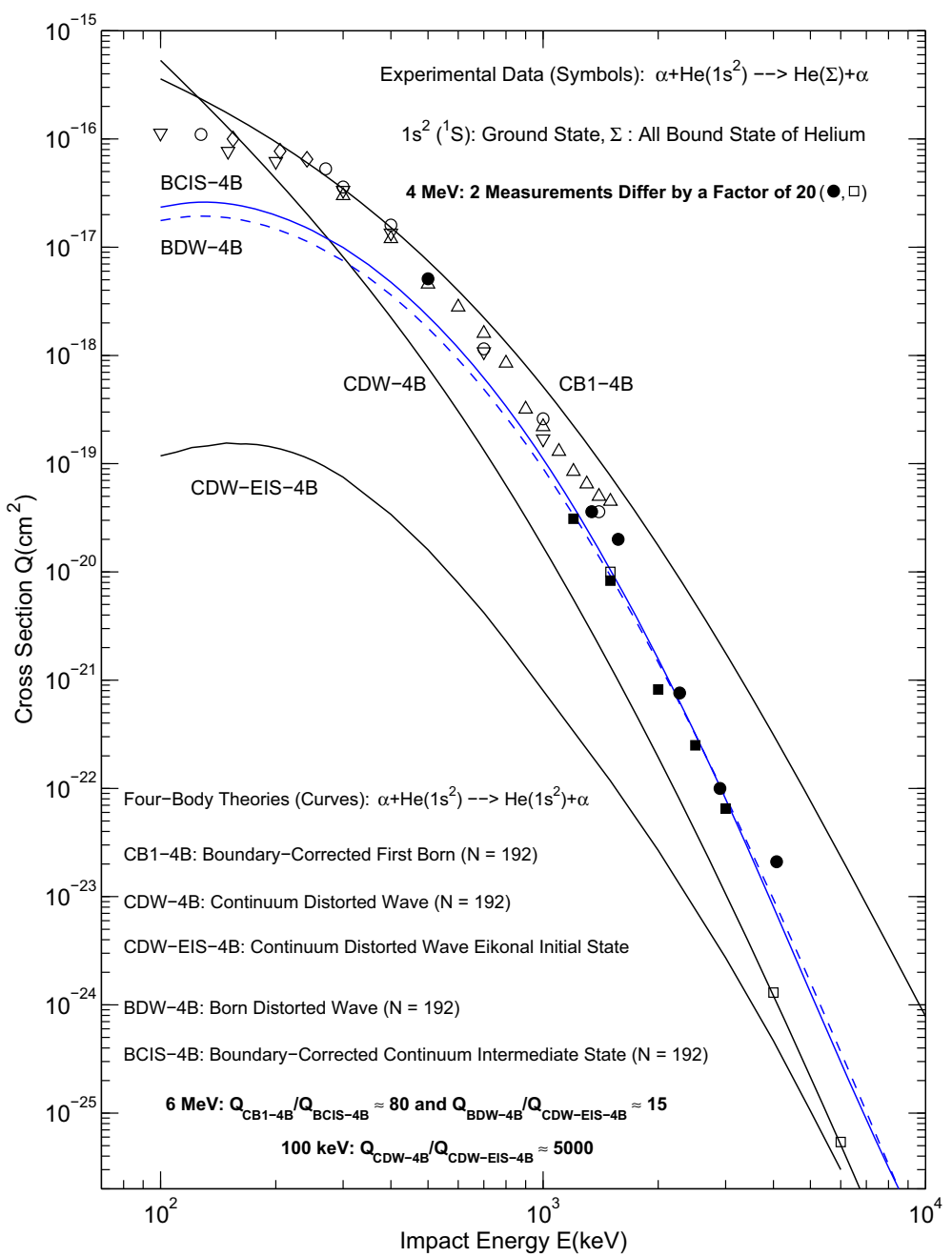

Fig. 8 Total cross sections $Q\left(\mathrm{~cm}^{2}\right)$ as a function of the incident energy $E(\mathrm{keV})$ for two-electron capture from helium by alpha particles. Theories for process (3.1): ground-to-ground-state transition alone. Measurements for process (3.2): ground-to-any-bound-state transitions. All the theories use the same one-parameter ground-state Hylleraas' wave function [95]. The present computations in the CB1-4B (full black), BCIS-4B (full blue), BDW-4B (dashed blue) and CDW-4B (full black) methods are all for the order $N=192$ per integration axis in the Gauss-Legendre quadrature. The results of the CDW-EIS-4B method are from Ref. [55]. Experimental data: $\Delta$ [66], $\diamond$ [69], $\bigcirc$ [77], $\nabla$ [83], $\square$ [84], $\square$ [87] and $\bullet$ [88] (color online) 
$E=4000$ and $7000 \mathrm{keV}$. However, even this is inconclusive, since at e.g. $E=4000$ $\mathrm{keV}$, Schuch et al.'s experimental data [87] are by a factor of $\sim 20$ smaller than the measured findings from Afrosimov et al. [88]. New measurements would be highly desirable to help establish the high-energy behavior of total cross sections for double charge exchange in ion-atom collisions.

It is clear from Fig. 5 that the BCIS-4B method outperforms the CDW-4B method at $E<4000 \mathrm{keV}$. As was usual with $Q_{\mathrm{CDW}-3 \mathrm{~B}}$ for single charge exchange, $Q_{\mathrm{CDW}-4 \mathrm{~B}}$ for double charge exchange also keeps on rising with the descending $E$ at which $Q_{\mathrm{BCIS}-4 \mathrm{~B}}$ becomes clearly peaked. A better agreement of $Q_{\mathrm{BCIS}-4 \mathrm{~B}}$ with experimental data seen in Fig. 5 than in the case of $Q_{\mathrm{CDW}-4 \mathrm{~B}}$ points to a pronounced sensitivity of the cross section to the number of included continuum intermediate states (4 in the CDW-4B and 2 in the BCIS-4B method, as stated). Also the results for $Q_{\mathrm{CDW}-\mathrm{EIS}-4 \mathrm{~B}}$ are peaked around $100-200 \mathrm{keV}$, but these cross sections are orders of magnitudes smaller than $Q_{\text {BCIS-4B }}$.

It is noticeable that $Q_{\mathrm{CDW}-\mathrm{EIS}-4 \mathrm{~B}}$ hugely underestimates both $Q_{\mathrm{BCIS}-4 \mathrm{~B}}$ and experimental data at all impact energy. This is remarkable given that both $Q_{\mathrm{CDW}-\mathrm{EIS}-4 \mathrm{~B}}$ and $Q_{\text {BCIS-4B }}$ make use of the logarithmic Coulomb phases in one of the two scattering channels. However, the eikonalization in the BCIS-4B method is harmless as it relates to the relative motion of heavy nuclei. In contradistinction, however, the eikonalization from $Q_{\mathrm{CDW}-\text { EIS-4B }}$ refers to the electronic Coulomb wavefunctions and this turns out to be harmful. As mentioned, the differences between the Coulomb phases in the CDW-EIS-4B and BCIS-4B methods are inconsequential at infinitely large distances. However, charge exchange is a local process whose probability is negligible only for small electron-nucleus distances. This occurrence invalidates the replacement of the two full Coulomb wavefunctions by their asymptotic behaviors in the CDW-EIS-4B method.

Figure 6 is similar to Fig. 5 in every respect except that $Q_{\mathrm{BDW}-4 \mathrm{~B}}$ is plotted instead of $Q_{\mathrm{BCIS}-4 \mathrm{~B}}$. Here, the graphed $Q_{\mathrm{BDW}-4 \mathrm{~B}}$ also refers to the same two values of the Gauss-Legendre order, $N=48$ and 192 as was in Fig. 5. The overall discussion and conclusion reached for Fig. 5 applies to Fig. 6, as well. Next, Figs. 7 and 8 also refer separately to $N=48$ and 192 , respectively ${ }^{2}$. However, this time both $Q_{\mathrm{BCIS}-4 \mathrm{~B}}$ and $Q_{\mathrm{BDW}-4 \mathrm{~B}}$ are plotted together on each of these two figures so as to more clearly exhibit the similarities and differences between the BCIS-4B and BDW-4B methods on a larger scale than that from Fig. 4.

\section{Discussion and conclusion}

The present study deals with several well-established four-body distorted wave methods for two-electron capture by heavy nuclei from heliumlike atomic systems. The quantum-mechanical distorted wave formalism with all the four active particles is used with no recourse whatsoever to the semi-classical impact parameter method. As an illustration, we consider total cross sections for double charge-exchange in collisions

\footnotetext{
2 The case with $N=48$ has previously been condidered also in Refs. [8, 40]. In the caption to Fig. 1 of Ref. [8], the number of the Gauss-Legendre integration points per axis in the pertinent 5-dimensional integrals should have been stated to be 48 instead of 96 .
} 
of alpha particles with helium atomic targets at a wide impact energy range (100$10000 \mathrm{keV}$ ). Five methods (BCIS-4B, BDW-4B, CB1-4B, CDW-4B, CDW-EIS-4B) are compared with each other, as well as with the existing experimental data available at energies 100-7000 keV. The total cross sections $(Q)$ in the employed first-order (CB1-4B) and second-order (BCIS-4B, BDW-4B, CDW-4B, CDW-EIS-4B) methods for the general case of arbitrary nuclear charges necessitate multiple numerical integrations or quadratures.

For such collisions, three quadratures are required for computing $Q_{\mathrm{CB} 1-4 \mathrm{~B}}$. On the other hand, $Q_{\mathrm{CDW}-4 \mathrm{~B}}$ as well $Q_{\mathrm{CDW}-\mathrm{EIS}-4 \mathrm{~B}}$ invoke four numerical integrations. As to both $Q_{\mathrm{BCIS}-4 \mathrm{~B}}$ and $Q_{\mathrm{BDW}-4 \mathrm{~B}}$, five quadratures are needed. For the special case with symmetric encounters (e.g. alpha particles with helium atoms), only a single numerical integration ought to be performed in $Q_{\mathrm{CB} 1-4 \mathrm{~B}}$. Nevertheless, even for this particular case, the cross sections $Q_{\mathrm{CB} 1-4 \mathrm{~B}}$ have been generated by using the general program with any nuclear charges involving three-dimensional quadratures. This is done to bring closer the numerical effort invested in obtaining $Q_{\mathrm{CB} 1-4 \mathrm{~B}}$ to the more involved computations for $Q_{\mathrm{CDW}-4 \mathrm{~B}}, Q_{\mathrm{CDW}-\mathrm{EIS}-4 \mathrm{~B}}, Q_{\mathrm{BCIS}-4 \mathrm{~B}}$ and $Q_{\mathrm{BDW}-4 \mathrm{~B}}$. Moreover, for consistency, when comparing our results for $Q_{\mathrm{CB} 1-4 \mathrm{~B}}$ with $Q_{\mathrm{CDW}-4 \mathrm{~B}}, Q_{\mathrm{BCIS}-4 \mathrm{~B}}$ and $Q_{\mathrm{BDW}-4 \mathrm{~B}}$, the same Gauss-Legendre order $N$ is used per each integration axis in the CB1-4B, CDW-4B, BCIS-4B and BDW-4B methods (for each $N$, there are $N$ pivots and $N$ weights). The exception is the use of the Gauss-Mehler quadrature rule for the azimuthal angle $\phi_{\tau}$ from the numerical integration over momentum vector $\tau$ associated with the Fourier transform in the CDW-4B method. It was sufficient to use the Gauss-Mehler order $M=20$ because a thorough check computation yielded the same results for $Q_{\mathrm{CDW}-4 \mathrm{~B}}$ with $M=40$.

With this setting, the convergence rate of $Q_{\mathrm{CB} 1-4 \mathrm{~B}}, Q_{\mathrm{CDW}-4 \mathrm{~B}}, Q_{\mathrm{BCIS}-4 \mathrm{~B}}$ and $Q_{\mathrm{BDW}-4 \mathrm{~B}}$ for varying $N$ depends exclusively on the behavior of the integrands (the functions to be integrated). The results for $Q_{\mathrm{CDW}-\mathrm{EIS}-4 \mathrm{~B}}$ have not been computed in the present work and they are taken from Ref. [55]). We vary $N$ from 48 to 192 in steps of $16(N=48,64, \ldots, 192)$. The integrands encountered in $Q_{\mathrm{CB} 1-4 \mathrm{~B}}$ and $Q_{\mathrm{CDW}-4 \mathrm{~B}}$ are perfectly smooth and, consequently, these cross sections are found to converge remarkably fast, thus attaining the final results already with $N=48$ at nearly all impact energies. Due to use of the integral representations for the two Kummer confluent hypergeometric functions $\left({ }_{1} F_{1}\right)$, the integrands in $Q_{\mathrm{BCIS}-4 \mathrm{~B}}$ and $Q_{\mathrm{BDW}-4 \mathrm{~B}}$ contain the branch points at 0 and 1 . Nevertheless, after the simultaneous twofold Cauchy regularization at these two branch points, the integrands in BCIS-4B and BDW-4B methods become smooth. As a result, the convergence rate of $Q_{\mathrm{BCIS}-4 \mathrm{~B}}$ and $Q_{\mathrm{BDW}-4 \mathrm{~B}}$ is good with the ensuing accurate findings with the lowest order $N=48$ at most of the considered impact energies $150 \mathrm{keV} \leq E \leq 7000 \mathrm{keV}$.

The general transition amplitudes (for any initial and final states) in the three-body versions of the BCIS and BDW methods (i.e. BCIS-3B, BDW-3B) coincide with each other for one-electron capture by any nuclei from any hydrogenlike atomic targets. The ensuing results for $Q_{\mathrm{BCIS}-3 \mathrm{~B}}$ and $Q_{\mathrm{BDW}-3 \mathrm{~B}}$ are identical. Such an occurrence is due to the availability of the exact hydrogenlike wavefunctions. This ceases to be the case for two-electron capture by nuclei from heliumlike targets. The reason is in the unavailability of the exact heliumlike wavefunctions. In such cases, it is important to compare $Q_{\mathrm{BCIS}-4 \mathrm{~B}}$ and $Q_{\mathrm{BDW}-4 \mathrm{~B}}$ for double charge exchange in four-body rear- 
rangement collisions. For two-electron transfer from helium by alpha particles, it is reassuring to find that $Q_{\mathrm{BCIS}-4 \mathrm{~B}}$ and $Q_{\mathrm{BDW}-4 \mathrm{~B}}$ are in close mutual agreement. This happens even for the presently used simplest one-parameter Hylleraas' ground-state wavefunction of helium.

Ionization dominates over charge exchange at high energies for both one- and twoelectron transitions. This demands inclusion of ionization channels in the intermediate stage of the given capture collisional process. In other words, prior to being captured, electrons should first be ionized and then captured from continuum intermediate states. Such a situation requires a two-step process much in the line of the classical Thomas' double scattering, originally investigated for a purely three-body electron capture collision. It is this two-step mechanism which leads to the notion of second-order methods. There is also the one-step mechanism yielding the first-order methods. Here, electronic transitions are caused by direct collisions of projectile nuclei with target electrons. At high impact energies, the two-step mechanism dominates over the singlestep mechanism. This is evidenced already in a purely three-body capture process by inspecting the two sharply different asymptotic expressions of $Q$ at high energies in the first- and second-order methods.

The most striking difference between the first-and second-order estimates of $Q$ for three-body rearrangement collisions at very high energies occurs when either the initial or final bound states (or both) have non-zero angular momenta $\left(l_{i}, l_{f}\right)$. This is seen from the behaviors $Q_{\text {First-order }} \sim v^{-12-l_{i}-l_{f}}$ and $Q_{\text {Second-order }} \sim v^{-11}$ at asymptotically high values of the impact velocity $v$. Measured charge-exchange cross sections favor the asymptotic estimates from the second-order methods for zero- or non-zero angular momenta. This is also the case at intermediate-to-high (non-asymptotic) nonrelativistic energy region. The second-order is the lowest-order for which a three-body capture process can be described by pure classical mechanics. This is Thomas' billiardlike double collisions comprised of two steps or components. Here, a rearrangement collision is conceived as being built from two consecutive elastic collisions that result in the appearance of a peak near $\vartheta_{\text {lab }}=0.47 \mathrm{mrad}$ in differential cross sections. This Thomas peak dominates the forward peak at asymptotically high energies. A single step mechanism, as a first-order approximation, is feasible in quantum mechanics. It is made possible by the velocity matching resonance when a large electronic momentum in the final momentum-space hydrogenlike wavefunctions in the exit channel becomes close to the velocity of a scattered projectile. The resulting cross sections are, however, negligible (at asymptotically large impact energies) compared to the cross sections from the Thomas two-step mechanism.

The same or even a more pronounced situation with two Thomas peaks applies to double charge exchange. Two-electron capture is a much weaker process (less probable) than one-electron capture. This makes double capture more sensitive to the usual assumptions of the theory than in the case of single-electron capture. The most dramatic example is an assumption about the presumed validity of eikonalization of electronic continuum intermediate states when going from one- to two-electron capture. This assumption turned out to be reasonably adequate in the CDW-EIS-3B method for single charge-exchange, but fails flagrantly in the CDW-EIS-4B method for double charge-exchange. In particular, within the validity domain of high-energy theories, total cross sections in the CDW-EIS-4B method for the ground-to-ground-state tran- 
sition in double charge exchange in the $\alpha-\mathrm{He}$ collisions underestimates (by orders of magnitudes) the corresponding results from the CDW-4B method and experimental data.

At the same time, both the BCIS-4B and BDW-4B methods perform by far much better than the CDW-EIS-4B method. This is seen in good agreement between experimental data and BCIS-4B as well as BDW-4B methods at energies above $700 \mathrm{keV}$ for double charge exchange in the $\alpha-\mathrm{He}$ collisions. The BCIS-4B and BDW-4B methods also employ the eikonalized continuum intermediate states, but these are for relative motions of heavy nuclei. Such heavy-particle eikonalizations are adequate and the same total cross sections are obtained by using either the full Coulomb wavefunctions of nuclei or their logarithmic Coulomb phases. This hold true, not only in the BCIS or BDW methods, but also in the CDW and CB1 methods (or in any other method) for single as well as double charge exchange.

Acknowledgements Open access funding provided by Karolinska Institute. The author thanks two Research Funds: Radiumhemmet via the Karolinska University Hospital and the Stockholm County Council (FoUU).

Open Access This article is licensed under a Creative Commons Attribution 4.0 International License, which permits use, sharing, adaptation, distribution and reproduction in any medium or format, as long as you give appropriate credit to the original author(s) and the source, provide a link to the Creative Commons licence, and indicate if changes were made. The images or other third party material in this article are included in the article's Creative Commons licence, unless indicated otherwise in a credit line to the material. If material is not included in the article's Creative Commons licence and your intended use is not permitted by statutory regulation or exceeds the permitted use, you will need to obtain permission directly from the copyright holder. To view a copy of this licence, visit http://creativecommons.org/licenses/by/4.0/.

\section{References}

1. I.M. Cheshire, Continuum distorted wave approximation; resonant charge transfer by fast protons in atomic hydrogen. Proc. Phys. Soc. 84, 89-98 (1964)

2. Dž. Belkić, A quantum theory of ionization in fast collisions between atoms and ions. J. Phys. B 11, 3529-3552 (1978)

3. Dž. Belkić, R. Gayet, A. Salin, Electron capture in high-energy ion-atom collisions. Phys. Rep. 56, 279-369 (1979)

4. Dž. Belkić, Quantum Theory of High-Energy Ion-Atom Collisions (Taylor and Francis, London, 2008)

5. Dž. Belkić, I. Mančev, J. Hanssen, Four-body methods for high-energy ion-atom collisions. Rev. Mod. Phys. 80, 249-314 (2008)

6. Dž. Belkić, Review of theories on double electron capture in fast ion-atom collisions. J. Math. Chem. 47, 1420-1467 (2010)

7. V.Yu. Lazur, M.V. Khoma, Distorted wave theories for one- and two-electron capture in fast atomic collisions. Adv. Quantum Chem. 65, 363-405 (2013)

8. Dž. Belkić, Double charge exchange in ion-atom collisions using distorted wave theories with twoelectron continuum intermediate states in one or both scattering channels. J. Math. Chem. 57, 1-58 (2019)

9. Dž. Belkić, Theory of heavy ion collision physics in Hadron therapy (Ch 11), in State-of-the-Art Reviews on Energetic Ion-Atom and Ion-Molecule Collisions, ed. by Dž. Belkić, I. Bray, A. Kadyrov (World Scientific Publishing, Singapore, 2019), pp. 285-336

10. D.S.F. Crothers, J.F. McCann, Ionization of atoms by ion impact. J. Phys. B 16, 3229-3242 (1983)

11. J.M. Maidagan, R.D. Rivarola, A symmetric eikonal-type approximation for electron capture in ionatom collisions. J. Phys. B 17, 2477-2487 (1984)

12. V.I. Gerasimenko, L.N. Rosentsveig, Two-electron charge exchange of $\alpha$-particles in helium. J. Exp. Theor. Phys. JETP 4, 509-512 (1957) [Zh. Eksp. Teor. Fiz. 31, 684-687 (1956)] 
13. V.I. Gerasimenko, The two-electron charge exchange of protons in helium during fast collisions. J. Exp. Theor. Phys. JETP 14, 789-791 (1962) [Zh. Eksp. Teor. Fiz. 41, 1104-1106 (1961)]

14. M.J. Fulton, M.H. Mittleman, Electron capture by helium ions in neutral helium. Proc. Phys. Soc. 87, 669-676 (1966)

15. K. Roy, S.C. Mukherjee, D.P. Sural, $\mathrm{H}^{-}$formation in proton-helium collisions. Phys. Rev. A 13, 987-991 (1976)

16. S. Biswas, K. Bhadra, D. Basu, Double-electron capture by protons from helium. Phys. Rev. A 15, 1900-1905 (1977)

17. C.D. Lin, Double K-shell electron capture for ion-atom collisions at intermediate energies. Phys. Rev. A 19, 1510-1516 (1979)

18. C.D. Lin, P. Richard, Inner-shell vacancy production in ion-stom collisions. Adv. Atom. Mol. Phys. 17, 275-253 (1982)

19. R. Shingal, C.D. Lin, Calculations of two-electron transition cross sections between fully stripped ions and helium atoms. J. Phys. B 24, 251-264 (1991)

20. E.O. Alt, G.V. Avakov, L.D. Blokhintsev, A.S. Kadyrov, A. Mukhamedzhanov, Charge-exchange reactions ina three-body eikonal approach. J. Phys. B 27, 4653-4674 (1994)

21. C. Chaudhuri, S. Sanyal, T.K. Rai Dastidar, Use of a diabatic molecular expansion in studies on electron capture by $\mathrm{He}^{2+}$ ions in helium at keV energies. Pramana J. Phys. 43, 175-179 (1994)

22. C. Chaudhuri, S. Sanyal, T.K. Rai Dastidar, Theoretical study of single and double charge transfer in $\mathrm{He}^{2+}-\mathrm{He}$ collisions at kilo-electron-volt energies in a diabatic molecular representation. Phys. Rev. A 52, 1137-1142 (1995)

23. C. Chaudhuri, $\mathrm{He}^{2+}-\mathrm{He}$ charge transfer collisions using a 17-state close-coupling calculations with a diabatic molecular basis. PMC Phys. B (2009). https://doi.org/10.11861/1754-0429-2-2

24. T.C. Theisen, J.H. McGuire, Single and double electron capture in the independent-electron approximation at high velocities. Phys. Rev. A 20, 1406-1408 (1979)

25. R. Gayet, R.D. Rivarola, A. Salin, Double electron capture by fast nuclei. J. Phys. B 14, 2421-2427 (1981)

26. R. Gayet, Multiple capture and ionization in high-energy ion-atom collisions. J. Phys. (Paris), Colloque C1, Suppl. \# 1, 50, 53-71 (1989)

27. R. Gayet, J. Hanssen, A. Martínez, R. Rivarola, CDW and CDW-EIS investigations in an independent electron approximation for the resonant double electron capture by swift $\mathrm{He}^{2+}$ in He. Z. Phys. D 18, 345-350 (1991)

28. V.A. Sidorovich, V.S. Nikolaev, J.H. McGuire, Calculation of charge-changing cross sections in collisions of $\mathrm{H}^{+}, \mathrm{He}^{2+}$ and $\mathrm{Li}^{3+}$ with He atom. Phys. Rev. A 31, 2193-2201 (1985)

29. S.N. Chatterjee, B.N. Roy, Modified BEA calculations of $\mathrm{He}^{2+}$ impact double electron capture cross sections of atoms. J. Phys. B 18, 4283-4293 (1985)

30. M. Ghosh, C.R. Mandal, S.C. Mukherjee, Single and double electron capture from lithium by fast $\alpha$ particles. J. Phys. B 18, 3797-3803 (1985)

31. M. Ghosh, C.R. Mandal, S.C. Mukherjee, Double electron capture from helium by ions of helium, lithium, carbon and oxygen. Phys. Rev. A 35, 5259-3803 (1985)

32. R.E. Olson, A.E. Wetmore, M.L. McKenzie, Double electron transitions in collisions between multiply charged ions and helium atoms. J. Phys. B 19, L629-L634 (1986)

33. D.S.F. Crothers, R. McCarroll, Correlated continuum distorted-wave resonant double electron capture in $\mathrm{He}^{2+}-\mathrm{He}$ collisions. J. Phys. B 20, 2835-2842 (1987)

34. K.M. Dunseath, D.S.F. Crothers, Transfer and ionization processes during the collision of fast $\mathrm{H}^{+}, \mathrm{He}^{2+}$ nuclei with helium. J. Phys. B 20, 5003-5022 (1991)

35. M. Kimura, Single and double electron capture in $\mathrm{He}^{2+}+\mathrm{He}$ collisions and single electron capture in $\mathrm{He}^{+}+\mathrm{He}^{+}$collisions. J. Phys. B 21, L19-L24 (1988)

36. G. Deco, N. Grün, An approximate description of the double capture process in $\mathrm{He}^{2+}+\mathrm{He}$ collisions with static correlation. Z. Phys. D 18, 339-343 (1991)

37. M.S. Gravielle, J.E. Miraglia, Double-electron capture as a two-step process. Phys. Rev. A 45, 2965-2973 (1992)

38. Dž. Belkić, I. Mančev, Formation of $\mathrm{H}^{-}$by double charge exchange in fast proton-helium collisions. Phys. Scr. 45, 35-42 (1992)

39. Dž. Belkić, I. Mančev, Four-body CDW approximation: dependence of prior and post total cross sections for double charge exchange upon bound-state wave-functions. Phys. Scr. 46, 18-23 (1993) 
40. Dž. Belkić, Importance of intermediate ionization continua for double charge exchange at high energies. Phys. Rev. A 47, 3824-3844 (1993)

41. Dž. Belkić, Symmetric double charge exchange in fast collisions of bare nuclei with helium-like atomic systems. Phys. Rev. A 47, 189-200 (1993)

42. Dž. Belkić, Two-electron capture from helium-like atomic systems by completely stripped projectiles. J. Phys. B 26, 497-508 (1993)

43. L. Gulyás, Gy Szabo, Resonant double electron capture by fast $\mathrm{He}^{2+}$ from helium: the first-order Born approximation with correct boundary condition. Z. Phys. D 29, 115-119 (1994)

44. E. Ghanbari-Adivi, Coulomb-Born distorted wave approximation applied to the proton-helium singleelectron capture process. J. Phys. B 44, 165204 (2011)

45. E. Ghanbari-Adivi, H. Ghavaminia, Electron capture by alpha particles from helium atoms in a Coulomb-Born distorted-wave approximation. J. Phys. B 45, 235202 (2012)

46. E. Ghanbari-Adivi, A.N. Velayati, K-shell single-electron capture in collision of fast protons with multi-electron atoms. Cent. Eur. J. Phys. 11, 423-430 (2013)

47. E. Ghanbari-Adivi, H. Ghavaminia, Double-electron capture in collision of fast alpha particles with helium atoms. Int. J. Mod. Phys. 23, 1450079 (2014)

48. H. Ghavaminia, E. Ghanbari-Adivi, Influence of electron correlations on double-capture process in proton-helium collisions. Chin. Phys. B 24, 073401 (2015)

49. Dž. Belkić, Double charge exchange at high impact energies. Nucl. Inst. Methods Phys. Res. B 86, 62-81 (1994)

50. Dž. Belkić, I. Mančev, M. Mudrinić, Two-electron capture from helium by fast alpha particles. Phys. Rev. A 49, 3646-3658 (1994)

51. R. Gayet, J. Hanssen, A. Martínez, R. Rivarola, Double electron capture in ion-atom collisions at high impact velocities. Nucl. Instr. Methods Phys. Res. B 86, 158-160 (1994)

52. R. Gayet, J. Hanssen, A. Martínez, R. Rivarola, Status of two-electron processes in ion-atom collisions at intermediate and high energies. Comments Atom. Mol. Phys. 30, 231-248 (1994)

53. R. Gayet, J. Hanssen, L. Jacqui, A. Martínez, R. Rivarola, Double electron capture by fast bare ions in helium atoms: production of singly and doubly excited states. Phys. Scr. 53, 549-556 (1996)

54. A.E. Martínez, H.F. Busnengo, R. Gayet, J. Hanssen, R.D. Rivarola, Double electron capture in atomic collisions at intermediate and high collision energies: contribution of capture into excited states. Nucl. Instr. Methods Phys. Res. B 132, 344-349 (1997)

55. A.E. Martínez, R.D. Rivarola, R. Gayet, J. Hanssen, Double electron capture theories: second order contributions. Phys. Scr. T80, 124-127 (1999)

56. A.L. Ford, L.A. Wehrman, K.A. Hall, J.F. Reading, Single and double electron removal from helium by protons. J. Phys. B 30, 2889-2897 (1997)

57. M. Purkait, Double electron capture cross-sections of the ground state in the collisions of $\mathrm{He}^{2+}$ and $\mathrm{Li}^{3+}$ with He. Eur. Phys. J. D 30, 11-14 (2004)

58. M. Purkait, S. Sounda, A. Dhara, C.R. Mandal, Double charge transfer cross sections in inelastic collisions of bare ions with helium atoms. Phys. Rev. A 74, 042723 (2006)

59. S. Ghosh, A. Dhara, C.R. Mandal, M. Purkait, Double electron capture cross sections from helium by fully stripped projectile ions in intermediate-to-high energies. Phys. Rev. A 78, 042708 (2008)

60. S. Ghosh, A. Dhara, C.R. Mandal, M. Purkait, Two electron capture cross sections into ground state in collision of bare projectile ions with helium atoms. Fizika A 18, 9-18 (2009)

61. S. Ghosh, A. Dhara, M. Purkait, C.R. Mandal, Double charge transfer cross sections in collisions of protons with helium. Indian J. Phys. 83, 231-243 (2010)

62. S. Samaddar, S. Halder, A. Mondal, C.R. Mandal, M. Purkait, T.K. Das, Single and double electron capture in $p-$ He and $\alpha-$ He collisions. J. Phys. B 50, 065202 (2017)

63. S.K. Alisson, Experimental results on charge-changing collisions of hydrogen and helium atoms and ions above $0.2 \mathrm{keV}$. Rev. Mod. Phys. 30, 1137-1168 (1958)

64. Ya M. Fogel', R.V. Mitin, V.F. Kozlov, N.D. Romashko, On applicability of the Massey adiabatic hypothesis to double charge exchange. J. Exp. Theor. Phys. JETP 8, 390-398 (1959) [Zh. Eksp. Teor. Fiz. 35, 565-573 (1958)]

65. V.S. Nikolaev, L.N. Fateeva, I.S. Dmitriev, Ya.A. Teplova, Capture of several electrons by fast multicharged ions. J. Exp. Theor. Phys. JETP 14, 67-74 (1962) [Zh. Eksp. Teor. Fiz. 41, 89-99 (1961)]

66. L.I. Pivovar, M.T. Novikov, V.M. Tubaev, Electron capture by helium ions in various gases in the 300-1500 keV energy range. J. Exp. Theor. Phys. JETP 15, 1035-1039 (1962) [Zh. Eksp. Teor. Fiz. 42, 1490-1494 (1962)] 
67. J.F. Williams, Cross sections for double electron capture by $2-50 \mathrm{keV}$ protons incident upon hydrogen and the inert gases. Phys. Rev. A 150, 7-10 (1966)

68. U. Schryber, Elektroneneinfang schneller protonen in gasen. Helv. Phys. Acta 40, 1023-1051 (1967)

69. K.H. Berkner, R.V. Pyle, J.W. Stearns, J.C. Warren, Single- and double-electron capture by 7.2 to 181 $\mathrm{keV}^{3} \mathrm{He}^{++}$ions in He. Phys. Rev. 166, 44-46 (1968)

70. L.H. Toburen, M.Y. Nakai, Double electron capture cross sections for incident protons in the energy range 75 to $250 \mathrm{keV}$. Phys. Rev. 177, 191-196 (1969)

71. M.B. Shah, H.B. Gilbody, Formation of $\mathrm{He}^{+}(2 s)$ metastable ions in passage of $10-60 \mathrm{keV}^{3} \mathrm{He}^{2+}$ ions through gases. J. Phys. B 7, 256-268 (1974)

72. M.B. Shah, H.B. Gilbody, Single and double ionisation of helium by $\mathrm{H}^{+}, \mathrm{He}^{2+}$ and $\mathrm{Li}^{3+}$ ions. J. Phys. B 18, 899-913 (1985)

73. J.E. Bayfield, G.A. Khayrallah, Electron transfer in keV-energy ${ }^{4} \mathrm{He}^{++}$atomic collisions. I. Single and double electron transfer with $\mathrm{He}, \mathrm{Ar}, \mathrm{H}_{2}$ and $\mathrm{N}_{2}$. Phys. Rev. A 11, 920-929 (1975)

74. J.E. Bayfield, G.A. Khayrallah, Electron transfer in keV-energy ${ }^{4} \mathrm{He}^{++}$collisions. II. Formation of ${ }^{4} \mathrm{He}^{++}-\mathrm{He}^{+}(2 s)$ in collisions with $\mathrm{He}, \mathrm{Ar}, \mathrm{H}_{2}$ and $\mathrm{N}_{2}$. Phys. Rev. A 11, 930-941 (1975)

75. H. Schrey, B. Huber, Total cross section measurements for charge exchange of $\mathrm{He}^{++}$ions with $\mathrm{He}$ and Ar atoms. Z. Phys. A 273, 401-403 (1975)

76. C.W. Woods, R.L. Kauffman, K.A. Jamison, N. Stolterfoht, P. Richard, K-shell Auger-electron hypersatellites of Ne. Phys. Rev. A 12, 1393-1398 (1975)

77. E.W. McDaniel, M.R. Flannery, H.W. Ellis, F.L. Eisele, W. Pope, US Army Missile Research and Development Command, Technical Report, No. H-78-1 (1977)

78. A. Itoh, M. Asari, F. Fukuzawa, Charge-changing collisions of $0.7-2.0 \mathrm{MeV}$ helium beams in various gases. I. Electron capture. J. Phys. Soc. Jpn. 48, 943-950 (1980)

79. I.S. Dmitriev, N.F. Vorob'ev, Zh.M. Konovalova, V.S. Nikolaev, V.N. Novozhilova, Ya.A. Teplova, Yu.A. Faïnberg, Loss and capture of electrons by fast ions and atoms of helium in various media. J. Exp. Theor. Phys. JETP 57, 1157-1164 (1983) [Zh Eksp, Teor. Fiz. 84, 1987-2000 (1983)]

80. M.E. Rudd, T.V. Goffe, A. Itoh, Ionization cross sections for 10-300-keV/u and electron-capture cross sections for 5-150-keV/u ${ }^{3} \mathrm{He}^{2+}$ ions in gases. Phys. Rev. A 32, 2128-2133 (1985)

81. M. Sasao, K. Sato, A. Matsumoto, A. Nishizawa, S. Takagi, S. Amemiya, T. Masuda, Y. Tsurita, F. Fukuzawa, Y. Haruyama, Y. Kanamori, Electron capture cross sections in high energy $\mathrm{He}^{2+}+\mathrm{Li}$ collisions. J. Phys. Soc. Jpn. 55, 102-105 (1986)

82. R. Hippler, S. Datz, P. Miller, P. Pepmiler, P. Dittner, Double and single electron capture and loss in collisions of 1-2 MeV/u boron, oxygen, and silicon projectiles with helium atoms. Phys. Rev. A 35, 585-590 (1987)

83. R.D. DuBois, Ionization and charge transfer in $\mathrm{He}^{2+}-$ rare gas collisions: II. Phys. Rev. A 36, 2585-2593 (1987)

84. N.V. de Castro Faria, F.L. Freire Jr., A.G. de Pinho, Electron loss and capture by fast helium ions in noble gases. Phys. Rev. A 37, 280-283 (1988)

85. W.K. Wu, B.A. Huber, K. Wiesemann, Cross sections for electron capture by neutral and charged particles in collisions with He. Atom. Data Nucl. Data Tables 42, 157-186 (1989)

86. J.H. Posthumus, P. Lukey, R. Morgenstern, Double electron capture into highly charged ions: correlated or independent? Z. Phys. D Suppl. 21, S285-S286 (1991)

87. R. Schuch, E. Justiniano, H. Vogt, G. Deco, N. Grün, Double electron capture by $\mathrm{He}^{2+}$ from He at high velocity. J. Phys. B 24, L133-L138 (1991)

88. V.V. Afrosimov, D.F. Barash, A.A. Basalaev, N.A. Guschchina, K.O. Lozhkin, V.K. Nikulin, M.N. Panov, IYu. Stepanov, Single- and double-electron capture from many-electron atoms by $\alpha$ particles in the MeV energy range. J. Exp. Theor. Phys. JETP 77, 554-561 (1993) [Zh. Eksp. Teor. Fiz. 104, 3297-3310 (1993)]

89. R. Dörner, V. Mergel, L. Spielberger, O. Jagutzki, H. Schmidt-Böcking, J. Ullrich, State-selective differential cross sections for double-electron capture in $0.25-0.75 \mathrm{MeV} \mathrm{He}^{2+}-\mathrm{He}$ collisions. Phys. Rev. A 57, 312-317 (1998)

90. H. Bräuning, H. Helm, J.S. Briggs, E. Salzborn, Double electron transfer in $\mathrm{H}^{-}+\mathrm{H}^{+}$collisions. J. Phys. Conf. Ser. 88, 012033 (2007)

91. M. Schulz, T. Vajnai, J.A. Brand, Differential double capture cross sections in $p-$ He collisions. Phys. Rev. A 75, 022717 (2007) 
92. M.M. Sant'Anna, A.C.F. Santos, L.S.F. Coelho, G. Jalbert, N.V. de Castro Faria, F. Zappa, P. Focke, Dž. Belkić, Double electron capture cross sections for $\mathrm{Li}^{3+}$ collisions with $\mathrm{He}$ at intermediate-to-high velocities. Phys. Rev. A 80, 042707 (2009)

93. N.V. Novikov, Ya.A. Teplova, Database on charge-changing cross sections in ion-atom collisions. J. Phys. Conf. Ser. 194, 082032 (2009)

94. I.S. Dmitriev, Ya.A. Teplova, Yu.A. Belkova, N.V. Novikov, Yu.A. Faïnberg, Experimental electron loss and capture cross sections in ion-atom collisions. Atom. Data Nucl. Data Tables 96, 85-121 (2010)

95. E.A. Hylleraas, Neue Berechtnung der Energie des Heliums im Grundzustande, sowie tiefsten Terms von Ortho-Helium. Z. Phys. 54, 347-366 (1929)

96. Dž. Belkić, Electron capture by fast protons from helium, nitrogen and oxygen: the corrected first Born approximation. Phys. Rev. A 37, 55-67 (1988)

97. C.J. Joachain, R. Vanderpoorten, Configuration-interaction wave functions for two-electron systems. Physica 46, 333-343 (1970)

Publisher's Note Springer Nature remains neutral with regard to jurisdictional claims in published maps and institutional affiliations. 